CERN-TH-2011-133

MCNET-11-19

\title{
Timelike Dipole-Antenna Showers with Massive Fermions
}

\author{
A. Gehrmann-De Ridder ${ }^{1}$, M. Ritzmann ${ }^{1}$, P. Skands ${ }^{2}$ \\ 1: Institute for Theoretical Physics, ETH, CH-8093 Zurich, Switzerland \\ 2: Theoretical Physics, CERN, CH-1211 Geneva 23, Switzerland
}

\begin{abstract}
We present a complete formalism for final-state (timelike) dipole-antenna showers including fermion masses, but neglecting polarization and finite-width effects. We make several comparisons of tree-level expansions of this shower algorithm to fixed-order matrix elements for hadronic $Z$ decays, up to and including $Z \rightarrow 6$ partons, to which the algorithm can be consistently matched over all of phase space. We also compare to analytical resummations at the NLL level. The shower algorithm has been implemented in the publicly available VINCIA plugin to the PYTHIA 8 event generator, which enables us to compare to experimental data at the fully hadronized level. We therefore also include comparisons to selected observables in $b$-tagged $Z$ decays.
\end{abstract}

\section{Introduction}

The large phase space opened up by the LHC is rekindling interest in the collider phenomenology of heavy coloured particles. Appreciable samples of top quarks with large Lorentz boosts are becoming accessible for the first time; energetic top and bottom quarks are sought as decay products of newphysics or Higgs particles; and coloured new-physics particles may also themselves give off radiation, though at suppressed rates close to threshold.

Indeed, for massive particles produced near threshold, most of the radiation produced results from the violent deceleration of the incoming (massless) colour charges, i.e initial state radiation is dominant. The effects of multiple soft emissions from the massive partons themselves are then largely unimportant for the description of the event as a whole and only become relevant to define precisely the mass of the produced particle [1-3].

However, for the production of boosted heavy quarks or other coloured particles, either directly, via decay, or through gluon splitting processes, multiple emissions cannot be neglected. Mass corrections will generate differences in the shape of the evolving jet, and in the energy loss of the evolving particle. These effects must be taken systematically into account if we are to rely on physics models of these phenomena to distinguish "signal" from "background" production sources.

On the theory side, calculations of observables involving massive particles present a unique set of challenges. The introduction of an additional scale in the problem, for each non-zero mass, leads to an increased number of terms in amplitudes, to modifications to the pole structure caused by the massive propagators and to more complicated phase-space boundaries and kinematics. The presence of massive final state particles shrinks the size of the phase space available for additional QCD emissions, both in fixed-order calculations and in parton showers. 
The modifications to the pole structure imply different infrared limits in the massive case. In particular, QCD radiation from massive particles can lead to soft divergences but cannot lead to strict collinear divergences, since the mass is acting as an infrared regulator. Traditional Monte Carlo (MC) shower descriptions, which rely on the relative dominance of collinear-enhanced terms, therefore become intrinsically less accurate when non-zero masses are involved. Though it is possible to systematically improve shower descriptions to take into account universal mass effects (as, e.g., in [4]), one would still expect a relatively larger uncertainty from non-universal and/or subleading terms than in the massless case, simply because the leading singular behaviour itself is less strong. Consequently, corrections from higher-order matrix elements, generally referred to as "matrix element matching", may be relatively more important.

In this paper, we shall attempt to address a relevant subset of these challenges, in the specific context of matched time-like dipole-antenna showers [5,6]. We restrict our attention to unpolarized stable massive particles, deferring a detailed treatment of helicity dependence, as discussed recently by [7] and finite-width effects to a future study. Still, our approach has some advantages. To the best of our knowledge, this is the first time a rigorous and systematic approach to mass effects has been incorporated in an antenna-based shower Monte Carlo code [5, 8,9]. We also generalize the fixed-order antenna functions derived in [10-14] to include variations in their non-singular behaviour and extend the unitarity-based matching formalism presented in [6] to include tree-level matrix elements with up to four additional massive partons beyond the Born level. Due to the unitary nature of the matching corrections, this prescription can be used also in the soft and (quasi-)collinear regions and hence we expect the subleading properties of the resulting shower to be improved. This is a feature which is not possible with other approaches to multileg matching, such as MENLOPS [15], CKKW [16, 17] and related approaches [18-20], or MLM (see [21] for a description). The speed of the resulting matched calculations is also greatly improved as compared to the existing approaches, as discussed in [22].

Corrections at the next-to-leading order level have not yet been included in this work. We therefore do not attempt to distinguish rigorously between different possible mass definitions, such as "constituent" vs "pole" vs "running" masses [2,3,23]. For the purpose of our studies here, we treat parton masses simply as effective parameters, to be determined from data. It has been argued that this should be comparable to using a perturbative mass definition evaluated at a scale of the order of the infrared shower cutoff [24], though the corresponding scheme is only defined numerically by the shower algorithm. We expect that NLO matching for massive fermions will be able to provide some further insight into this question, but that is beyond the scope of the work presented here.

This paper is organized as follows. In section 2, we discuss the factorization, kinematics, and infrared limits of a single $2 \rightarrow 3$ splitting involving massive partons. In section 3, we introduce the additional ingredients required to turn this into a framework for parton showering, including a discussion of trial functions and veto algorithm steps. The generalization of our evolution variables to the massive case and the treatment of $g \rightarrow q \bar{q}$ splittings in the shower are also addressed. Sections 4, 5, and 6 then present comparisons to fixed-order matrix elements, to analytical resummations, and to $b$-tagged experimental data, respectively. We round off with conclusions and an outlook in section 7.

We note that the work reported has been made publicly available as a plug-in to the PYTHIA 8 event generator [25], starting from VINCIA version 1.026 [26]. 


\section{Massive Phase-Space Factorization and Massive Dipole-Antennae}

The dipole-antenna formalism [12,27-29] is constructed from two basic ingredients:

1) an exact momentum-conserving and Lorentz-invariant phase-space factorization based on $2 \rightarrow 3$ mappings between on-shell partons

2) a set of antenna functions that, combined with the phase-space factorization, capture the leading singular behaviour of gauge field theory amplitudes [28].

We return to how these are implemented in the shower context in section 3. In this section, we focus on a single "elementary" $2 \rightarrow 3$ branching, e.g., as it would appear during a single step in a shower algorithm, and/or in the context of an antenna-based NLO calculation. We here focus on the generalizations necessary in the massive case, with details on the massless treatment available in [5, 6, 12]. We begin by giving some conventions concerning the notations we use, in section 2.1, then turn to the phase-space factorization in sections 2.2-2.3 and finally discuss the structure of the antenna functions in sections 2.4-2.5.

\subsection{Notation and conventions}

Given momenta $p_{a}, p_{b}$ of massive particles $a$ and $b$ with masses $m_{a}$ and $m_{b}$, it is convenient to use the notation,

$$
s_{a b}=2 p_{a} \cdot p_{b}=\left(p_{a}+p_{b}\right)^{2}-m_{a}^{2}-m_{b}^{2},
$$

which we adopt throughout this paper. With this notation, the relation expressing the conservation of the total centre-of-mass (CM) energy in a massive $2 \rightarrow 3$ branching, $I K \rightarrow i j k$, becomes

$$
m_{I K}^{2}=\left(p_{I}+p_{K}\right)^{2}=s_{I K}+m_{I}^{2}+m_{K}^{2}=s_{i j}+s_{j k}+s_{i k}+m_{i}^{2}+m_{j}^{2}+m_{k}^{2} .
$$

The momenta involved in this branching are either called parent or pre-branching momenta for $I, K$ and daughter or post-branching momenta for $i, j, k$. Hence we may express, the dot product of two daughter momenta $i, k$ as, $s_{i k}=m_{I K}^{2}-s_{i j}-s_{j k}-m_{i}^{2}-m_{j}^{2}-m_{k}^{2}$.

We shall also work with scaled invariants, normalized to the CM energy of the dipole-antenna given by $m_{I K}^{2}$

and scaled mass values,

$$
y_{a b}=\frac{s_{a b}}{m_{I K}^{2}},
$$

$$
\mu_{a}=\frac{m_{a}}{m_{I K}} .
$$

We denote dipole-antenna functions by the symbol $a$ (for antenna) and represent the partons participating in the branching process $I K \rightarrow i j k$ by subscripts $a_{j / I K}$. The normalization of $a$ is such that $\left|M_{n+1}\right|^{2} \sim a\left|M_{n}\right|^{2}$ in the relevant soft/collinear limits, to be elaborated upon in the sections below. We also define a corresponding colour- and coupling-stripped dipole-antenna function $\bar{a}_{j / I K}$. The relation between $a$ and $\bar{a}$ is ${ }^{1}$

$$
a_{j / I K}=\frac{\alpha_{s}}{4 \pi} \mathcal{C}_{j / I K} \bar{a}_{j / I K}
$$

\footnotetext{
${ }^{1}$ Note that compared to the equivalent relation between these two antenna functions presented in [6], the normalization to the phase-space factor is not present here anymore. We prefer to keep the phase-space factor normalization outside the dipole-antenna functions which are defined from matrix elements squared only.
} 
where $\mathcal{C}_{j / I K}$ denotes the appropriate colour factor for the branching, as follows: $\widehat{C}_{F}$ for $q \bar{q} \rightarrow q g \bar{q}, C_{A}$ for $g g \rightarrow g g g$, either $\widehat{C}_{F}$ or $C_{A}$ for $q g \rightarrow q g g$, and $\widehat{T}_{R}$ for the splitting of a gluon into a quark-antiquark pair. In the conventions for the colour factors we use [6], we have $C_{A}=N_{C}, \widehat{C}_{F}=2 C_{F}=N_{C}-1 / N_{C}$, $\widehat{T}_{R}=2 T_{R}=1$ which makes the difference between $\widehat{C}_{F}$ and $C_{A}$ explicitly colour-subleading.

Note furthermore that the lower index of the dipole-antenna function $a_{j / I K}$ fully specifies the partons involved in the branching process, and hence those functions are uniquely determined. In particular, the radiators $I K$ are also uniquely identified, and our antenna functions are therefore related to so-called "sub-antenna" functions in the context of fixed-order subtraction [12].

\subsection{Phase-space factorization}

To define the dipole-antenna phase space characterizing the massive $2 \rightarrow 3$ branching process $I, K \rightarrow$ $i, j, k$, we consider the exact factorization of the $n+1$-particle phase space $\mathrm{d} \Phi_{n+1}$ into a $n$-particle phase space $\mathrm{d} \Phi_{n}$ and a dipole-antenna phase space given by $\mathrm{d} \Phi_{3} / \mathrm{d} \Phi_{2}$

$$
\mathrm{d} \Phi_{n+1}\left(p_{1} \ldots, p_{i}, p_{j}, p_{k}, \ldots p_{n+1} ; q\right)=\mathrm{d} \Phi_{n}\left(p_{1} \ldots, p_{I}, p_{K}, \ldots p_{n+1} ; q\right) \frac{\mathrm{d} \Phi_{3}\left(p_{i}, p_{j}, p_{k}\right)}{\mathrm{d} \Phi_{2}\left(p_{I}, p_{K}\right)}
$$

In this equation, $\mathrm{d} \Phi_{n}$ corresponds to the phase space for $n$ outgoing particles with momenta $p_{1}, . . p_{n}$ and masses $m_{1} . . m_{n}$, with total four-momentum $q^{\mu}$. In $\mathrm{d} \Phi_{n}$ only the parent momenta $p_{I}, p_{K}$ appear. The relation between $p_{I}, p_{K}$ and $p_{i}, p_{j}, p_{k}$, typically called "momentum mapping" in fixed-order subtraction contexts and "recoil strategy" in parton-shower ones, will be discussed in section 2.3 below.

The dipole antenna phase space $\frac{\mathrm{d} \Phi_{3}{ }^{i j k}}{\mathrm{~d} \Phi_{2} I K}$ is proportional to the three-particle phase space. It involves only the pre- and post-branching momenta $p_{I}, p_{K}$ and $p_{i}, p_{j}, p_{k}$ respectively and is given by $[5,6,13]$,

$$
\frac{\mathrm{d} \Phi_{3}{ }^{i j k}}{\mathrm{~d} \Phi_{2}{ }^{I K}}=\frac{1}{16 \pi^{2}} \frac{1}{\sqrt{\lambda\left(m_{I K}^{2}, m_{I}^{2}, m_{K}^{2}\right)}} \mathrm{d} s_{i j} \mathrm{~d} s_{j k} \frac{\mathrm{d} \phi}{2 \pi},
$$

where the Källen function $\lambda$ is given by

$$
\lambda(a, b, c)=a^{2}+b^{2}+c^{2}-2(a b+b c+a c),
$$

$\phi$ parametrizes rotations around the $\mathbf{P}_{I}-\mathbf{P}_{K}$-axis in the centre-of-mass frame. As long as we restrict ourselves to unpolarized processes which we do in this paper, all emission probabilities are independent of $\phi$. The factor $\mathrm{d} \phi /(2 \pi)$ will therefore be suppressed in the following.

The boundaries of the three-particle phase space with general masses follow from momentum conservation and the on-shell conditions. They are given by

$$
\begin{gathered}
2 m_{i} m_{j}=s_{i j}^{-} \leq s_{i j} \leq s_{i j}^{+}=\left(m_{I K}-m_{k}\right)^{2}-m_{i}^{2}-m_{j}^{2} \\
s_{j k}^{ \pm}\left(s_{i j}\right)=\frac{1}{2\left(s_{i j}+m_{i}^{2}+m_{j}^{2}\right)}\left[\left(s_{i j}+2 m_{j}^{2}\right)\left(\left[s_{i j}^{+}+2 m_{k}\left(m_{I K}-m_{k}\right)\right]-s_{i j}\right)\right. \\
\left. \pm \sqrt{s_{i j}^{2}-\left(s_{i j}^{-}\right)^{2}} \sqrt{s_{i j}^{+}-s_{i j}} \sqrt{s_{i j}^{+}+4 m_{I K} m_{k}-s_{i j}}\right] .
\end{gathered}
$$


Equivalently, these boundaries characterizing the physical phase space for the daughter partons $i, j, k$ are determined by requiring the positivity of the Gram determinant $\Delta_{3}$ defined as,

$$
\Delta_{3}=\frac{1}{4}\left(s_{i j} s_{i k} s_{j k}-s_{i j}^{2} m_{k}^{2}-s_{i k}^{2} m_{j}^{2}-s_{j k}^{2} m_{i}^{2}+4 m_{i}^{2} m_{j}^{2} m_{k}^{2}\right)
$$

\subsection{Phase-space mappings}

To specify the phase-space factorization in equation (6), a momentum-conserving mapping, or "recoil strategy" in the parton shower language, that relates the three on-shell daughter momenta, $p_{i}, p_{j}$, and $p_{k}$ to the two on-shell parent momenta $p_{I}$ and $p_{K}$ is needed. In a dipole-antenna approach, the radiators $I$ and $K$ can be both emitter or recoiler and the radiation emitted between them is shared smoothly and symmetrically amongst them. The mapping between daughter and parent momenta presented below will reflect this fundamental property. Independent of whether the momenta involved are massive or not, the on-shell and momentum-conserving $2 \rightarrow 3$ mapping is not unique except on the boundaries of the phase space. Instead, there is a one-parameter family of such mappings.

Generalizing the analysis in [28] to the case of non-vanishing particle masses we start by relating the momenta of the daughter particles to those of the parent momenta as follows,

$$
\begin{aligned}
p_{I} & =x p_{i}+r p_{j}+z p_{k} \\
p_{K} & =(1-x) p_{i}+(1-r) p_{j}+(1-z) p_{k}
\end{aligned}
$$

with the on-shell conditions

$$
p_{i}^{2}=m_{i}^{2} \quad, \quad p_{j}^{2}=m_{j}^{2} \quad, \quad p_{k}^{2}=m_{k}^{2} \quad, \quad p_{K}^{2}=m_{K}^{2} \quad, \quad p_{I}^{2}=m_{I}^{2}
$$

We use these on-shell conditions to re-express the parameters $x$ and $z$ in terms of the single free parameter $r$ and obtain

$$
\begin{aligned}
x=\frac{1}{2\left(4 \Delta_{3}+m_{I K}^{2}\left(s_{i k}^{2}-\left(s_{i k}^{-}\right)^{2}\right)\right)}\left[\Sigma^{2}\left(s_{i k}^{2}-\left(s_{i k}^{-}\right)^{2}+4 \Delta_{i j}\right)\right. \\
\left.+R\left(s_{i j}^{+}+2 m_{I K} m_{k}-s_{i j}\right)+8 r\left(\Delta_{3}-m_{I K}^{2} \Delta_{i j}\right)\right] \\
z=\frac{1}{2\left(4 \Delta_{3}+m_{I K}^{2}\left(s_{i k}^{2}-\left(s_{i k}^{-}\right)^{2}\right)\right)}\left[\Sigma^{2}\left(s_{i k}^{2}-\left(s_{i k}^{-}\right)^{2}+4 \Delta_{j k}\right)\right. \\
\left.-R\left(s_{j k}^{+}+2 m_{I K} m_{i}-s_{j k}\right)+8 r\left(\Delta_{3}-m_{I K}^{2} \Delta_{j k}\right)\right]
\end{aligned}
$$


where we have defined

$$
\begin{aligned}
R^{2} & =16 \Delta_{3}\left[m_{I K}^{2} r(1-r)-(1-r) m_{I}^{2}-r m_{K}^{2}\right]+\left[s_{i k}^{2}-\left(s_{i k}^{-}\right)^{2}\right]\left[s_{I K}^{2}-\left(s_{I K}^{-}\right)^{2}\right] \\
s_{I K}^{-} & =2 m_{I} m_{K} \\
\Sigma^{2} & =m_{I K}^{2}+m_{I}^{2}-m_{K}^{2} \\
\Delta_{i j} & =(-1) \cdot \operatorname{det}\left(\begin{array}{cc}
\frac{s_{i j}}{2} & \frac{s_{j k}}{2} \\
\frac{s_{i k}}{2} & m_{k}^{2}
\end{array}\right)=\frac{1}{4}\left(s_{j k} s_{i k}-2 s_{i j} m_{k}^{2}\right) \\
\Delta_{j k} & =(-1) \cdot \operatorname{det}\left(\begin{array}{cc}
m_{i}^{2} & \frac{s_{i j}}{2} \\
\frac{s_{i k}}{2} & \frac{s_{j k}}{2}
\end{array}\right)=\frac{1}{4}\left(s_{i j} s_{i k}-2 s_{j k} m_{i}^{2}\right) .
\end{aligned}
$$

These equations characterize our 1-parameter family of massive mappings. The parameters $z$ and $x$ are related to each other with the replacements $i \leftrightarrow k$ and $R \rightarrow-R$. Contrary to the massless case, however, $R^{2}>0$, (which corresponds to real momentum fractions $x$ and $z$ ), is not true for arbitrary values of the momentum fraction $r$.

We note that the massive dipole mapping of [30] corresponding to a dipole made of the partons $i, j$ and $k$ which play respectively the roles of emitter $(i)$, emittee $(j)$ and spectator $(k)$, is obtained as a special case, by setting $r=x$ in the above formula. In this case we have,

$$
x=\frac{\Sigma^{2}}{2 m_{I K}^{2}}+\frac{\left(s_{i j}^{+}+2 m_{I K} m_{k}-s_{i j}\right) \sqrt{s_{I K}^{2}-\left(s_{I K}^{-}\right)^{2}}}{2 m_{I K}^{2} \sqrt{\lambda\left(m_{I K}^{2},\left(p_{i}+p_{j}\right)^{2}, m_{k}^{2}\right)}}
$$

where, in the rest frame of $p_{i}+p_{j}$, we can rewrite the Källén function using

$$
\lambda\left(m_{I K}^{2},\left(p_{i}+p_{j}\right)^{2}, m_{k}^{2}\right)=4\left(p_{i}+p_{j}\right)^{2} E_{k}^{2} \mathbf{v}_{k}^{2}
$$

where $E_{k}$ denotes the energy of the parton $k, \mathbf{v}_{k}$ its three-velocity. As in the massless case, the massive dipole mapping is asymmetric under the interchange of the particles $i$ and $k$, but symmetric under the interchange of $i$ and $j$. While it is appropriate to use this mapping for a shower based on CSdipoles (CS:Catani-Seymour) which distinguishes between emitter $i$, emittee $j$ and spectator $k$, it would clearly be inappropriate to use it in a dipole-antenna shower like VINCIA where the roles of $i$ and $k$ are interchangeable. The dipole mapping is mentioned for comparison only.

To get a geometrical picture of the mapping used in VINCIA, it is convenient to express the free parameter $r$ in the massive mapping family presented above in terms of the angle $\psi$ between the daughter parton $p_{i}$ and the parent parton $p_{I}$ (see, e.g., [5,8]). To this end, we write down the 4-product $p_{i} \cdot p_{I}$ as:

$$
\begin{aligned}
p_{i} \cdot p_{I}= & E_{i} E_{I}-\left|p_{i}\right|\left|p_{I}\right| \cos \psi \\
= & \frac{1}{4\left(4 \Delta_{3}+m_{I K}^{2}\left(s_{i k}^{2}-\left(s_{i k}^{-}\right)^{2}\right)\right.}\left[\Sigma^{2}\left(s_{i k}^{2}-\left(s_{i k}^{-}\right)^{2}\right)\left(s_{j k}^{+}+2 m_{I K} m_{i}-s_{j k}\right)\right. \\
& \left.\quad+8 r\left(s_{j k}^{+}+2 m_{I K} m_{i}-s_{j k}\right) \Delta_{3}-R\left(s_{i k}^{2}-\left(s_{i k}^{-}\right)^{2}+4 \Delta_{j k}\right)\right] .
\end{aligned}
$$


Looking at equations (17) and equations (24), we see that if we approach the boundaries of the phase space (for example if we consider a soft emission $p_{j} \rightarrow 0$ or if we take the (quasi-)collinear limit for $p_{i}$ and $p_{j}$ ), the Gram-determinant $\Delta_{3}$ tends to zero and the dependence of $p_{i} \cdot p_{I}$ on the free parameter $r$ drops out. ${ }^{2}$

Inside the parton shower VINCIA, to define appropriately the $2 \rightarrow 3$ branching, we need to fix the mapping. In other words, we need to fix the functional form of the free parameter $r$. If all particles are massless, the default mapping used is given by [28]

$$
r=\frac{s_{j k}}{s_{i j}+s_{j k}} .
$$

This mapping has the properties that the interchange $i \leftrightarrow k$ corresponds to $r \leftrightarrow(1-r)$ and the momentum fractions are restricted to $x \geq 1,0 \leq r \leq 1$ and $z \leq 0$, where $r=0$ corresponds to the collinear limit $p_{j} \| p_{k}$ and $r=1$ corresponds to the collinear limit $p_{i} \| p_{j}$.

In the massive case, we shall consider the following mapping,

$$
\begin{aligned}
r & =r^{-}+\frac{s_{j k}-s_{j k}^{-}}{s_{i j}-s_{i j}^{-}+s_{j k}-s_{j k}^{-}}\left(r^{+}-r^{-}\right) \\
& =\frac{\Sigma^{2}}{2 m_{I K}^{2}}+\frac{\sqrt{s_{I K}^{2}-\left(s_{I K}^{-}\right)^{2}}}{2 m_{I K}^{2}} \frac{s_{j k}-s_{j k}^{-}-\left(s_{i j}-s_{i j}^{-}\right)}{s_{i j}-s_{i j}^{-}+s_{j k}-s_{j k}^{-}}
\end{aligned}
$$

where the condition

$$
r^{-}=\frac{\Sigma^{2}-\sqrt{s_{I K}^{2}-\left(s_{I K}^{-}\right)^{2}}}{2 m_{I K}^{2}} \leq r \leq r^{+}=\frac{\Sigma^{2}+\sqrt{s_{I K}^{2}-\left(s_{I K}^{-}\right)^{2}}}{2 m_{I K}^{2}}
$$

ensures that $R^{2}>0$.

Apart from reducing to the massless mapping in equation (25) for vanishing masses, this phase-space mapping has the "swapping" property that $i \leftrightarrow k$ combined with $I \leftrightarrow K$ corresponds to $r \leftrightarrow(1-r)$. For $m_{I}=m_{i}$ and $m_{K}=m_{k}$ it also satisfies $x \geq 1$ and $z \leq 0$ and can therefore be viewed as a generalization of the massless mapping given in equation (25).

In figure 1, the mapping given in equation (26) is illustrated in a Dalitz plot of the three-particle phase space of the daughter momenta $p_{i}, p_{j}, p_{k}$. The phase-space boundary is marked with a solid grey line. Insets show the orientation of the daughter momenta for a branching with $s_{i j}$ and $s_{j k}$ given by the centre of the inset, in the $\mathrm{CM}$ frame of the parent partons, with $\phi$ is chosen such that the radiated particle is moving "upwards". A mass configuration characteristic for $Q \bar{q} \rightarrow Q g \bar{q}$, with $m_{Q}=0.25 m_{I K}$, $m_{\bar{q}}=0$ (left), is compared to the massless case (right). Notice that the physically allowed phase space shrinks considerably in the massive case, and that the invariant $s_{i j}$ can only vanish in the soft limit $p_{j} \rightarrow 0$. The limit $s_{i j} \rightarrow 0$ with $j$ hard is not accessible. The mass effects on the mapping are most pronounced for configurations which are close to the edge of the phase space and far away from the soft limit. For the rest of the phase space they are relatively unimportant. A similar illustration for massless partons can be found in [6].

\footnotetext{
${ }^{2}$ The sole exception to this occurs for $s_{i k}=s_{i k}^{-}$where the angle $\psi$ does depend on the functional form of $r$.
} 


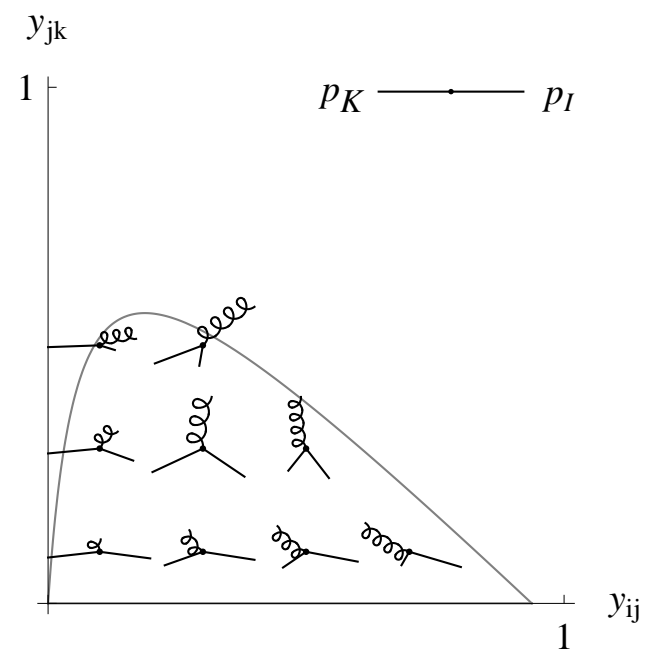

(a) $Q \bar{q} \rightarrow Q g \bar{q}(Q$ massive, $\bar{q}$ massless $)$

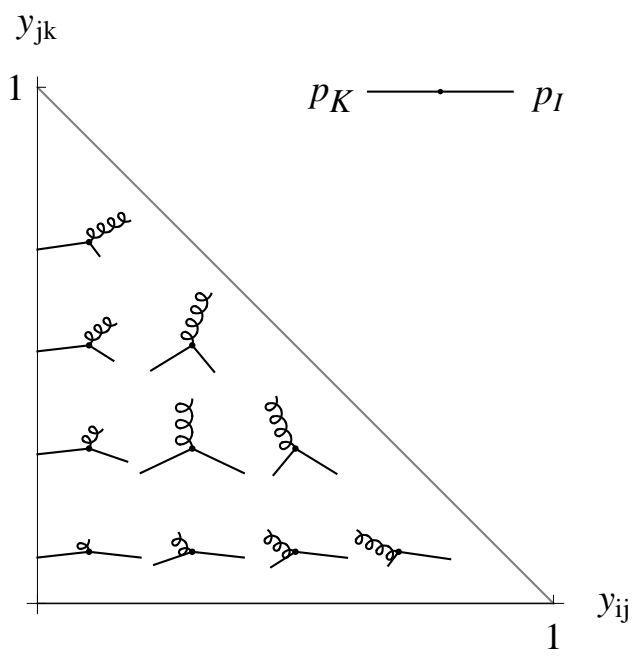

(b) $q \bar{q} \rightarrow q g \bar{q}$ ( $q$ massless $)$

Figure 1: Dalitz plot of the dipole-antenna phase space for $I K \rightarrow i j k$ for massive partons (left) as compared to massless ones (right), using the scaled invariants $y_{a b}$ defined in equation (3) as coordinates. The boundary of the physically allowed phase space is drawn as a solid grey line. Insets show the orientation of the $i j k$ momenta corresponding to the centre of each inset, in the CM frame of the parent partons, with parents oriented horizontally and $\phi$ chosen such that the gluon is radiated "upwards". The mass values used in the left-hand pane are $m_{I}=$ $m_{i}=0.25 m_{I K}, m_{K}=m_{k}=m_{j}=0$.

\subsection{Pole structure}

Since masses act as infrared regulators in the collinear region, the pole structure of massive amplitudes is actually simpler (less divergent) than that of their massless counterparts. A specific example of this is given in figure 2, in which we show the ratio of the amplitudes squared for the processes $Z \rightarrow Q g \bar{Q}$ relative to $Z \rightarrow q g \bar{q}$, as a function of the $Q g$ opening angle, for $M_{Z}=91 \mathrm{GeV}, E_{g}=10 \mathrm{GeV}$ and $m_{Q}=4.8 \mathrm{GeV}$ ( $Q$ stands for a massive quark while $q$ stands for a massless one). The dip in the thick solid line for $\theta_{i j} \rightarrow 0$ is generated by the mass-shielding of the collinear enhancements, relative to the massless case (thin line).

However, the calculation of observables with massive final state particles still involves the treatment of potentially large mass-dependent logarithmic terms. They correspond to collinear divergences which are regulated by the quark mass, therefore they become divergent in the massless limit. For observables that are infrared safe in the massless limit, these logarithmic terms cancel in the final result, but they can still appear at intermediate steps of the calculation, for example in the separate evaluation of real and virtual contributions. They are of the form $\ln \left(Q^{2} / m^{2}\right)$, where $m$ is the parton mass and $Q$ is a characteristic scale of the hard-scattering process. These mass-dependent logarithmic terms are related to the quasi-collinear [31] limit of the matrix element, the definition of which we shall recall below.

In a fixed-order approach, the potentially large logarithmic contributions induced by mass terms are taken care of in the context of subtraction methods [32]; terms which mimic the singular behaviour of real matrix elements are added and subtracted. The construction of these terms relies heavily on the factorization properties of amplitudes in their soft and (quasi-)collinear limits [31]. In the antenna 


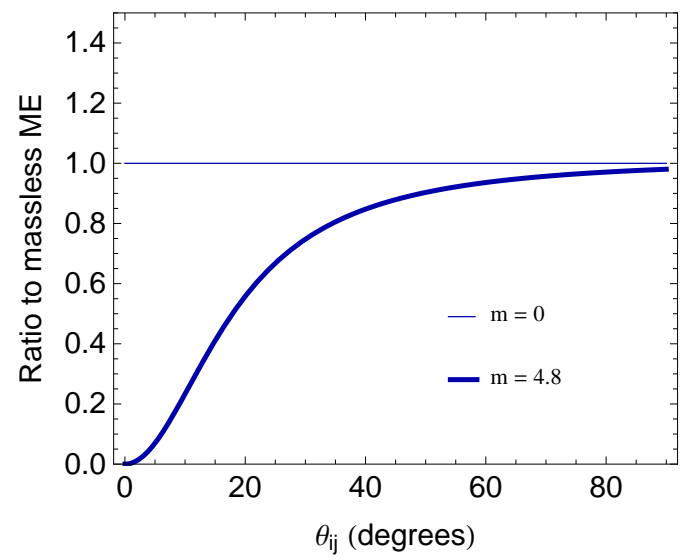

Figure 2: Illustration of the dampening of the collinear singularity for $Z \rightarrow Q g \bar{Q}$ : squared matrix elements with (thick) and without (thin) mass corrections, normalized to the massless case, as a function of the opening angle between the quark and the gluon, for constant $E_{g}=10 \mathrm{GeV}$ and $m_{Q}=4.8 \mathrm{GeV}$.

framework presented in [13,14] (and in the dipole formalism [30] that predates it), the main building blocks, massive antenna (dipole) functions and phase-space factorizations, are therefore constructed so as to reproduce exactly the quasi-collinear and soft behaviours of real radiation matrix-elements in the corresponding limits. For cross sections which are well-behaved in the massless limit, the explicit cancellations of the $\ln \left(Q^{2} / m^{2}\right)$-terms also ensure numerical stability in the limit $m \rightarrow 0$.

For some observables which are not infrared safe in their massless limit, such as ones sensitive to the details of the fragmentation process for example, the cancellation of the mass-dependent logarithms is incomplete. Terms of the form $\alpha_{S}^{n} \ln ^{n}\left(Q^{2} / m^{2}\right)$ appear in every order of the expansion. In the case of a large hierarchy $m \ll Q$, these terms jeopardize the convergence of the perturbative series. It is necessary to resum them to all orders to obtain a meaningful result, as is done, for example, for the $b$ quark fragmentation process in [33], to which we compare the massive VINCIA dipole-antenna shower in section 5. However, in order to construct this shower, we must first consider the soft and quasicollinear limits more carefully and define how the massless splitting functions and soft Eikonal factors are generalized in the presence of massive particles.

The infrared singularity properties of tree-level colour-ordered matrix elements involving only massless partons have been well studied in [31]. In the limit where a gluon $j$ is soft with respect to its neighbouring partons $i$ and $k$, the colour-ordered matrix-elements squared $\left|\mathcal{M}_{n+1}\right|^{2}$ for $(n+1)$ partons factorizes into a universal soft Eikonal factor $S_{i j k}$ and a colour-ordered tree-level squared amplitude where gluon $j$ has been removed. For the squared amplitudes we have,

$$
\left|\mathcal{M}_{n+1}(1, \cdots, i, j, k, \cdots, n+1)\right|^{2} \stackrel{j_{g} \rightarrow 0}{\longrightarrow} g_{s}^{2} \mathcal{C}_{i j k} S_{i j k}\left|\mathcal{M}_{n}(1, \cdots, i, k, \cdots, n+1)\right|^{2}
$$

where $g_{s}^{2}=4 \pi \alpha_{s}$ is the strong coupling, $\mathcal{C}_{i j k}$ is a colour factor that tends to $N_{C}$ in the leading-colour limit, and the massless Eikonal factor is given by

$$
S_{i j k}=\frac{2 s_{i k}}{s_{i j} s_{j k}} .
$$

Similarly when two neighbouring gluons or a quark and a gluon become collinear the colour-ordered matrix elements factorize. Depending on the nature of the partons involved different collinear factors 
are obtained. Partons which are not colour-connected do not lead to singular behaviours of the colour ordered matrix-elements squared, hence the soft or collinear factors only involve the neighbouring particles to which the unresolved particle is colour-connected.

In the massive case, essentially the same factorization properties still hold, provided the collinear limit is generalized to the quasi-collinear limit (see below). For the emission of a soft gluon from massive radiators, the factorization of the matrix element into a soft Eikonal factor times a reduced matrix element with the soft gluon omitted works in the same way as for massless partons. The soft Eikonal factor given in equation (29) needs however to be generalized. Written in terms of the parent parton masses $m_{I}$ and $m_{K}$ and the invariants between the daughter partons $i, j$ and $k$, the massive soft Eikonal factor reads

$$
S_{i j k}\left(m_{I}, m_{K}\right)=\frac{2 s_{i k}}{s_{i j} s_{j k}}-\frac{2 m_{I}^{2}}{s_{i j}^{2}}-\frac{2 m_{K}^{2}}{s_{j k}^{2}}
$$

which has two new mass-dependent terms compared to the massless Eikonal factor defined above.

The quasi-collinear limit of a massive parton with momentum $p^{\mu}$ decaying into two massive partons $j$ and $k$ is given by,

$$
\begin{gathered}
p_{j}^{\mu} \rightarrow z p^{\mu}, p_{k}^{\mu} \rightarrow(1-z) p^{\mu}, \\
p^{2}=m_{(j k)}^{2} .
\end{gathered}
$$

with the constraints,

$$
p_{j} \cdot p_{k}, m_{j}, m_{k}, m_{j k} \rightarrow 0
$$

at fixed ratios,

$$
\frac{m_{j}^{2}}{p_{j} \cdot p_{k}}, \frac{m_{k}^{2}}{p_{j} \cdot p_{k}}, \frac{m_{j k}^{2}}{p_{j} \cdot p_{k}} .
$$

The key difference between the massless collinear limit and the quasi-collinear limit is given by the constraint that the on-shell masses squared have to be kept of the same order as the invariant mass $\left(p_{j}+p_{k}\right)^{2}$, with the latter becoming small. In these corresponding quasi-collinear limits, the colourordered $(m+1)$-parton matrix element squared factorizes into a reduced $m$-parton matrix element squared multiplied by quasi-collinear splitting functions, the latter are generalizations of the AltarelliParisi splitting functions [34] from which they differ by mass-dependent terms. In four dimensions, they read

$$
\begin{aligned}
& P_{q g \rightarrow Q}\left(z, m_{q}, s_{q g}\right)=\frac{1+(1-z)^{2}}{z}-\frac{2 m_{q}^{2}}{s_{q g}}, \\
& P_{q \bar{q} \rightarrow G}\left(z, m_{q}, s_{q \bar{q}}\right)=z^{2}+(1-z)^{2}+\frac{2 m_{q}^{2}}{s_{q \bar{q}}+2 m_{q}^{2}} .
\end{aligned}
$$

We now turn to a description of the full massive dipole-antenna functions as implemented in VINCIA.

\subsection{Massive dipole-antenna functions}

In general, the full forms of the dipole-antenna functions are obtained by normalizing a three-parton tree-level matrix-element squared to a corresponding two-parton squared matrix element, stripped of all 
couplings and colour factors and normalized to reproduce the known collinear splitting functions and soft Eikonal factors in the corresponding unresolved limits.

In the fixed-order context, specific sets of such dipole-antenna functions have been derived for the massless case in [10-12] and for the massive one in [13,14]. In principle, there is an infinite set of similar dipole-antenna functions, differing by non-singular ("finite") terms and hence having the same soft and (quasi-)collinear limits, which could equally well be used to construct the subtraction terms. For example, consider gluon emission off a $q \bar{q}$ antenna. To cover the limiting behaviour of this emission process, we could use the matrix element for $\gamma^{*} \rightarrow Q g \bar{Q}$ normalized to the one for $\gamma^{*} \rightarrow Q \bar{Q}$,

$$
\bar{a}_{g / q \bar{q}}^{\gamma^{*} \rightarrow Q g \bar{Q}}\left(m_{I K}^{2}, s_{i j}, s_{j k}, m_{q}, m_{\bar{q}}\right)=\frac{2 s_{i k}}{s_{i j} s_{j k}}-\frac{2 m_{q}^{2}}{s_{i j}^{2}}-\frac{2 m_{\bar{q}}^{2}}{s_{j k}^{2}}+\frac{1}{s_{I K}+4 m_{q} m_{\bar{q}}}\left(\frac{s_{i j}}{s_{j k}}+\frac{s_{j k}}{s_{i j}}\right) .
$$

Alternatively, we could use the process $H \rightarrow Q g \bar{Q}$, which gives

$$
\bar{a}_{g / q \bar{q}}^{H \rightarrow Q g \bar{Q}}\left(m_{I K}^{2}, s_{i j}, s_{j k}, m_{q}, m_{\bar{q}}\right)=\frac{2 s_{i k}}{s_{i j} s_{j k}}-\frac{2 m_{q}^{2}}{s_{i j}^{2}}-\frac{2 m_{\bar{q}}^{2}}{s_{j k}^{2}}+\frac{1}{s_{I K}-2 m_{q} m_{\bar{q}}}\left(\frac{s_{i j}}{s_{j k}}+\frac{s_{j k}}{s_{i j}}+2\right) .
$$

In both of these expressions, the denominator factor $s_{I K}+x m_{q} m_{\bar{q}}$ is proportional to the two-parton matrix element to which the three-particle matrix element is normalized. These two different constructions would give rise to slightly different integrated and unintegrated subtraction terms, but the final result would in either case be completely independent of which one is used.

For a parton shower, however, the behaviour of the dipole-antenna functions away from the phasespace boundaries is important to determine the amount of radiation produced. The most obvious example is that of adding a positive constant to a dipole-antenna function. This would result in a slightly higher rate for hard emissions in the parton shower (and consequently smaller Sudakov factors) without changing the limiting behaviour of the dipole-antenna function. In the context of shower uncertainty evaluations, it is therefore useful to generalize the definition of the dipole-antennae, to allow for continuous variations of the ambiguous non-singular terms, as done for the massless case in [6]. The possibility of varying finite parts in the parton-shower framework is a particular and important feature of the VINCIA code. Other parton showers, whose evolution equations are based on fixed kernels, such as the Altarelli-Parisi splitting functions [34] and/or the Catani-Seymour dipole ones [35], do not provide this particular uncertainty measure.

However, the presence of quark masses greatly increases the number of possible finite terms that could be added, hence we shall still place some limitations on the type of terms we will allow for, as will be described in detail below. As a starting point, we require that we must be able to reproduce the dipole-antenna functions which were derived from physical matrix elements, such as those given above. We then choose a generalization of the resulting parametrization, in such a way that the finite parts of all the dipole-antenna functions are parametrized in a similar way. We consider it preferable to have a rather general parametrization of the finite parts of the antenna functions because a change in the parametrization itself would require a change in the program code, whereas changes to individual terms within a given parametrization can be made without even recompiling the code.

Subsequently, we must decide which values to assign the finite coefficients by default. Since we chiefly intend to use them for variations, our philosophy is to set most of them to zero from the start, allowing only for a few non-zero values to bring the tree-level expansion of the resulting parton shower 
into reasonable agreement with the fixed-order matrix elements for $Z$ decay up to $Z \rightarrow 6$ partons. Note that explicit comparisons to such matrix elements are given in section 4 .

In the context of our shower model, one must also require that the dipole-antenna functions be positive definite, since they act as branching probability densities. This is the case for all dipole-antennae considered in this paper.

With the default choices fixed, we also define two antenna-function variations which we consider reasonably extremal, which we call "MIN" and "MAX". Our approach here has been to choose the coefficients for the MIN set as small as possible without introducing negative values for the dipole-antennae and then choosing the MAX coefficients such that the difference between the default coefficients and the MIN coefficients is at least as big as that between the MAX coefficients and the default coefficients. Note that we have not varied all possible finite coefficients in the MIN and MAX sets, but only a small subset of them, so it is conceivable that some physical variations could fall outside the range we define here. As always, uncertainty evaluations are more of an art than an exact science. We expect to learn more about the reasonableness of our choices as we expand to more processes in the future and can make explicit comparisons to more matrix elements.

In the following we shall present the decomposition of the dipole-antenna functions used in VINCIA into their singular and finite parts. The following antennae are needed: gluon emission from a $q \bar{q}, q g$, and $g g$ parent antenna, and gluon splitting from a $q g$ or $g g$ parent. As mentioned in section 2.1, the hard radiators are always uniquely determined, and hence the antennae we discuss here are equivalent to the ones referred to as "sub-antennae" in fixed-order contexts (see, e.g., [12]). For each of these five antenna types, we give four separate sets of "finite terms": DEF (default), MIN, MAX, and GGG, with the latter reproducing the fixed-order antennae defined in [12-14]. Condensed summaries of the corresponding finite-term values are given in tables 1 , for gluon emission antennae, and in tables 2 , for gluon-splitting ones.

To define the dipole-antenna for gluon emission off a massive $Q \bar{Q}$ pair (where the quark and the antiquark may or may not be of identical flavour), we start with the generic form

$$
\begin{aligned}
& \bar{a}_{g / q \bar{q}}\left(m_{I K}^{2}, s_{i j}, s_{j k}, m_{q}, m_{\bar{q}}\right)= \\
& \quad \frac{1}{m_{I K}^{2}}\left(\frac{2 y_{i k}}{y_{i j} y_{j k}}-\frac{2 \mu_{q}^{2}}{y_{i j}^{2}}-\frac{2 \mu_{\bar{q}}^{2}}{y_{j k}^{2}}+\frac{1}{1-\mu_{q}^{2}-\mu_{\bar{q}}^{2}+x \mu_{q} \mu_{\bar{q}}}\left(\frac{y_{i j}}{y_{j k}}+\frac{y_{j k}}{y_{i j}}+F_{g / q \bar{q}}\right)\right),
\end{aligned}
$$

where $F_{g / q \bar{q}}$ represents an arbitrary "finite" function, i.e. a function which is regular in all soft and (quasi-)collinear limits. The antenna function derived from $Z$ decay, as in the fixed order context and called $A_{3}^{0}=a_{3}^{0}$ corresponds to $F_{g / q \bar{q}}=0$ with $x=4$ and $m_{q}=m_{\bar{q}}$ as listed in table 1 .

We allow for the following optional terms in $F_{g / q \bar{q}}$,

$$
\begin{aligned}
F_{g / q \bar{q}}\left(y_{i j}, y_{j k}, \mu_{q}, \mu_{\bar{q}}\right)=C_{00} & +C_{10}\left(y_{i j}+y_{j k}\right)+C_{20}\left(y_{i j}^{2}+y_{j k}^{2}\right)+C_{11} y_{i j} y_{j k} \\
& +\left(\mu_{q}+\mu_{\bar{q}}\right)\left(M_{00}^{10}+M_{10}^{10}\left(y_{i j}+y_{j k}\right)\right) \\
& +\left(\mu_{q}^{2}+\mu_{\bar{q}}^{2}\right)\left(M_{00}^{20}+M_{10}^{20}\left(y_{i j}+y_{j k}\right)\right) \\
& +\mu_{q} \mu_{\bar{q}}\left(M_{00}^{11}+M_{10}^{11}\left(y_{i j}+y_{j k}\right)\right)
\end{aligned}
$$

with the default values $C_{a b}=M_{a b}^{c d}=0$. Note that this form of $F$ explicitly respects charge conjugation symmetry $(i \leftrightarrow k)$. In principle, one could allow for terms with higher powers of masses and/or in- 
$\mathrm{q} \overline{\mathbf{q}} \rightarrow \mathrm{qg} \overline{\mathbf{q}}$

\begin{tabular}{lcccc}
\hline $\bar{a}_{g / q \bar{q}}$ & Def & GGG & MIN & MAX \\
\hline $\mathcal{C}$ & $\frac{8}{3}$ & $\frac{8}{3}$ & $\frac{8}{3}$ & $\frac{8}{3}$ \\
\hline$C_{00}$ & - & - & -6 & 6 \\
$C_{10}$ & - & - & 4.5 & -4.5 \\
$C_{01}$ & - & - & 4.5 & -4.5 \\
\hline$x$ & - & 4 & 4 & -2 \\
$M_{00}^{20}$ & - & - & 9 & - \\
\hline
\end{tabular}

gg $\rightarrow$ ggg

\begin{tabular}{lcccc}
\hline $\bar{a}_{g / q \bar{q}}$ & Def & GGG & MIN & MAX \\
\hline $\mathcal{C}$ & 3 & 3 & $\frac{8}{3}$ & 3 \\
\hline$C_{00}$ & 2 & $\frac{8}{3}$ & -8 & 10 \\
$C_{10}$ & - & -1 & 7.5 & -7.5 \\
$C_{01}$ & - & -1 & 7.5 & -7.5 \\
\hline
\end{tabular}

qg $\rightarrow$ qgg

\begin{tabular}{lcccc}
\hline $\bar{a}_{g / q \bar{q}}$ & Def & GGG & MIN & MAX \\
\hline $\mathcal{C}$ & 3 & 3 & $\frac{8}{3}$ & 3 \\
\hline$C_{00}$ & 2 & 2.5 & -6 & 10 \\
$C_{10}$ & -1 & -2 & 6 & -8
\end{tabular}

\begin{tabular}{lllll}
$C_{01}$ & - & -0.5 & 7 & -7 \\
\hline
\end{tabular}

$\begin{array}{lllll}M_{00}^{1} & - & 1 & - & -\end{array}$

$\begin{array}{lllll}M_{00}^{2} & - & -3.5 & 8.5 & -\end{array}$

$M_{10}^{2} \quad-\quad-\quad-8 \quad-$

$\begin{array}{lllll}M_{01}^{2} & - & - & 8 & -\end{array}$

$\begin{array}{lllll}M_{-11}^{2} & - & -2 & - & -\end{array}$

$\begin{array}{lllll}M_{-11}^{2} & - & -2 & - & - \\ \widetilde{M}_{-1-12}^{2} & - & -1 & - & -\end{array}$

$\begin{array}{lllll}\widetilde{M}_{-1-11}^{3} & - & -1 & - & -\end{array}$

$\begin{array}{cccc}\widetilde{M}_{-1-11}^{3} & - & -1 & - \\ \widetilde{M}_{-1-11}^{4} & - & 1 & -\end{array}$

Table 1: Colour factors and finite parts for four different examples of the colour-ordered gluon-emission antenna functions. The VINCIA default antenna set (Def) is compared to the "GGG", MIN, and MAX variations. Coefficients which are not listed (or which are represented by "-") are zero.

variants, but for the simple purpose of uncertainty estimates, we believe the form above gives sufficient flexibility. ${ }^{3}$

The default $Q \bar{Q}$ antenna function used in VINCIA is thus,

$$
\bar{a}_{g / q \bar{q}}^{(\mathrm{def})}\left(m_{I K}^{2}, s_{i j}, s_{j k}, m_{q}, m_{\bar{q}}\right)=\frac{1}{m_{I K}^{2}}\left(\frac{2 y_{i k}}{y_{i j} y_{j k}}-\frac{2 \mu_{q}^{2}}{y_{i j}^{2}}-\frac{2 \mu_{\bar{q}}^{2}}{y_{j k}^{2}}+\frac{1}{1-\mu_{q}^{2}-\mu_{\bar{q}}^{2}}\left(\frac{y_{i j}}{y_{j k}}+\frac{y_{j k}}{y_{i j}}\right)\right)
$$

which corresponds to choosing zero values for the finite part and for $x$ in equation (38). In the left-hand pane of table 1, we compare the default values in VINCIA to the GGG functions and to a "MIN" and "MAX" variation that we use for uncertainty estimates. Note that a non-zero $M$ coefficient is introduced in the "MIN" case, in order to avoid negative regions in the massive dipole-antenna function.

The influence of quark masses on the default $Q \bar{Q}$ antenna function is illustrated in figure 3. This figure shows contours of constant values for the antenna $a_{g / q \bar{q}}$ in a Dalitz plot of the three-particle phase space for massless quarks (short dashed) and for massive quarks (dashed). For massive quarks, the contour lines start to avoid the boundaries of the phase space (drawn as a solid grey line) for high values of the antenna function. This is a direct consequence of the presence of terms of the form $\left(-s_{i j}^{2} / m^{2}\right)$ in the massive soft Eikonal factor given in equation (30).

\footnotetext{
${ }^{3}$ The denominator factor $s_{I K}+x m_{q} m_{\bar{q}}$ in equation (38) has $x=0$ in VINCIA, but we retain the possibility to vary it for uncertainty estimates.
} 


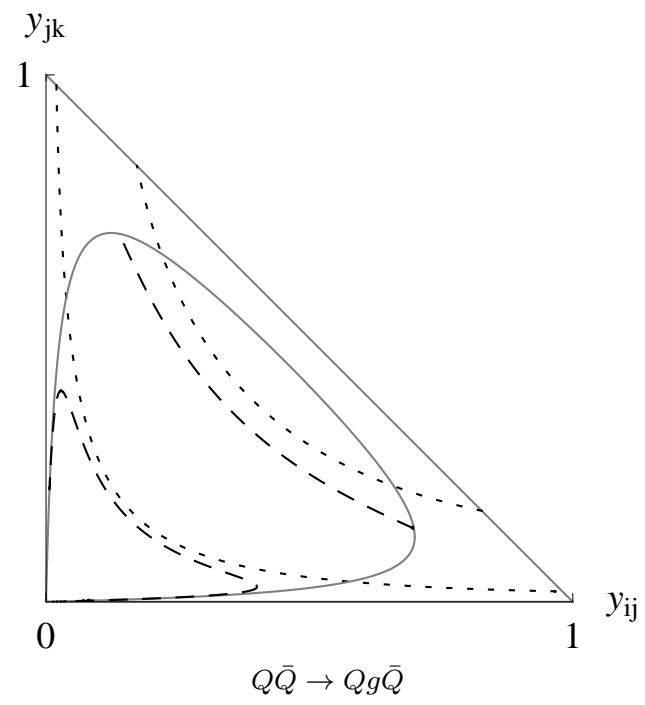

Figure 3: Dalitz plot showing contours of the massive (dashed) and massless (short dashed) gluon-emission dipole-antenna function $\bar{a}_{g / q \bar{q}}$, with $m_{Q}=0.15 m_{I K}$. Contours of constant values of the dipole-antennae are shown for $\bar{a}=5,50$. Grey solid lines denote the boundaries of the massless and of the massive phase space respectively.

For gluon emission in a $Q g$ dipole-antenna, we use the generic ${ }^{4}$ form

$$
\begin{aligned}
\bar{a}_{g / q g}\left(m_{I K}^{2}, s_{i j}, s_{j k}, m_{q}\right) & = \\
& \frac{1}{m_{I K}^{2}}\left(\frac{2 y_{i k}}{y_{i j} y_{j k}}-\frac{2 \mu_{q}^{2}}{y_{i j}^{2}}+\frac{1}{1-\mu_{q}^{2}}\left(\frac{y_{j k}}{y_{i j}}+\frac{y_{i j}}{y_{j k}}\left(1-\frac{y_{i j}}{1-\mu_{q}^{2}}\right)+\frac{1}{1-\mu_{q}^{2}} F_{g / q g}\right)\right) .
\end{aligned}
$$

We allow for the following finite terms,

$$
\begin{aligned}
F_{g / q g}=C_{00} & +C_{10} y_{i j}+C_{01} y_{j k}+C_{20} y_{i j}^{2}+C_{02} y_{j k}^{2}+C_{11} y_{i j} y_{j k} \\
& +\mu_{q}\left(M_{00}^{1}+M_{10}^{1} y_{i j}+M_{01}^{1} y_{j k}\right) \\
& +\mu_{q}^{2}\left(M_{00}^{2}+M_{10}^{2} y_{i j}+M_{01}^{2} y_{j k}+M_{-10}^{2} \frac{1}{y_{i j}}+M_{-11}^{2} \frac{y_{j k}}{y_{i j}}\right. \\
& \left.+\widetilde{M}_{-1-12}^{2} \frac{y_{j k}^{2}}{y_{i j} y_{i k}}+\widetilde{M}_{-1-11}^{3} \frac{\mu_{q} y_{j k}}{y_{i j} y_{i k}}+\widetilde{M}_{-1-11}^{4} \frac{\mu_{q}^{2} y_{j k}}{y_{i j} y_{i k}}\right) .
\end{aligned}
$$

In a fixed order context, the corresponding massive $d_{3}^{0}$ was derived from the matrix element for the decay of a neutralino into a massive gluino and two gluons, $\tilde{\chi} \rightarrow \tilde{g} g g$ [13], using an effective Lagrangian [10].

\footnotetext{
${ }^{4}$ There is an additional ambiguity which originates from the collinear gluon singularity. In VINCIA, we use identified particles and therefore we have to distribute the collinear gluon splitting singularity $p_{j} \| p_{k}$ onto two dipole-antennae, one in which $j$ is a hard radiator and only $k$ can become soft and one in which $k$ is a hard radiator and only $j$ can become soft. When doing so, we could introduce a $j \leftrightarrow k$-asymmetric term of the form $\chi\left(y_{i j}-y_{i k}\right) /\left[y_{j k}\left(1-\mu_{q}^{2}\right)\right]$ to the dipole-antenna function which contributes in the collinear limit $j \| k$. To first order, such an asymmetric term cancels out, but it would still influence the shower at higher orders. However, since values different from $\chi=0$ tend to lead to negative dipole-antenna functions for high quark masses, we have so far not enabled the option to vary it in the VINCIA code.
} 
$\mathbf{q g} \rightarrow \mathbf{q} \overline{\mathbf{q}}^{\prime} \mathbf{q}^{\prime}$

\begin{tabular}{lrrrr}
\hline $\bar{a}_{\bar{q}^{\prime} / q g}$ & Def & GGG & MIN & MAX \\
\hline $\mathcal{C}$ & $n_{f}$ & $n_{f}$ & $n_{f}$ & $n_{f}$ \\
\hline$C_{00}$ & 0.3 & 0.5 & - & 0.6 \\
$C_{10}$ & - & - & - & - \\
$C_{01}$ & - & -0.5 & - & - \\
\hline$\widetilde{C}_{01}$ & - & -2.0 & - & - \\
$M_{00}^{10}$ & - & -1.0 & - & - \\
$M_{00}^{20}$ & - & -0.5 & - & - \\
$\widetilde{M}_{00}^{02}$ & - & -2.0 & - & - \\
$\widetilde{M}_{00}^{10}$ & - & -4.0 & - & - \\
$\widetilde{M}_{00}^{20}$ & - & -2.0 & - & - \\
\hline
\end{tabular}

gg $\rightarrow$ ḡ̄q

\begin{tabular}{lrrrr}
\hline $\bar{a}_{\bar{q} / g g}$ & Def & GGG & MIN & MAX \\
\hline $\mathcal{C}$ & $n_{f}$ & $n_{f}$ & $n_{f}$ & $n_{f}$ \\
\hline$C_{00}$ & 0.3 & - & - & 0.6 \\
$C_{10}$ & - & - & - & - \\
$C_{01}$ & - & - & - & - \\
\hline$\widetilde{C}_{00}$ & - & -2.0 & - & - \\
$\widetilde{C}_{01}$ & - & 1.0 & - & - \\
$M_{00}^{2}$ & - & 2.0 & - & - \\
\hline
\end{tabular}

Table 2: Colour factors and finite parts for four different examples of the colour-ordered $q g \rightarrow q \bar{q}^{\prime} q^{\prime}$ and $g g \rightarrow$ $g \bar{q} q$ antenna functions. The VINCIA default antenna set (Def) is compared to the "GGG", "MIN", and "MAX" variations. Coefficients which are not listed (or which are represented by "-") are zero.

Similarly to the massive dipole-antenna $\bar{a}_{g / q \bar{q}}$, the denominator factor $s_{I K}^{2}=\left(m_{I K}^{2}-m_{q}^{2}\right)^{2}$ is proportional to the coupling-stripped two-particle matrix element for neutralino decay into a gluino and a gluon, $\tilde{\chi} \rightarrow \tilde{g} g$. It has mass dimension four in this case because the neutralino-gluino-gluon coupling has mass dimension -2 . The parametrization in equation (42) contains finite terms which are proportional to $\mu_{q}^{2} /\left(y_{i j} y_{i k}\right)$. Although these terms seem out of place in a quark-gluon dipole-antenna function, they are indeed part of the fixed-order antenna $d_{3}^{0}$ if the quark is massive. Their appearance is connected to the fact that the physical matrix element for $\tilde{\chi} \rightarrow \tilde{g} g g$ from which $d_{3}^{0}$ is extracted is symmetric under the interchange of the two gluons. We are using the quark-gluon dipole-antenna function to generate emissions in a situation which is decidedly asymmetric between the two gluons since one is identified as the hard radiator and the other is the emitted particle. For this reason, the terms proportionate to $\mu_{q}^{2} /\left(y_{i j} y_{i k}\right)$ have been deactivated by default in VINCIA and are not even considered for the purpose of uncertainty estimates.

The values for the other coefficients are summarized in the right-hand pane of table 1, with the coefficients reproducing the fixed-order $d_{3}^{0}$ given in the "GGG" column.

Obviously, there are no (leading-order) mass effects for $g g \rightarrow g g g$. We include the corresponding generic $^{5}$ form of the dipole-antenna function here for completeness,

$$
\bar{a}_{g / g g}\left(m_{I K}^{2}, s_{i j}, s_{j k}\right)=\frac{1}{m_{I K}^{2}}\left(\frac{2 y_{i k}}{y_{j k} y_{i j}}+\frac{y_{i j}\left(1-y_{i j}\right)}{y_{j k}}+\frac{y_{j k}\left(1-y_{j k}\right)}{y_{i j}}+\sum_{a, b \geq 0}^{a+b \leq 2} C_{a b} y_{i j}^{a} y_{j k}^{b}\right),
$$

where the last term represents $F_{g / g g}$, with coefficients as given in the bottom left-hand pane of table 1 . The "GGG" column tabulates the coefficients of the $f_{3}^{0}$ function derived in [11] using an effective Lagrangian for the process $H \rightarrow g g g$ normalized to $H \rightarrow g g$.

\footnotetext{
${ }^{5}$ If a term $\chi\left(y_{i j}-y_{i k}\right) /\left[y_{j k}\left(1-\mu_{q}^{2}\right)\right]$ is added to equation (41), we would also need to introduce a term $\chi\left(y_{i j}-y_{i k}\right) / y_{j k}+$ $\chi\left(y_{j k}-y_{i k}\right) / y_{i j}$ in equation (43) to ensure that $a_{g / q g}\left(p_{i}, p_{j}, p_{k}\right)+a_{g / g g}\left(p_{j}, p_{k}, p_{l}\right)$ reproduces $P_{g / g g}$ for $p_{j} \| p_{k}$.
} 
We proceed with the dipole-antennae describing the gluon splitting processes. The gluon-splitting process $Q g \rightarrow Q \bar{Q}^{\prime} Q^{\prime}$ is described by the following generic form,

$$
\bar{a}_{\bar{q}^{\prime} / q g}\left(m_{I K}^{2}, s_{i j}, s_{j k}, m_{q}, m_{q^{\prime}}\right)=\frac{1}{2} \frac{1}{s_{j k}+2 m_{q^{\prime}}^{2}}\left(\frac{s_{i k}^{2}+s_{i j}^{2}}{s_{I K}^{2}}+\frac{2 m_{q^{\prime}}^{2}}{s_{j k}+2 m_{q^{\prime}}^{2}}\right)+\frac{m_{I K}^{2}}{s_{I K}^{2}} F_{\bar{q}^{\prime} / q g}
$$

with finite terms,

$$
\begin{aligned}
F_{\bar{q}^{\prime} / q g}= & \frac{y_{j k}}{y_{j k}+2 \mu_{q^{\prime}}^{2}}\left(C_{00}+C_{10} y_{i j}+C_{01} y_{j k}+C_{20} y_{i j}^{2}+C_{02} y_{j k}^{2}+C_{11} y_{i j} y_{j k}\right) \\
& +\frac{y_{j k} \mu_{q}}{y_{j k}+2 \mu_{q^{\prime}}^{2}}\left(M_{00}^{10}+M_{10}^{10} y_{i j}+M_{01}^{10} y_{j k}+\mu_{q}\left(M_{00}^{20}+M_{10}^{20} y_{i j}+M_{01}^{20} y_{j k}\right)\right) \\
& +\frac{\mu_{q^{\prime}}^{2}}{y_{j k}+2 \mu_{q^{\prime}}^{2}}\left(\widetilde{C}_{00}+\widetilde{C}_{10} y_{i j}+\widetilde{C}_{01} y_{j k}+\widetilde{C}_{20} y_{i j}^{2}+\widetilde{C}_{02} y_{j k}^{2}+\widetilde{C}_{11} y_{i j} y_{j k}\right) \\
& +\frac{\mu_{q^{\prime}}^{2} \mu_{q}}{y_{j k}+2 \mu_{q^{\prime}}^{2}}\left(\widetilde{M}_{00}^{10}+\widetilde{M}_{10}^{10} y_{i j}+\widetilde{M}_{01}^{10} y_{j k}+\mu_{q}\left(\widetilde{M}_{00}^{20}+\widetilde{M}_{10}^{20} y_{i j}+\widetilde{M}_{01}^{20} y_{j k}\right)\right) \\
& +\frac{\mu_{q^{\prime}}^{4}}{y_{j k}+2 \mu_{q^{\prime}}^{2}}\left(\widetilde{M}_{00}^{02}+\widetilde{M}_{10}^{02} y_{i j}+\widetilde{M}_{01}^{02} y_{j k}\right)
\end{aligned}
$$

whose values are listed in the left-hand pane of table 2. In a fixed order context, the massive $e_{3}^{0}$ quarkgluon antenna can be derived from the decay of a neutralino into a gluino and quark-antiquark, i.e from the process $\tilde{\chi} \rightarrow \tilde{G} Q \bar{Q}$. The resulting partons, gluino and a quark-antiquark pair can either be massless or massive. The presence of two different masses is the main reason why the structure of the finite term $F_{\bar{q}^{\prime} / q g}$ is more complicated than for the other dipole-antenna functions. The values of the coefficients which reproduce the sub-antenna $e_{3}^{0}$ for the most general case (all partons massive) are given in the GGG column of table 2 .

Contours of constant value of this function with default parameters for the finite part are illustrated in figure 4, for three different mass combinations, shown from left to right. Dashed contours represent massive functions, short dashed contours represent massless functions. Apart from the modifications to the size of the physical phase space, the mass effects are only numerically important away from the collinear limit.

Finally, the gluon-splitting process $g g \rightarrow g \bar{Q} Q$ is described by the following generic form of its dipole-antenna function

$$
\bar{a}_{\bar{q} / g g}\left(m_{I K}^{2}, s_{i j}, s_{j k}, m_{q}\right)=\frac{1}{2} \frac{1}{s_{j k}+2 m_{q}^{2}}\left(\frac{s_{i k}^{2}+s_{i j}^{2}}{s_{I K}^{2}}+\frac{2 m_{q}^{2}}{s_{j k}+2 m_{q}^{2}}\right)+\frac{m_{I K}^{2}}{s_{I K}^{2}} F_{\bar{q}^{\prime} / q g},
$$




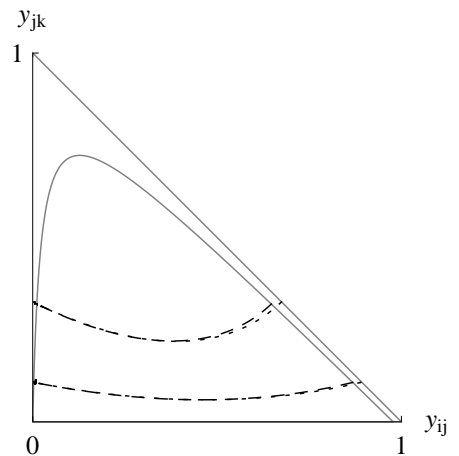

(a) $Q g \rightarrow Q \bar{q}^{\prime} q^{\prime}$

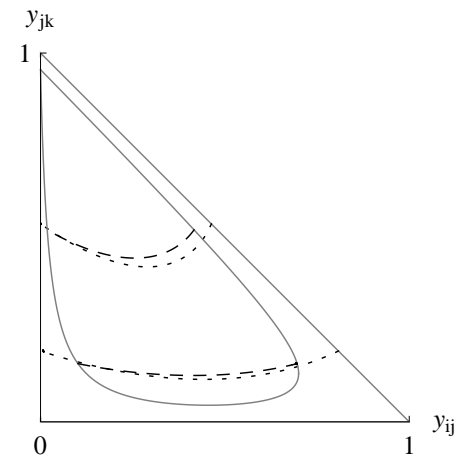

(b) $q g \rightarrow q \bar{Q}^{\prime} Q^{\prime}$

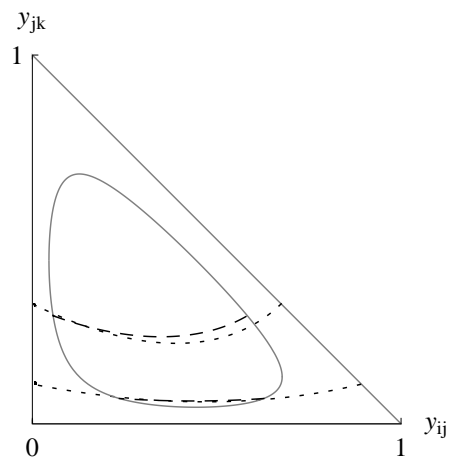

(c) $Q g \rightarrow Q \bar{Q}^{\prime} Q^{\prime}$

Figure 4: Dalitz plot showing contours of the massive (dashed) and massless (short dashed) gluon-splitting antenna function $\bar{a}_{\bar{q}^{\prime} / q g}$, for three different combinations of massive and massless partons, with $m_{Q}=0.15 m_{I K}$. Contours are shown for $\bar{a}=1,4$ in (a) and (c) and for $\bar{a}=0.5,2$ in (b). Grey solid lines mark the boundary of the phase space. Contrary to the gluon emission case, there are no qualitative changes caused by the introduction of quark masses.

with finite terms,

$$
\begin{aligned}
F_{\bar{q} / g g}= & \frac{y_{j k}}{y_{j k}+2 \mu_{q}^{2}}\left(C_{00}+C_{10} y_{i j}+C_{01} y_{j k}+C_{20} y_{i j}^{2}+C_{02} y_{j k}^{2}+C_{11} y_{i j} y_{j k}\right) \\
& +\frac{\mu_{q}^{2}}{y_{j k}+2 \mu_{q}^{2}}\left(\widetilde{C}_{00}+\widetilde{C}_{10} y_{i j}+\widetilde{C}_{01} y_{j k}+\widetilde{C}_{20} y_{i j}^{2}+\widetilde{C}_{02} y_{j k}^{2}+\widetilde{C}_{11} y_{i j} y_{j k}\right) \\
& +\frac{\mu_{q}^{4}}{y_{j k}+2 \mu_{q}^{2}}\left(M_{00}^{2}+M_{10}^{2} y_{i j}+M_{01}^{2} y_{j k}\right) .
\end{aligned}
$$

In this case, the corresponding fixed-order antenna $g_{3}^{0}$ can be derived from the decay of a Higgs into a gluon and a massive quark-antiquark pair i.e from $H \rightarrow Q \bar{Q} g$. It can be obtained from the dipoleantenna analogue given in equation (46) by setting the finite parts $\mathrm{F}$ according to the right-hand pane of table 2.

Note that the finite parts of the the two dipole-antennae related to gluon splitting $\bar{a}_{\bar{q}^{\prime} / q g}$ and $\bar{a}_{\bar{q} / g g}$ have been parametrized in the same way. In the $\bar{a}_{\bar{q} / g g}$ case, some simplifications occur though due to the presence of a massless parton in the final state.

Finally, let us mention that VINCIA is not necessarily restricted to describe processes in the Standard Model with massive fermions in the final state. It could equally be used to describe processes with massive final state particles with different spin-statistics properties. Since many models of physics beyond the Standard Model contain new heavy coloured particles which can be scalars, VINCIA needs a default dipole-antenna function for those. Since the soft Eikonal factor of equation (30) is spinindependent, it can be used as a default dipole-antenna in VINCIA for those cases:

$$
\bar{a}_{\text {Eikonal }}\left(m_{I K}^{2}, s_{i j}, s_{j k}, m_{I}^{2}, m_{K}^{2}\right)=\frac{2 s_{i k}}{s_{i j} s_{j k}}-\frac{2 m_{I}^{2}}{s_{i j}^{2}}-\frac{2 m_{K}^{2}}{s_{j k}^{2}},
$$


where $m_{I}=m_{i}$ and $m_{K}=m_{k}$ are the masses of the radiating partons and $s_{i k}$ can be obtained from the other arguments using equation (2). The colour factor used is equal to $\hat{C}_{F}=2 C_{F}$ if both parents are in the fundamental representation, $C_{A}$ if both are adjoints, and options for anything in between for mixed-parent antennae, with the default being $\frac{1}{2}\left(\hat{C}_{F}+C_{A}\right)$.

\section{The VINCIA Formalism with Massive Particles}

In this section we present the main ingredients of our dipole-antenna shower formalism implemented in the present version of VINCIA. This formalism was first derived in $[5,6]$ to describe QCD radiation off massless partons. We here generalize it to take quark mass effects into account. For completeness, some aspects which are carried over from the massless case without modification are also summarized. ${ }^{6}$

VINCIA is based on the dipole-antenna picture of QCD radiation [27]: its fundamental evolution step is a Lorentz-invariant $2 \rightarrow 3$ branching process $I K \rightarrow i j k$ by which two on-shell parent partons $(I$ and $K)$ are replaced by three on-shell daughter partons $(i, j, k)$, conserving four-momentum exactly. Dipole-antenna functions and phase-space mappings were discussed in sections 2.3 and 2.5.

To construct an explicit shower algorithm, one must furthermore introduce an evolution variable (a.k.a. shower ordering variable or resolution scale), $Q_{E}$, suitably generalized to massive particles as will be discussed in section 3.1. Together with this variable one also needs to define a concrete iterative strategy for generating and accepting "trial branchings" according to the Sudakov form factors, as described in sections $3.2-3.4$.

Matching to fixed-order matrix elements is performed using a unitary matching scheme, which results in unweighted events matched to full-colour tree-level matrix elements squared, as explained in [6]. We give a brief summary of the main points of this method in section 3.5. VINCIA also calculates uncertainty estimates for its predictions at a moderate speed penalty, those are summarized in section 3.6.

\subsection{Ordering}

In the dipole-antenna formalism, subsequent emissions are naturally ordered by the nesting of the onshell $2 \rightarrow 3$ phase spaces: as more and more emissions (or $g \rightarrow q \bar{q}$ splittings) are added, each dipoleantenna will, on average, carry a progressively smaller fraction of the total original centre-of mass energy squared $s$. We refer to this as "no ordering", but it could equally well be called "phase-space-ordering", since the only constraint implied is that energy-momentum inside the nested $2 \rightarrow 3$ phase spaces is conserved.

As demonstrated in [6,37], however, and further elaborated on for the massive case in our section 4, a dipole-antenna shower without any additional constraints would produce far too much radiation outside the double-logarithmic limit, i.e in the so-called "hard region". This is essentially due to the fact that an "unordered" shower approximation can be viewed as a sum of independent dipoles, while full QCD, beyond the $2 \rightarrow 3$ level, has a more complicated multipole structure, with in particular destructive interference produced by colour-coherence effects. A reasonable agreement with all-orders QCD can be restored by enforcing a strict ordering of the emissions in terms of some "evolution scale", $Q_{E}$. This variable represents a measure of (inverse) formation time or characteristic wavelength.

\footnotetext{
${ }^{6}$ We encourage readers unfamiliar with shower formulations to consult $[3,36]$ for recent pedagogical reviews.
} 
The VINCIA formalism can accommodate a whole class of evolution variables which differ in how they prioritize soft emissions relative to collinear ones. We refrain from characterizing all possible evolution variables here, referring instead to the original publication [6] for details.

The two most important evolution variables implemented in VINCIA are the following:

$$
Q_{E}^{2}=4 p_{\perp}^{2} \stackrel{\text { def }}{=} 4 \frac{s_{i j} s_{j k}}{m_{I K}^{2}}=4 \frac{\left(2 p_{i} \cdot p_{j}\right)\left(2 p_{j} \cdot p_{k}\right)}{m_{I K}^{2}} \stackrel{m_{j}=0}{=} 4 \frac{\left(m_{i j}^{2}-m_{i}^{2}\right)\left(m_{j k}^{2}-m_{k}^{2}\right)}{m_{I K}^{2}}
$$

(Type 2) Dipole Virtuality

$$
Q_{E}^{2}=m_{D}^{2} \stackrel{\text { def }}{=} 2 \min \left(s_{i j}, s_{j k}\right) \stackrel{m_{j}=0}{=} 2 \min \left(m_{i j}^{2}-m_{i}^{2}, m_{j k}^{2}-m_{k}^{2}\right),
$$

where we again emphasize that the notation $s_{i j}$ is used for the dot product $s_{i j}=2 p_{i} \cdot p_{j}$, which differs from the invariant mass squared $m_{i j}^{2}=\left(p_{i}+p_{j}\right)^{2}$ when non-zero rest masses are involved. Note that the rightmost expressions in the above equations are appropriate only to gluon emission, for which $m_{j}=0$.

While the imposition of such ordering conditions can extend the agreement with higher-order QCD to a much larger region of phase space outside the double-logarithmic limit (see, e.g., [6,37]), it does have a side effect, which is formally beyond LL: in general, there will be small corners of the $n$-particle phase space which are not accessible through any sequence of strongly ordered branchings $[37,38]$. Those are called "dead zones", which the shower does not populate at all. These zones correspond to regions of phase space that are classified by the ordering condition as having no LL contributions. It is therefore consistent to set these corresponding contributions to zero at the LL level.

As shown in [6], it is possible to avoid dead zones without re-introducing the large overestimates present in the "no-ordering" scenario. This can be done by allowing unordered branchings to occur with a suppressed probability which does not affect the LL accuracy of the shower. This is technically achieved by starting from an unordered shower and, instead of applying the strong-ordering condition as a step function, apply a smooth damping factor instead. We label this as an "improved" ordering condition, $P_{\text {imp }}$,

$$
\Theta_{\text {strong-ordering }} \rightarrow P_{\text {imp }}\left(\widehat{Q}_{E}^{2}, Q_{E}^{2}\right)=\frac{\widehat{Q}_{E}^{2}}{\widehat{Q}_{E}^{2}+Q_{E}^{2}},
$$

where $Q_{E}$ is the evolution scale of the current $n \rightarrow n+1$ branching. $\widehat{Q}_{E}$ is a measure of the scale of the $n$-particle configuration, defined as the minimal value of the evolution scale evaluated over all partons in the $n$-parton parent configuration. For branchings which are at a much lower scale than the last one, i.e. the strongly ordered limit $Q_{E}^{2} \ll \widehat{Q}_{E}^{2}$, this factor is unity, whereas branchings with the opposite hierarchy $Q_{E}^{2} \gg \widehat{Q}_{E}^{2}$ are strongly suppressed. At the point $Q_{E}^{2}=\widehat{Q}_{E}^{2}$, the suppression factor in equation (51) is equal to $1 / 2$. We refer to this as the "smooth ordering" condition.

Note that, since the $P_{\text {imp }}$ factor is everywhere smaller than unity, it can be applied as a probabilistic veto, which we make use of in the technical implementation.

\subsection{The Evolution Algorithm}

Formally, we can define a unitary evolution operator $\mathcal{S}\left(\{p\}_{n}, Q_{\text {start }}, Q_{\text {stop }}\right)$ which generates the perturbative radiation off an $n$-parton state $\{p\}_{n}$ between the two resolution scales $Q_{\text {start }}$ and $Q_{\text {stop }}$ in terms of 
an iterative Markov chain ${ }^{7}$

$$
\begin{aligned}
& \mathcal{S}\left(\{p\}_{n}, Q_{\text {start }}, Q_{\text {stop }}\right)=\Delta\left(\{p\}_{n}, Q_{\text {start }}, Q_{\text {stop }}\right) \\
& \quad+\sum_{I K \rightarrow i j k} \int_{Q_{\text {stop }}}^{Q_{\text {start }}} \frac{\mathrm{d} \Phi_{3}^{i j k}}{\mathrm{~d} \Phi_{2}^{I K}} a_{j / I K} \Delta\left(\{p\}_{n}, Q_{\text {start }}, Q^{i j k}\right) \mathcal{S}\left(\{p\}_{n+1}^{I K \rightarrow i j k}, Q_{\text {restart }}^{i j k}, Q_{\text {stop }}\right),
\end{aligned}
$$

where the first line represents the fraction of states that remain unchanged by the evolution (i.e., the exclusive $n$-parton fraction at the resolution scale $Q_{\text {stop }}$ ), while the second line includes all states that do evolve (i.e., the inclusive $(n+1)$-parton fraction). To maintain unitarity, the second line must be minus the derivative of the first. Analogously to other time-dependent decay problems, the solution to this differential equation is that the Sudakov factor, $\Delta$, must be the exponential of minus the integrated tree-level branching probability,

$$
\begin{gathered}
\Delta\left(\{p\}_{n}, Q_{\text {start }}, Q_{\text {emit }}\right)=\prod_{I K \rightarrow i j k} \exp \left(-\mathcal{A}_{j / I K}\left(Q_{\text {start }}, Q_{\text {emit }}\right)\right), \\
\mathcal{A}_{j / I K}\left(Q_{\text {start }}, Q_{\text {emit }}\right)=\int_{Q_{\text {emit }}}^{Q_{\text {start }}} \frac{\mathrm{d} \Phi_{3}^{i j k}}{\mathrm{~d} \Phi_{2}^{I K}} a_{j / I K}\left(p_{i}, p_{j}, p_{k}\right),
\end{gathered}
$$

with phase-space measures and dipole-antenna functions as defined in section 2.

The sum in equation (52) and the product in (53) run over all possible $2 \rightarrow 3$ branchings $I K \rightarrow i j k$. The integrals in equations (52) and (54) are performed over the range $Q_{\text {start }}>Q^{i j k}>Q_{\text {stop }}$, with $Q^{i j k}=Q_{E}\left(p_{i}, p_{j}, p_{k}\right)$ the evolution variable.

The starting scale, $Q_{\text {start }}$, represents the "factorization scale" of the $n$-parton configuration and may be given either by the invariant mass of the evolving dipole-antenna, by the restart scale defined by a previous branching, or by some externally imposed scale, depending on the type of ordering criterion imposed on the shower evolution.

In a standard shower application, $Q_{\text {stop }}$ represents the infrared shower cutoff, or hadronization scale, with a value of $\sim 1 \mathrm{GeV}$. For simplicity, we have assumed here that the definition of $Q_{\text {stop }}$ in terms of the post-branching momenta $p_{i}, p_{j}$ and $p_{k}$ is the same as that of $Q_{\mathrm{emit}}$. In practice, this is not necessarily the case, see the section on hadronization in [6].

The presence of $\mathcal{S}\left(\{p\}_{n+1}^{I K \rightarrow i j k}, Q_{\text {restart }}^{i j k}, Q_{\text {stop }}\right)$ in equation (52) generates the continued (iterated) evolution of the $(n+1)$-parton state after branching, with $Q_{\text {restart }}^{i j k}$ normally taken to be equal to $Q^{i j k}$, for a traditional so-called strongly-ordered shower.

Now we turn to the algorithmic steps themselves. Since the $2 \rightarrow 3$ branching phase space is threedimensional (two independent Lorentz invariants, e.g. $s_{i j}$ and $s_{j k}$, and the azimuthal angle $\phi$ which determines the global orientation), three independent random numbers must be picked for each step of the algorithm. The first of these is the $Q_{\text {emit }}$ scale, distributed according $1-\Delta\left(Q_{\text {start }}, Q_{\text {emit }}\right)$ with the "no-emission" probability $\Delta$ given in equation (53). Next, the second independent Lorentz invariant must be generated. It is generated according to the integrand in equation (54). From the two Lorentz invariants and the azimuthal angle $\phi$ (which is chosen according to a flat probability density because the

\footnotetext{
${ }^{7}$ Strictly speaking, strongly ordered showers depend on the scale of the last branching, and are therefore not completely Markovian. In the context of VINCIA, the difference is only really relevant in the context of matching to matrix elements and can therefore be ignored for the discussion of the pure shower algorithm.
} 
integrand does not depend on it), the momenta $p_{i}, p_{j}$, and $p_{k}$ can then be constructed from the parent momenta $p_{I}$ and $p_{K}$, based on the chosen kinematics map and the relations given in section 2.3.

The algebra and computational overhead involved in generating the three branching invariants can be simplified considerably by judicious use of the veto algorithm (see $[3,39,40]$ for pedagogical reviews). First, so-called "trial branchings" are generated, using a simplified form of the integrand in equation (54). A percentage of these trials are then rejected, using

$$
P_{\text {accept }}=\frac{a_{j / I K}\left(m_{I K}^{2}, s_{i j}, s_{j k},\{m\}\right)}{a_{\text {trial }}\left(m_{I K}^{2}, s_{i j}, s_{j k}\right)},
$$

to determine whether a given trial branching should be accepted or not. Symbolically $\{m\}$ stands for all the masses of the parent and daughter partons, $a_{j / I K}$ is the desired integrand in equation (54), and $a_{\text {trial }}$ is the simplified trial function. The veto algorithm ensures that the final answer has no dependence (apart from the speed with which it is obtained) on the form of the trial function used, requiring only that this function is an overestimate of the correct integrand over all of phase space, so that the accept probability equation (55) does not exceed unity.

\subsection{Trial Gluon Emissions}

For gluon emission, the trial function used in VINCIA is based on the double-pole singular behaviour of the soft Eikonal factor, equation (30), which it coincides with in the soft limit and overestimates everywhere else. Using the notation conventions adopted in section 2.1, it can be adapted straightforwardly from the massless case, and is given by

$$
a_{\text {trial-emit }}=\frac{\hat{\alpha}_{S}}{4 \pi} C_{A} \frac{2 m_{I K}^{2}}{s_{i j} s_{j k}},
$$

where the overestimates $\hat{\alpha}_{S} \geq \alpha_{S}$ and $C_{\text {trial-emit }}=C_{A} \geq \mathcal{C}_{j / I K}$ can be used to guarantee sufficient "headroom" for arbitrary coupling constants and colour factors, respectively. Since the mass corrections to the soft Eikonal factor and to the quasi-collinear splitting function are negative (as given in equations (30) and (35) and illustrated, e.g., in figure 2), this function is also guaranteed to be an overestimate in the massive case.

In figure 5, we attempt to give a more concrete impression of the suitability of this trial function, for a $b \bar{b} \rightarrow b g \bar{b}$ branching in a dipole-antenna of mass $\sqrt{s}=91 \mathrm{GeV}$ (left pane) and $=45.5 \mathrm{GeV}$ (right pane), with $m_{b}=4.8 \mathrm{GeV}$ in both cases and a gluon energy of $E_{g}=10 \mathrm{GeV}$. These values were selected so as to yield plots that can be compared directly to those in [4]. The $x$ axes show the gluon emission angle in degrees, going from the collinear limit (zero angle) at origin to a 90-degree emission angle on the right-hand edge of the plots. The value of the gluon-emission trial function defined in equation (56) is shown as a thick solid black line. It is everywhere larger than all the other curves and hence represents an overestimate, as desired. The Eikonal factor defined in equation (30) is shown as a thin solid line (red). The default VINCIA $q \bar{q}$ antenna function defined in equation (40) is shown as a thick lighter (yellow) curve; it is slightly larger than the Eikonal factor, but is still everywhere smaller than the trial function. For completeness, antenna functions derived from two different LO matrix elements are also shown (for $H^{0}$ and $Z^{0}$ decay, shown with dots and dashes, respectively). Note that the matrix-element curves are closer to the default VINCIA antenna function than to the Eikonal factor, in particular in the 

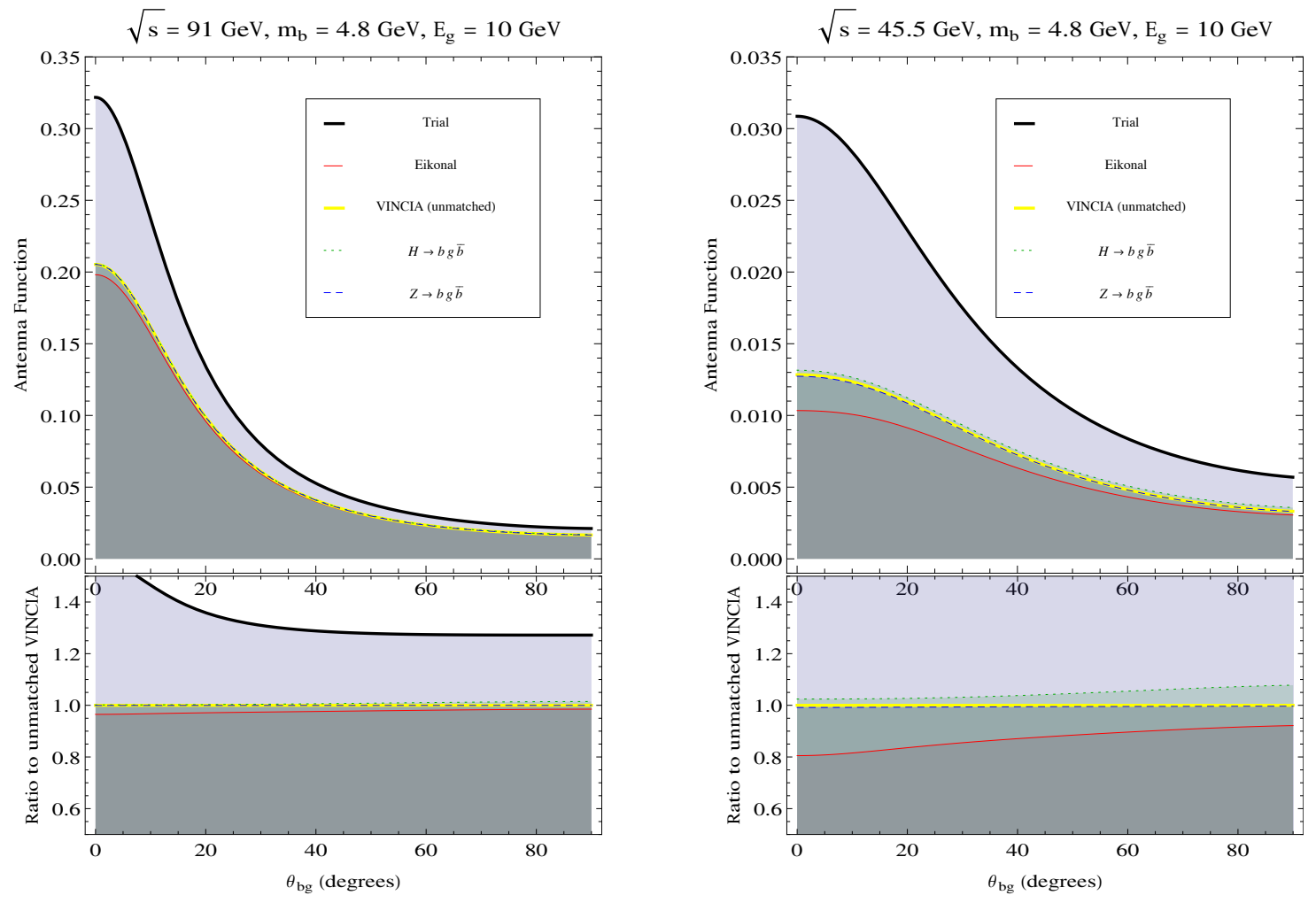

Figure 5: Various levels of approximation for $Q \bar{Q} \rightarrow Q g \bar{Q}$, compared to the VINCIA trial and default (unmatched) shower functions. Left: the size of the dipole-antenna functions as a function of emission angle for a gluon energy of $10 \mathrm{GeV}$, for $\sqrt{s}=91 \mathrm{GeV}$ and $m_{Q}=4.8 \mathrm{GeV}$. Right: the same for $\sqrt{s}=45.5 \mathrm{GeV}$. Lower panes show results normalized to the default VINCIA dipole-antenna function.

zero-degree region. This is due to the fact that the default VINCIA antenna function not only reproduces the soft limit, but also the quasi-collinear limit of the full matrix elements.

Note also that the trial function is closest to the physical antenna functions in the left-hand pane, where the values of the antenna functions are ten times larger than in the right-hand one (notice the factor 10 difference in the $y$ axis scales). The overall efficiency of the trial algorithm, which is dominated by the regions in which the trial function is large, is hence quite reasonable.

The corresponding evolution integral for trial gluon emission, equation (54), was defined for massless partons in [6], in terms of the variables $Q_{E}$ and $\zeta$,

$$
\mathcal{A}_{\text {trial-emit }}\left(Q_{\text {start }}, Q_{\text {emit }}\right)=C_{A} \int_{Q_{\text {emit }}^{2}}^{Q_{\text {start }}^{2}} \mathrm{~d} Q_{E}^{2} \mathrm{~d} \zeta|J| \frac{\hat{\alpha}_{S}\left(Q_{E}^{2}\right)}{4 \pi} \frac{2}{s_{i j}\left(Q_{E}^{2}, \zeta\right) s_{j k}\left(Q_{E}^{2}, \zeta\right)} .
$$

We have suppressed a trivial integration over $\phi$ and changed variables from $s_{i j}$ and $s_{j k}$ to $Q_{E}^{2}$ and an arbitrary (linearly independent) phase-space variable $\zeta$, with $|J|$ the Jacobian of the transformation. For the two $Q_{E}$ definitions discussed in section 3.1, a convenient definition of $\zeta$ is

$$
\zeta=\frac{s_{i j}}{s_{i j}+s_{j k}} .
$$


For both of the evolution variables considered above, the inverse relations can be written

$$
s_{i j}=\zeta \tilde{Q}^{2}, \quad s_{j k}=(1-\zeta) \tilde{Q}^{2},
$$

with the definition of $\tilde{Q}$ depending on the choice of evolution variable. For $Q_{E}=2 p_{\perp}$,

$$
\tilde{Q}^{2}=\frac{Q_{E} m_{I K}}{2 \sqrt{\zeta(1-\zeta)}}
$$

while for $Q_{E}=m_{D}$,

$$
\tilde{Q}^{2}=\frac{Q_{E}^{2}}{\min (\zeta, 1-\zeta)} .
$$

Using these relations, a set of $\left(Q_{E}, \zeta\right)$ values can be translated unambiguously back to the original phase-space invariants $\left(s_{i j}, s_{j k}\right)$. We therefore emphasize that, although the $\zeta$ variable plays a role analogous to the $z$ fraction of traditional parton showers, it here serves merely to (re)parametrize phase space; there is no explicit dependence on this choice [6].

Since the massive phase space is contained within the massless one, the massless phase space generator developed in [6] can be recycled for massive momenta. In this case, the points which do not correspond to physical massive momenta are rejected. We do have to take into account, however, that the two-particle phase space $\mathrm{d} \Phi_{2}\left(\mathrm{p}_{\mathrm{I}}, \mathrm{p}_{\mathrm{K}}\right)$, which normalizes the the dipole-antenna branching phase space, depends on the masses of the parent partons $I$ and $K$ and is not anymore just given by the invariant mass of the parent partons as in the massless case. Instead, we have

$$
\sqrt{\lambda\left(1, \mu_{I}^{2}, \mu_{K}^{2}\right)} \frac{\mathrm{d} \Phi_{3}\left(p_{i}, p_{j}, p_{k}\right)}{\mathrm{d} \Phi_{2}\left(p_{I}, p_{K}\right)}=\left.\frac{\mathrm{d} \Phi_{3}\left(p_{i}, p_{j}, p_{k}\right)}{\mathrm{d} \Phi_{2}\left(p_{I}, p_{K}\right)}\right|_{m_{I}=m_{K}=0}
$$

where the Källén function $\lambda$ is defined in equation (8) and the reduced masses are defined as $\mu=$ $m / m_{I K}$, with $m_{I K}^{2}=\left(p_{I}+p_{K}\right)^{2}$. In the massive case, the relevant evolution integral can therefore be written as the massless evolution integral modified by a massive phase space factor,

$$
\mathcal{A}_{\text {trial-emit }} \rightarrow \frac{\left.\mathcal{A}_{\text {trial-emit }}\left(m_{I K}^{2}, Q_{\text {start }}, Q_{\text {emit }}\right)\right|_{m_{I}=m_{K}=0}}{\sqrt{\lambda\left(1, \mu_{I}^{2}, \mu_{K}^{2}\right)}} .
$$

We take this into account by applying the Källén factor as a multiplicative pre-factor on the trial emission probabilities. With this replacement, the treatment of trial gluon emission derived in [6] in the massless case, can be carried over to the massive one without further modifications.

\subsection{Trial Gluon Splittings}

For gluon-splitting antennae, i.e. those containing a $g \rightarrow q \bar{q}$ branching, we again make use of the leading singularity structure of the underlying process. This improves on the treatment in [6], in which equation (56) was used for both gluon emission and gluon splitting. Irrespective of whether a gluon splits into a massive or into a massless quark-antiquark pair, the process is characterised by an $s$-channel gluon propagator with a singularity structure given by the factor $1 / m_{q \bar{q}}^{2}$. The effect of non-zero quark masses is only to restrict the emission phase space in such a way that the $m_{q \bar{q}} \rightarrow 0$ singularity present in the 
massless case cannot be reached with on-shell quarks. This suggests that the "optimal" trial function to use for gluon splitting processes (massless and massive) is:

$$
a_{\text {trial-split }}=\frac{\hat{\alpha}_{S}}{4 \pi} n_{f} \widehat{T}_{R} \frac{1}{m_{q \bar{q}}^{2}},
$$

where $n_{f}$ denotes the number of active flavours and we recall that $\widehat{T}_{R}=1$ in our conventions.

While different choices for the evolution variable for gluon emissions are implemented in VINCIA, the evolution variable for gluon splitting has been fixed to gluon virtuality, $m_{q \bar{q}}^{2}$, as also advocated in [41]. This is based on the fact that, while gluon emission involves a sum over terms that have different soft and collinear limits, with different evolution variables assigning different "times" to each region, here there is only one singular structure, in $m_{q \bar{q}}^{2}$, and hence we consider this choice to be relatively unambiguous. Further motivation is provided by a comparison between mass- and $p_{\perp}$-ordering given in section 4.2.

What remains ambiguous is then the details of how to "interleave" gluon emissions and gluon splittings in the parton shower evolution. The VINCIA algorithm sketched in this section is based on generating a branching scale for every possible $2 \rightarrow 3$ branching and choosing the highest of those scales to determine which branching occurs next. We therefore need a way to determine whether a possible gluon splitting is at a higher scale than a possible gluon emission. Once we have defined how we compare a gluon splitting scale with a gluon emission scale, the interleaving of gluon emission and gluon splitting works exactly the same way as the interleaving of gluon emissions from different dipole-antennae. In order to compare the scales, we use the fact that all gluon emission evolution variables as well as the evolution variable for gluon splitting, $m_{q \bar{q}}$, range from 0 for strictly soft/collinear branchings to $m_{I K}$ for the hardest branching process kinematically allowed. This suggests that we can compare $m_{q \bar{q}}$ directly with the gluon emission variable, for example $2 p_{\perp}$. There is an ambiguity however in how we define this comparison. One could for example equally well compare $m_{I K}\left(m_{q \bar{q}} / m_{I K}\right)^{a}(a>0)$ with the gluon emission variable. In VINCIA, we have so far chosen to maintain the nominal evolution variable, with $a=1$.

Let us here consider the case where parton $K$ is the splitting gluon (as in $q g \rightarrow q \bar{q}^{\prime} q^{\prime}$ or $g g \rightarrow g \bar{q} q$ ), parton $I$ can be either massless or massive. The direct equivalent of equation (57), the integrated trial antenna function for gluon splittings, is then

$$
\mathcal{A}_{\text {trial-split }}\left(m_{I K}, Q_{\text {start }}, Q_{\text {emit }}\right)=n_{f} \widehat{T}_{R} \frac{1}{m_{I K}^{2}-m_{I}^{2}} \int_{Q_{\text {emit }}^{2}}^{Q_{\text {start }}^{2}} \mathrm{~d} s_{g \bar{q}} \mathrm{~d} s_{q \bar{q}} \frac{\hat{\alpha}_{S}}{4 \pi} \frac{1}{m_{q \bar{q}}^{2}}
$$

where $m_{I K}^{2}-m_{I}^{2}$ originates from the two-particle phase space volume (we have inserted $m_{K}=m_{g}=0$ ) and $1 / m_{q \bar{q}}^{2}$ corresponds to the trial function for the gluon splitting branching. Since we fix $Q_{E}^{2}=m_{q \bar{q}}^{2}$, the most convenient definition for $\zeta=\zeta_{\text {split }}$ is simply the other phase space invariant in the 3-particle phase space $\mathrm{d} \Phi_{3}$, normalized by the mass of the mother dipole-antenna,

$$
\zeta_{\text {split }}=\frac{m_{g \bar{q}}^{2}}{m_{I K}^{2}}
$$

with the massless phase-space boundaries

$$
\zeta_{\min }\left(y_{E}\right)=0 \quad, \quad \zeta_{\max }\left(y_{E}\right)=1-\sqrt{y_{E}} .
$$


with, $y_{E}=Q_{E}^{2} / m_{I K}^{2}=m_{q \bar{q}}^{2} / m_{I K}^{2}$ for our specific choice of evolution variable. We recall that the definition of $\zeta$ has no physical significance in our formalism (neither for gluon emission nor for the gluon splitting process).

As in the procedure for trial gluon emissions described in detail in [6] and adapted for the massive case in section 3.3, we replace the upper limit on $\zeta$ by an overestimate during trial generation,

$$
\hat{\zeta}_{\max }=\left.\zeta_{\max }\left(Q_{E \min }^{2}\right)\right|_{m_{q}=0},
$$

such that the $\zeta$ integral in a given "evolution window" (with lower boundary $Q_{E \min }$ ) becomes simply a constant

$$
I_{\zeta}\left(Q_{E}^{2}\right)=\int_{0}^{\hat{\zeta}_{\max }} \mathrm{d} \zeta=\hat{\zeta}_{\max }=1-\sqrt{\frac{Q_{E \min }^{2}}{m_{I K}^{2}}}
$$

The evolution integral, equation (65), then acquires the form

$$
\mathcal{A}_{\text {trial-split }}\left(m_{I K}, Q_{\text {start }}, Q_{\text {emit }}\right)=n_{f} \widehat{T}_{R} \frac{1}{1-\mu_{I}^{2}} \hat{\zeta}_{\text {max }} \int_{Q_{\text {start }}^{2}}^{Q_{\text {emit }}^{2}} \frac{\mathrm{d} Q_{E}^{2}}{Q_{E}^{2}} \frac{\hat{\alpha}_{S}}{4 \pi}
$$

where $\mu_{I}=\frac{m_{I}}{m_{I K}}$. If $\hat{\alpha}_{s}$ does not depend on $Q_{E}$, then the integrated trial function in equation (70) simplifies to

$$
\mathcal{A}_{\text {trial-split }}\left(m_{I K}, Q_{\text {start }}, Q_{\text {emit }}\right)=n_{f} \widehat{T}_{R} \frac{1}{1-\mu_{I}^{2}} \frac{\hat{\alpha}_{S}}{4 \pi} \hat{\zeta}_{\text {max }} \ln \left(\frac{Q_{\text {emit }}^{2}}{Q_{\text {start }}^{2}}\right)
$$

while if we consider a first-order running $\alpha_{s}$ as a function of $\mu_{R}^{2}=k_{\mu} Q_{E}^{2}=k_{\mu} m_{q \bar{q}}^{2}$, then the integral becomes

$$
\mathcal{A}_{\text {trial-split }}\left(m_{I K}, Q_{\text {start }}, Q_{\text {emit }}\right)=\frac{n_{f} \widehat{T}_{R}}{4 \pi} \frac{1}{1-\mu_{I}^{2}} \hat{\zeta}_{\max } \frac{1}{b_{0}} \ln \left(\frac{\ln \left(k_{\mu}^{2} Q_{\text {start }}^{2} / \Lambda^{2}\right)}{\ln \left(k_{\mu}^{2} Q_{\mathrm{emit}}^{2} / \Lambda^{2}\right)}\right)
$$

where the $[\ln (\ln ())]$ structure seen in equation (72) reflects the single logarithms generated by the antenna-function singularities folded with the logarithm coming from the running of $\alpha_{s}$.

In our treatment of flavour thresholds, a heavy flavour is treated as active, i.e. it contributes to the running of $\alpha_{S}$ and is allowed to be created in gluon splittings as long as we have $m_{q \bar{q}} \geq m_{Q}$ in gluon splittings and as long as $Q_{E} \geq m_{Q}$ - which is $2 p_{\perp} \geq m_{Q}$ in the default settings - in gluon emissions. The threshold $m_{q \bar{q}}=m_{Q}$ chosen here instead of the kinematical threshold $m_{q \bar{q}}=2 m_{Q}$ is a consequence of our interleaving of gluon emissions and gluon splittings discussed above combined with the gluon emission threshold choice made in [6]. Of course the kinematical conditions for the secondary production of the heavy flavour are always enforced. Therefore the only consequence of the fact that our flavour threshold is below the kinematical threshold is a slight loss of efficiency of the algorithm for $m_{Q} \leq m_{q \bar{q}} \leq 2 m_{Q}$ due to vetoed gluon splittings into the kinematically disallowed heavy flavour region. The alternative of adjusting the gluon emission flavour thresholds such that the gluon splitting thresholds are at $m_{q \bar{q}}=2 m_{Q}$ - which would be equally valid at leading logarithmic accuracy - is not implemented at present in VINCIA.

With this definition of $\mathcal{A}$ given in equation (70), the generation of trial branchings can be carried over from the formalism presented in [6]. 


\subsection{Matrix-Element Corrections (Matching)}

The procedure for matching VINCIA to leading-order matrix elements [6] is not affected by the presence of mass terms and can be adapted to the massive case by just upgrading the massless matrix elements and antenna functions to massive ones. In accordance with the antenna-factorization formalism, all particles are treated as being on shell, both in the antenna functions and in the matrix elements.

Briefly summarized, the strategy is as follows. Similarly to the PYTHIA [42] and GENEVA [43] approaches, the VINCIA matching formalism relies on the antenna shower itself to provide an allorders phase-space generator that captures the leading behaviour of full QCD by construction. At each trial branching in the shower, the accept/reject probability can then be augmented by a multiplicative factor that goes to unity in the collinear and soft limit, but which modifies the branching probability outside those limits. The modification factor is constructed precisely such that the full-colour leadingorder matrix element squared is obtained after summing over shower histories. The approach relies heavily on unitarity and is qualitatively different from other multi-leg approaches in the literature, such as the MLM (see [21] for a description) and CKKW [16] ones. An important technical difference is that VINCIA only requires a Born-level phase-space generator, with all higher multiplicities being generated by the shower. There is therefore no need for separate phase-space generators for the higher-multiplicity matrix elements, which can result in significant speed gains, both in terms of initialization time (virtually zero in VINCIA), and in terms of running speed. The reader is referred to [6] for further details.

\subsection{Uncertainty Estimates}

Another crucial point concerns how to estimate reliably the accuracy of the resulting calculation. Arguing that variations only of the renormalization scale is insufficient at best (and misleading at worst), a more comprehensive approach for all-orders (matched-shower) perturbative calculations was proposed in [6] and implemented in VINCIA. As with the prescription for matrix-element matching, this approach can again be adapted to the massive case straightforwardly.

Briefly summarized, VINCIA is able to compute a number of weights corresponding to alternative shower settings along with each event. The central weight, corresponding to the current user settings, is unity, while each of the alternative weights represents the relative probability that the event would have been produced when running with the corresponding alternative setting. The uncertainties are evaluated in a way that explicitly preserves unitarity, and hence the weights for a particular alternative setting average to 1 over a large number of events.

The uncertainties accounted for in the present version of VINCIA do not differ from those presented for the original formulation [6]. Those include, for each branching: variation of the renormalization scale by a factor of 2 in either direction, variation of the non-singular terms in the antenna functions from a "MIN" setting to a "MAX" setting (see section 2.5), variation of the shower evolution variable between $p_{\perp}$-like and mass-like choices, and variations proportional to $1 / N_{C}^{2}$. In the context of matrixelement corrections (see above), variations of the numerical value of any "matching scale" applied can also be included. See [5,6] for further details. 


\section{Comparison to Fixed-Order Matrix Elements}

By construction, the massive dipole-antenna shower formalism presented in the preceding sections reproduces the (quasi-)collinear and soft limits of the amplitude squared for a single shower branching. In this section we present some examinations of its behaviour across multiple (combinations of) gluon emissions and/or gluon splittings. Specifically, we compare tree-level expansions of the shower to fixedorder matrix elements for $Z \rightarrow 4,5$ and 6 partons, treating each (leading-)colour structure separately ${ }^{8}$.

We consider three possible evolution orderings: no ordering, strong ordering in transverse momentum and smooth ordering in transverse momentum, as defined in section 3.1. A comparison with other orderings can be found in [6] for massless partons. For gluon splitting, we also consider the difference between ordering in transverse momentum and ordering in gluon virtuality. The dipole-antenna functions are the default ones given in section 2.5, with the phase space mapping defined by equation (26). The matrix elements are obtained from MADGRAPH 4.4.26 [44].

For each parton multiplicity, we make a flat (uniform) scan over the relevant $n$-parton phase space using an implementation of the RAMBO algorithm [45] provided in VINCIA. In each phase space point, the tree-level expansion of the shower weight, $w_{\mathrm{PS}}$, is given by a sum over nested antenna functions, subjected to the selected ordering criterion. E.g., for $Z \rightarrow q g g \bar{q}$, the tree-level expansion of the shower weight is

$$
\begin{aligned}
w_{\mathrm{PS}}= & \left(\bar{a}_{g / q g}\left(q, g_{1}, g_{2}\right) \bar{a}_{g / q \bar{q}}\left(\widetilde{q g_{1}}, \widetilde{g_{1} g_{2}}, \bar{q}\right) \Theta\left(Q_{E}\left(\widetilde{q g_{1}}, \widetilde{g_{1} g_{2}}, \bar{q}\right)-Q_{E}\left(q, g_{1}, g_{2}\right)\right)+\right. \\
& \left.\bar{a}_{g / q g}\left(\bar{q}, g_{2}, g_{1}\right) \bar{a}_{g / q \bar{q}}\left(q, \widetilde{g_{1} g_{2}}, \widetilde{g_{2}} \bar{q}\right) \Theta\left(Q_{E}\left(\widetilde{q} \widetilde{g_{1} g_{2}}, \widetilde{g_{2}} \bar{q}\right)-Q_{E}\left(\bar{q}, g_{2}, g_{1}\right)\right)\right)\left|\mathcal{M}_{2}^{(0)}\right|^{2}
\end{aligned}
$$

where $Q_{E}$ denotes the evolution variable and tilded variables are obtained by reclustering the final-state momenta to intermediate 3-parton states, using the inverse of the shower kinematics map described in section 2.3. The $\Theta$ functions express the strong-ordering condition for each of the two possible clustering histories that lead from 2 to 4 partons in the shower. For an unordered shower, they would be absent (i.e., unity), whereas for a smoothly ordered shower, they would be replaced by the $P_{\text {imp }}$ factor defined in section 3.1. For higher numbers of partons, more terms are generated, for which we use an iterative code structure to compute the relevant sums.

The tree-level expansion of the shower weight $w_{\text {PS }}$ can then be compared to the norm squared of the appropriate fixed-order colour-ordered sub-amplitude squared, $\left|\mathcal{M}_{n}^{(0)}\right|^{2}$, forming the ratio

$$
R_{n}=\frac{w_{\mathrm{PS}}}{\left|\mathcal{M}_{n}^{(0)}\right|^{2}}
$$

This ratio thus represents an estimate of the relative accuracy of the shower (or, rather, its tree-level expansion) phase-space point by phase-space point. For simplicity, all couplings and colour factors are set to unity in this comparison.

By studying how the distribution of $R_{n}$ evolves with $n$, we obtain a useful indication of the accuracy of the shower, and how this accuracy evolves with parton multiplicity. We consider these comparisons to be fairly conservative, since the soft- and collinear-enhanced regions only occupy a relatively small corner of phase space in a flat scan. Similar comparisons to tree-level matrix elements were carried

\footnotetext{
${ }^{8}$ Subleading-colour properties were studied for the massless case in [6] and are not repeated here.
} 
out for massless showers in $[6,37,38]$. Here, we focus in particular on the modifications to these comparisons caused by non-vanishing masses.

For comparison purposes, we will plot the logarithm of such ratios, $\log _{10}\left(R_{n}\right)$, for different shower approximations, final state multiplicities, and parton masses. This logarithm gives a way of quantifying the amount of over or undercounting by the shower approximation. For phase space points for which the shower approximation reproduces the matrix element exactly, this logarithm is zero. For those points for which the logarithm is positive, the shower overestimates the matrix elements while it underestimate the matrix elements for negative values of this logarithm.

\section{1 $\mathrm{Z} \rightarrow \mathrm{Q} \overline{\mathrm{Q}}+$ gluons}

For massless quarks, the default $q \bar{q} \rightarrow q g \bar{q}$ dipole-antenna function, which describes the first $2 \rightarrow 3$ branching in the shower evolution, coincides with the matrix element for $Z \rightarrow q g \bar{q}$, and therefore the shower is "automatically" matched to the $Z \rightarrow 3$ matrix element. For massive quarks, however, the shower with default antennae differs slightly from the exact matrix element for $Z$ decay already at this order. This was discussed in sections 2.5 and 3.3, with an illustration provided in figure 5 .

Turning to $Z \rightarrow 4$ partons and more, in figure 6, we illustrate how quark masses affect the distribution of $R_{n}$, for $Z \rightarrow q \bar{q}+2$ gluons (left), +3 gluons (middle), and +4 gluons (right), for three different evolution criteria: no ordering (top), strong ordering in $p_{\perp}$ (middle), and smooth ordering in $p_{\perp}$ (bottom).

With no ordering (top row), the massless shower (solid black) has a large tail to the right, i.e., it substantially overcounts the matrix elements, as was also discussed in [6]. Towards the left of zero, it falls off extremely sharply (notice the logarithmic $y$ axis), implying that the unordered shower approximation is an almost strict overestimate of the matrix elements. The introduction of masses changes this picture drastically, with a moderate ratio $m_{Q} / E_{\mathrm{cm}}=0.1$ shown as a thin solid histogram and the larger $m_{Q} / E_{\mathrm{cm}}=0.3$ shown with dashes: for both mass values, a lot of the overcounting on the right of zero is removed, and for the larger ratio the regions below zero, where the shower underestimates the matrix-elements, is populated.

In the second row of figure 6 , the introduction of strong ordering in $p_{\perp}$ improves systematically on the non-ordered approximation and the introduction of quark masses does not spoil this improvement. Even for quite large quark masses, the distributions remain almost centred around $\log _{10}(R)=0$. However, as discussed in section 3.1, the price for strong ordering is the introduction of a dead zone, which we illustrate by plotting the underflow bin at $\log _{10}(R)=-2$. Its size corresponds to a few percent of the phase space volume.

In the last row, the change to a smooth ordering condition is illustrated. The dead zone is removed and, at least in the massless case, this smooth ordering condition further improves the agreement with the matrix elements relative to the strong-ordering case. It eliminates the tail of large undercounting that was present for $Z \rightarrow 5$ and 6 partons in the strong-ordering case and sharpens the peak around $\log _{10}(R)=0$ also for $Z \rightarrow 4$. This improvement is much less significant in the massive case, but since the dead zone is still removed, we use the smooth ordering option as our default choice for massive partons as well.

We note also that the effect of imposing strong ordering in $p_{\perp}$ is much more pronounced for massless quarks than for massive ones. To see this, we compare for instance the change in the black (massless) histogram between the top (unordered) and middle (strongly-ordered) left-hand panes of figure 6 . We 


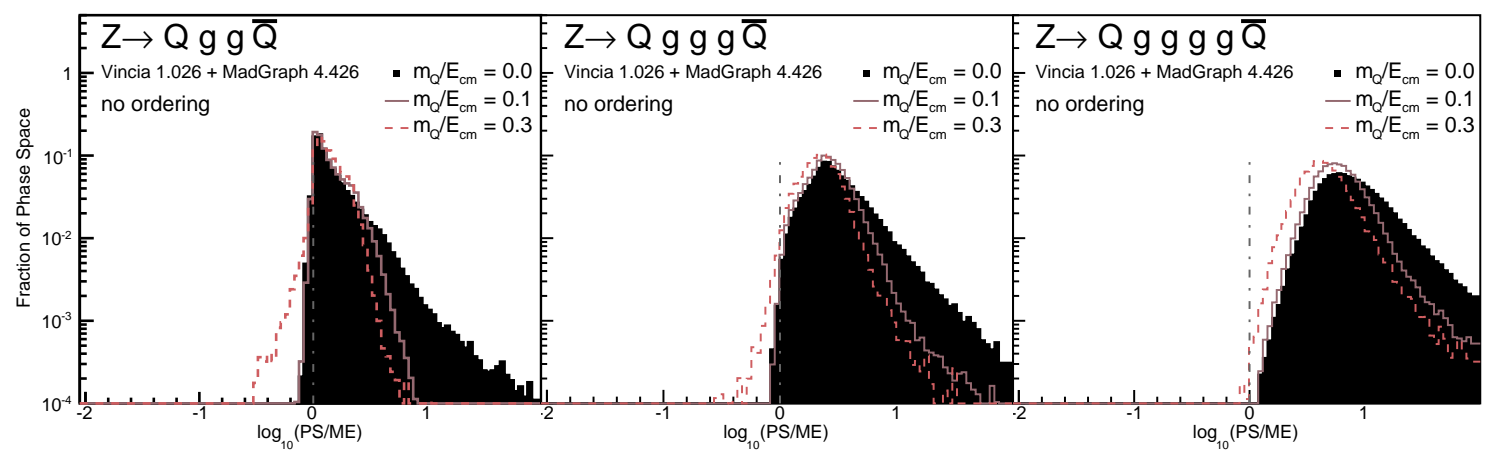

(a) no ordering

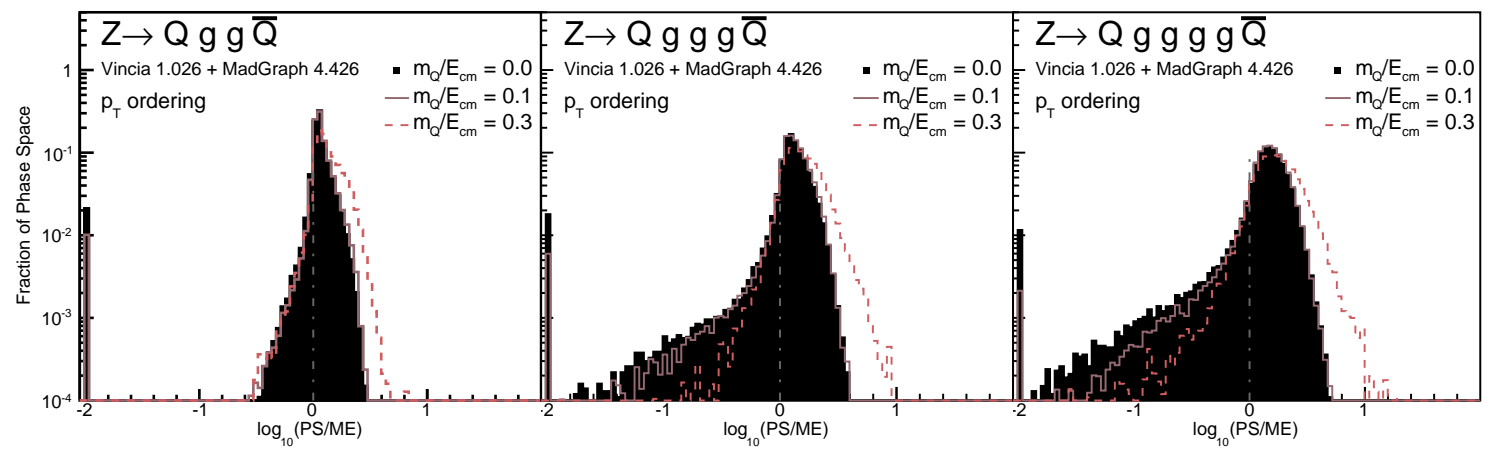

(b) strong ordering in $p_{\perp}$

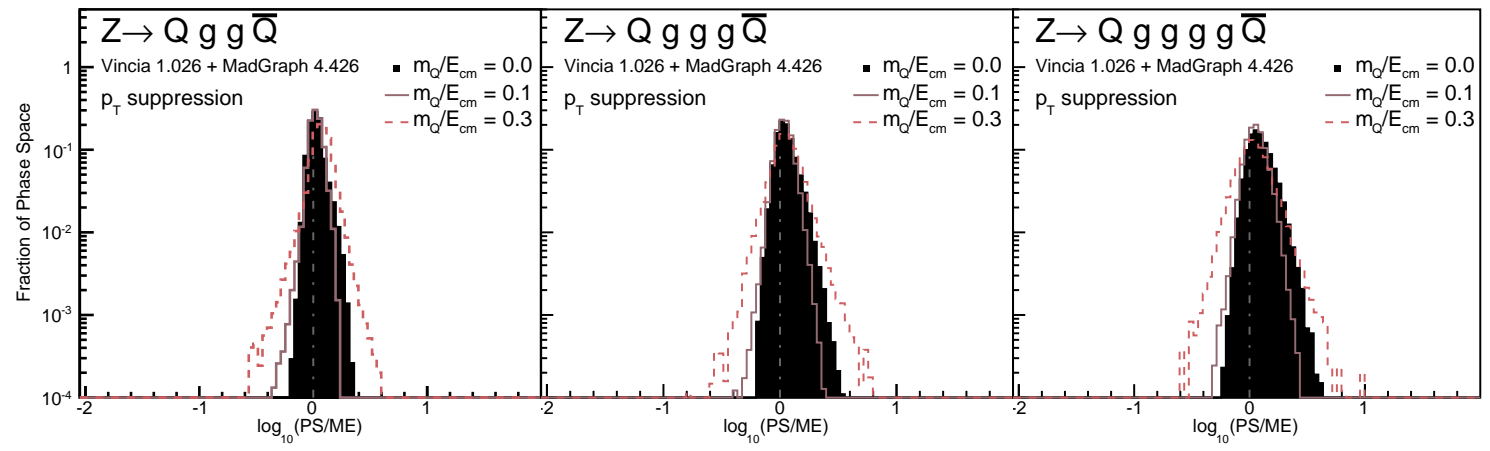

(c) smooth suppression of emissions not ordered in $p_{\perp}$

Figure 6: Histograms of $\log _{10}\left(R_{n}\right)$, as defined in the text, in a flat phase space scan, for $n=4$ (left panes), $n=5$ (middle panes), and $n=6$ (right panes), for not ordered (top row), strongly ordered (middle row), and smoothly ordered (bottom row) shower approximations. The parton shower uses the default dipole-antenna set defined in section 3 . 

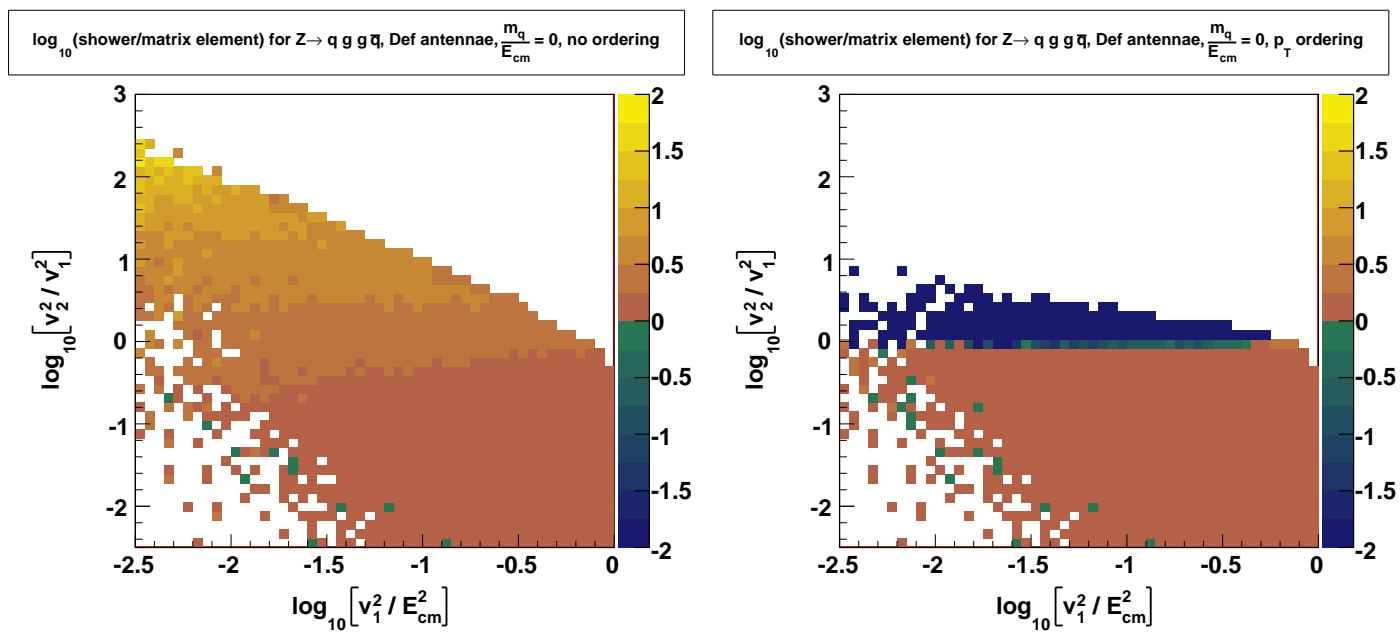

Figure 7: $\log _{10}\left(R_{4}\right)$ for $Z \rightarrow q g g \bar{q}$, for $m_{q}=0$, as a function of $x=\log _{10}\left(p_{\perp, 1}^{2} / E_{\mathrm{cm}}^{2}\right)$ and $y=$ $\log _{10}\left(p_{\perp, 2}^{2} / p_{\perp, 1}^{2}\right)$. Left pane: no ordering. Right pane: strong ordering in $p_{\perp}$.

investigate this further in the 2D phase-space plots presented in figures 7 and 8 .

Figure 7 shows the case for massless quarks. In the left-hand pane, no ordering condition is imposed; in the right-hand pane, strong ordering in $p_{\perp}$. The axes of the figure have been chosen to be logarithmic in the two successive branching scales $p_{\perp, 1} / E_{\mathrm{cm}}$ and $p_{\perp, 2} / p_{\perp, 1}$, respectively, with $p_{\perp, 1}$ the emission scale of the first branching and $p_{\perp, 2}$ the emission scale of the second branching. Among the two shower histories for $Z \rightarrow q g g \bar{q}$, we show the $p_{\perp}$ values of the larger contribution on the plot. An average over the phase space points compatible with the corresponding values on the $x$ - and $y$-axes is shown, obtained using the same flat scans of the phase space as for the one-dimensional phase space plots above.

The effect of the strong-ordering condition is clearly visible in the right-hand pane of figure 7 , removing the shower contributions in the upper half of the plot, corresponding to unordered branchings.

Comparing the left-hand pane in figure 7 (massless case, no ordering) to that in figure 8 (massive case, with $m / E_{\mathrm{cm}}=0.3$, no ordering), one notices that the part of the phase space in which the overestimate of the shower is biggest in the massless case corresponding to the region where the second emission is at a higher scale than the first one, is almost inaccessible in the massive case due to kinematic restrictions.

In accordance with this, the effect of imposing strong ordering in $p_{\perp}$ is comparably small for heavy quarks, as shown in the right-hand pane of figure 8. We conclude that to impose an ordering condition is much more important for massless quarks than it is for massive ones.

\subsection{Including massless $\mathrm{g} \rightarrow \overline{\mathbf{q}}^{\prime} \mathrm{q}^{\prime}$ splittings}

The gluon-splitting $g \rightarrow q \bar{q}$ dipole-antenna functions $\left(a_{\bar{q}^{\prime} / q g}\right.$ and $a_{\bar{q} / g g}$ in the notation adopted here) only contain single poles in the region where the secondary quark-antiquark pair becomes collinear. Those antennae are therefore less singular than their gluon-emission counterparts, and hence there are intrinsically fewer $g \rightarrow q \bar{q}$ splittings than gluon emissions occurring (independently of the difference $C_{A}$ 

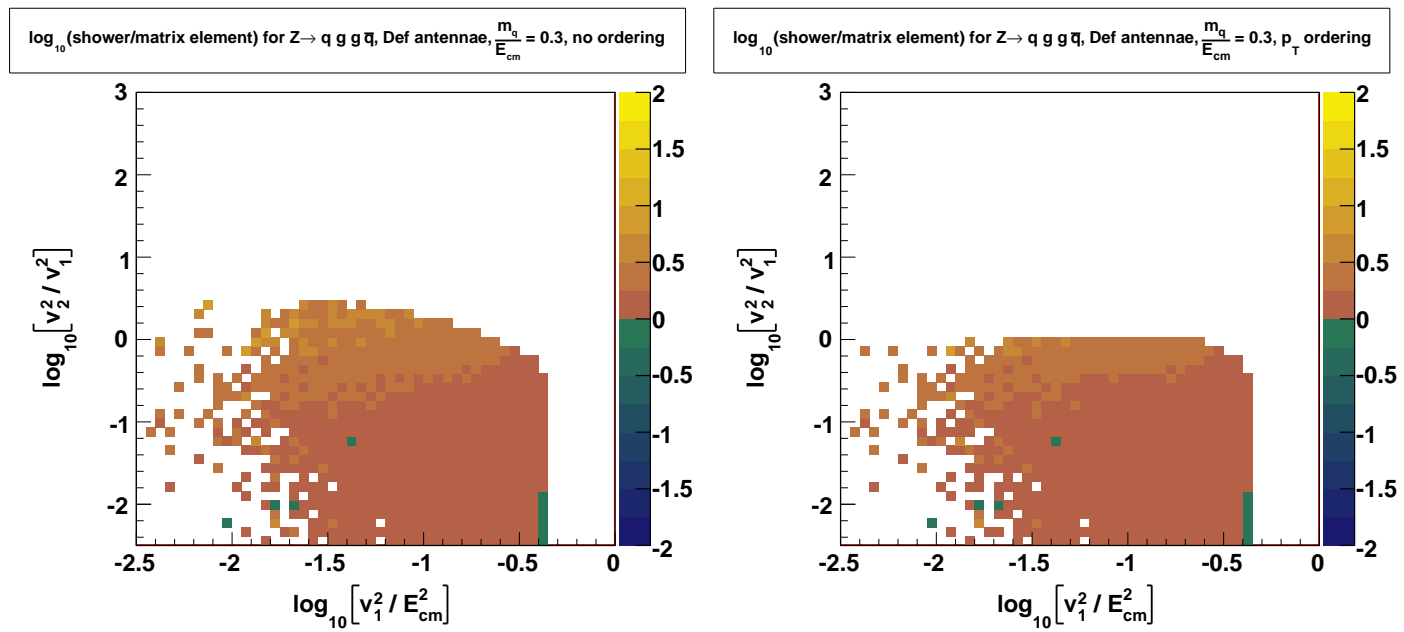

Figure 8: $\log _{10}\left(R_{4}\right)$ for $Z \rightarrow q g g \bar{q}$, for $m_{q} / E_{\mathrm{cm}}=0.3$, as a function of $x=\log _{10}\left(p_{\perp, 1}^{2} / E_{\mathrm{cm}}^{2}\right)$ and $y=$ $\log _{10}\left(p_{\perp, 2}^{2} / p_{\perp, 1}^{2}\right)$. Left pane: no ordering. Right pane: strong ordering in $p_{\perp}$.

vs $n_{f} \widehat{T}_{R}$ in colour/flavour factors). However, for those gluon splittings that $d o$ occur, the smaller relative size of the (universal) singular terms, on which the shower approximations are based, as compared to possible non-singular (and non-universal) terms, imply that one can expect an overall worsening of the shower approximation for processes involving $g \rightarrow q \bar{q}$, as compared to ones involving only gluon emission. An immediate consequence of this is, for instance, that the amount of strange and heavier quarks produced in perturbative $g \rightarrow q \bar{q}$ splittings is associated with substantial uncertainties in all current shower models.

A first illustration of this feature is given by figure 9 which shows the ratio of the expanded parton shower (with no ordering condition imposed) to the tree-level matrix element for $Z \rightarrow Q \bar{q}^{\prime} q^{\prime} \bar{Q}$. The $x$ axis now ranges from -4 to 4 , rather than -2 to 2 , allowing for a much larger range of shower-to-matrixelement ratios. This accommodates the most important feature in figure 9: the tail of high overestimates of the unordered shower approximation for a massless primary quark-antiquark pair has $R$ values extending up to approximately $10^{4}$ as opposed to approximately $10^{2}$ for gluon emission, for the same $y$ range (i.e., same fraction of flat phase space).

A phase-space scan similar to the ones shown in figures 7 and 8, revealed that most of the very high overestimates occur for configurations where the secondary quark-antiquark pair takes up almost all of the energy, which in turn forces the primary quarks to be soft. One possible shower history leading to such a particular configuration is obtained by having a collinear high- $z$ gluon emission followed by a very hard $g \rightarrow q \bar{q}$ splitting, or represented pictorially illustrated in figure 10 . Such occurrences are apparently all too frequent, in the unordered shower. Physically, we interpret this as a screening effect which is missing in the unordered approximation. By independently adding the splitting probabilities in each of the $q g$ and $g \bar{q}$ antennae, we are not taking into account any screening effects produced by the collective $q g \bar{q}$ system, which become particularly relevant when two or more of those partons are collinear with respect to each other and hence should maximally screen each other.

As illustrated by the histograms for light primary quarks (thin solid line) and for heavy primary 


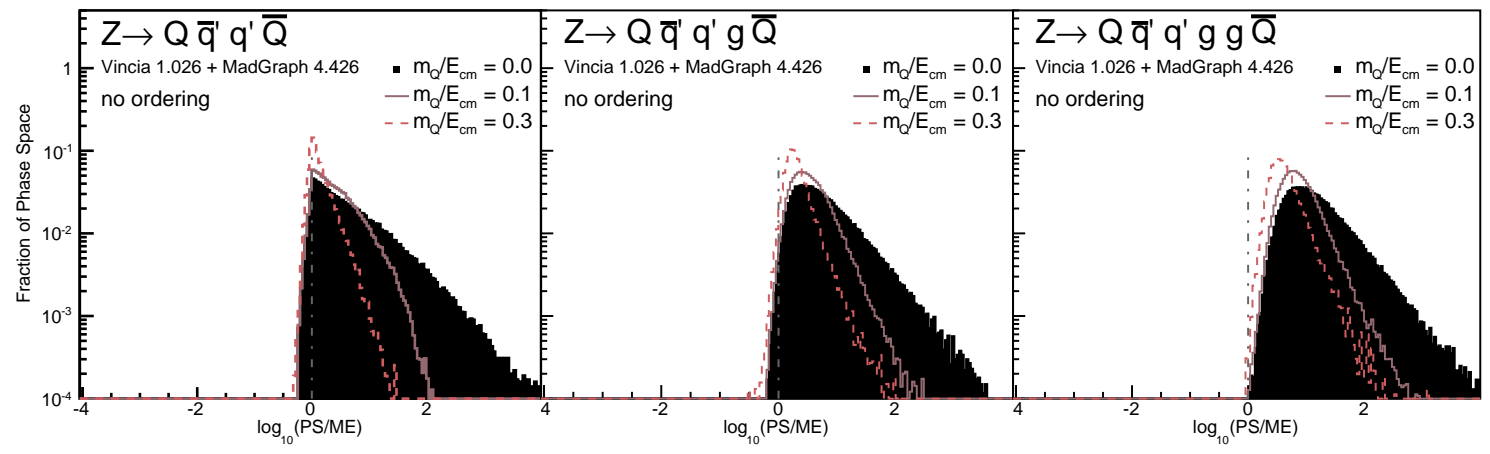

Figure 9: Histograms of $\log _{10}\left(R_{n}\right)$, as defined in the text, for $Z \rightarrow Q \bar{q}^{\prime} q^{\prime}(g g) \bar{Q}$ in a flat phase space scan, for $n=4$ (left pane), $n=5$ (middle pane), and $n=6$ (right pane). No ordering condition is imposed.

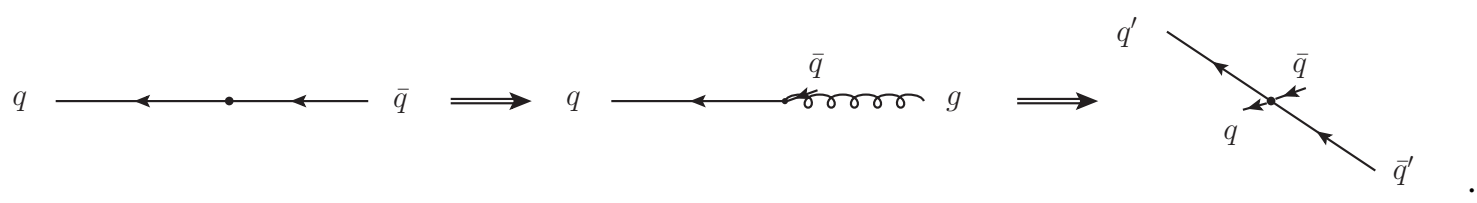

Figure 10: A high- $z$ gluon emission followed by a hard $g \rightarrow q \bar{q}$ splitting.

quarks (dashed) in figure 9, the introduction of non-zero masses for the primary quarks improves this situation, since the configurations where the shower overestimates are the largest in the massless case simply cannot be reached for heavy primary quarks due to kinematical constraints. In terms of coherence, the strong dampening of the collinear singularity for massive emitters leads to an absence of the subsequent very strong coherence dampening that is present in the massless case.

We now turn to ordered showers. By analogy with the case for gluon emission, figure 6, we expect that we can get rid of a significant part of the high shower overestimates for highly energetic secondary quark-antiquark pairs by imposing a strong ordering condition in the secondary quark-antiquark mass $m_{q \bar{q}}$ for gluon splittings. An alternative choice that cannot a priori be excluded would be to use $p_{\perp-}$ ordering for gluon splitting as well. In figure 11, we make a first comparison of these two possibilities, for processes with primary massless $q \bar{q}$ parent partons and involving gluon splittings in a second branching step. Either we use strong ordering in $Q_{E}=2 p_{\perp}$ for gluon emission and ordering in $Q_{E}=m_{q \bar{q}}$ for gluon splitting (shown in the left-hand pane of the figure), or we use strong ordering in $Q_{E}=2 p_{\perp}$ for both branching processes (shown in the right-hand pane). Specifically, in the left-hand pane, the subsequent gluon splitting is vetoed if $m_{q^{\prime} \bar{q}^{\prime}}>2 p_{\perp}(q, g, \bar{q})$, while on the right-hand pane, the gluon splitting is vetoed if $p_{\perp}\left(q, \bar{q}^{\prime}, q^{\prime}\right)>p_{\perp}(q, g, \bar{q})$ or $p_{\perp}\left(\bar{q}, q^{\prime}, \bar{q}^{\prime}\right)>p_{\perp}(q, g, \bar{q})$ respectively. A substantial over-counting for highly energetic secondary quark-antiquark pairs remains in the $p_{\perp}$-ordered case (right-hand pane, top right corner), while an undercounting results when the evolution variable is changed to $m_{q \bar{q}}$ in the second branching step (left). We conclude that the strong ordering in $m_{q \bar{q}}$ does a better job of suppressing the high overestimates for the regions of the phase space where there is no leading-log contribution (top right of the plots).

As in the case of gluon emission, we wish to avoid dead zones by switching to a smooth suppression of gluon splittings, using the suppression factor $P_{\text {imp }}$, defined in equation (51). However, in figure 12, 


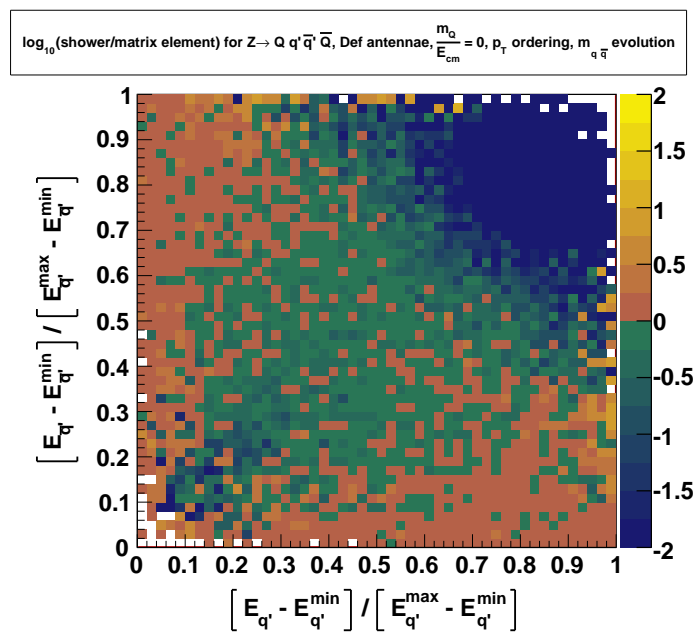

(a) Evolution variable $m_{q \bar{q}}$ for gluon splitting.

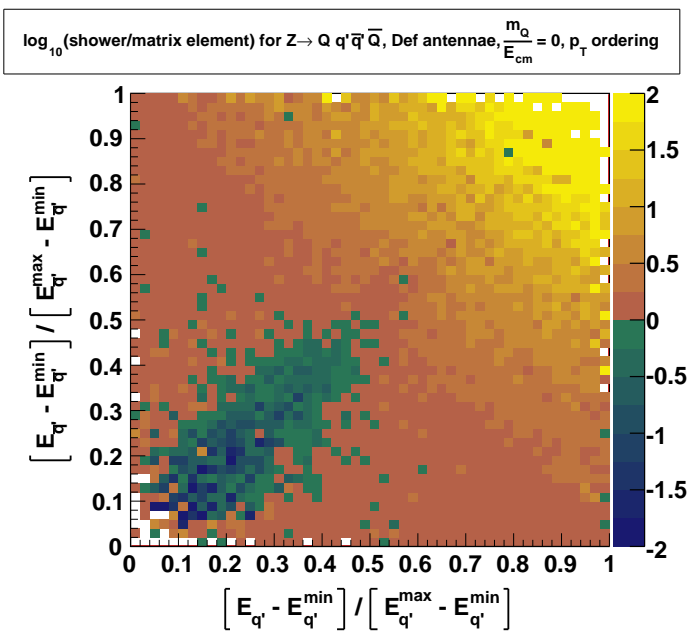

(b) Evolution variable $p_{\perp}$ for gluon splitting

Figure 11: $\log _{10}\left(w_{\mathrm{PS}} /|\mathcal{M}|^{2}\right)$ for $Z \rightarrow q \bar{q}^{\prime} q^{\prime} \bar{q}$ as a function of the secondary quark energies, rescaled to range from 0 to 1. Left: Evolution variable $m_{q \bar{q}}$ for gluon splitting, resulting in the strong ordering condition $m_{q^{\prime} \bar{q}^{\prime}}<2 p_{\perp}^{q \bar{q} \rightarrow q g \bar{q}}$. Right: Evolution variable $p_{\perp}$ for gluon splittings, resulting in the strong ordering condition $p_{\perp}^{q g \rightarrow q \bar{q}^{\prime} q^{\prime}}<p_{\perp}^{q \bar{q} \rightarrow q g \bar{q}}$. All quarks are massless.

we illustrate that in this gluon splitting case, a naive application of this suppression factor $P_{\text {imp }}$, results in an overcounting for both choices of gluon-splitting variables. This figure shows the same distributions as in figure 11, but with the strong-ordering condition replaced by a smooth one.

In a dipole-antenna shower that employs ordering in $p_{\perp}$ for all branchings, or for our smoothordering shower variant, an additional suppression mechanism is therefore needed to remove this overcounting and get reasonable agreement between approximated shower vs matrix elements for processes involving gluon splittings. The Lund dipole cascade implemented in the ARIADNE program [8] uses the following factor to modify its gluon splitting antenna functions,

$$
P_{\text {Ari }}=\frac{2 m_{N}^{2}}{m_{I K}^{2}+m_{N}^{2}}
$$

where $m_{I K}^{2}$ is the invariant mass squared of the parent antenna-dipole and $m_{N}^{2}$ is that of the neighbouring dipole-antenna. Thus, if the preceding branching was collinear, with $m_{N}^{2} \rightarrow 0$, this factor produces a very strong suppression, while if the two dipole-antennae that share the splitting gluon have exactly equal sizes it goes to unity. ${ }^{9}$ In figure 13 , we show that the use of the $P_{\text {Ari }}$ factor suppresses the overestimates visible in figure 12 to a large degree, with a slightly better agreement obtained in the left-hand pane (for interleaved $p_{\perp}$ and $m_{q \bar{q}}$ evolution) than in the right-hand one (with all processes ordered in $\left.p_{\perp}\right)$.

The expansion of the resulting weights for $Z \rightarrow 4,5$, and 6 partons with smooth ordering in $2 p_{\perp}$ for gluon emissions and in $m_{q \bar{q}}$ for gluon splittings, are shown in figure 14a and for smooth ordering in $p_{\perp}$

\footnotetext{
${ }^{9}$ If the neighbouring dipole-antenna is much larger than the parent antenna, it even produces a slight enhancement, by up to a factor of 2, but this is more than compensated for by the reduction in the splitting probability of the neighbour itself.
} 


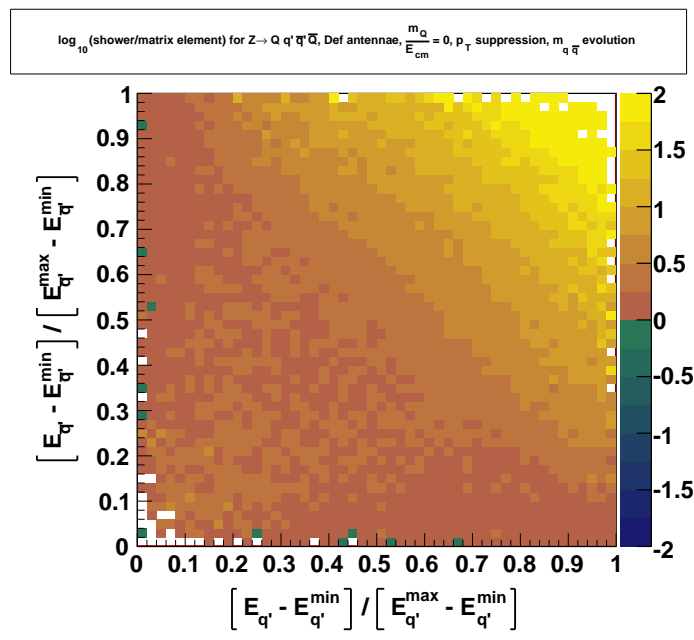

(a) Evolution variable $m_{q \bar{q}}$ for gluon splitting.

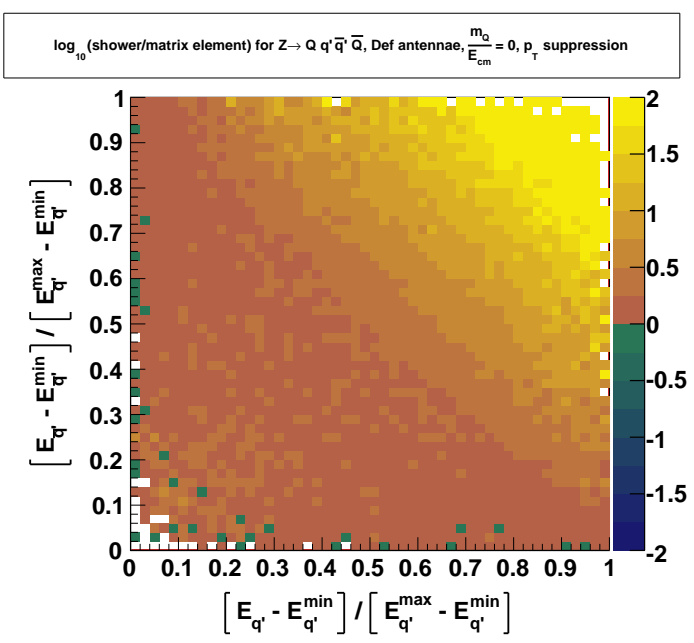

(b) Evolution variable $p_{\perp}$ for gluon splitting

Figure 12: $\log _{10}\left(w_{\mathrm{PS}} /|\mathcal{M}|^{2}\right)$ for $Z \rightarrow q \bar{q}^{\prime} q^{\prime} \bar{q}$ as a function of the secondary quark energies, rescaled to range from 0 to 1. All quarks are massless, smooth ordering is imposed, $p_{\perp}$ is used as the evolution variable for gluon emission. Left: Use $m_{q \bar{q}}$ for gluon splitting. Right: Use $p_{\perp}$ for gluon splitting.

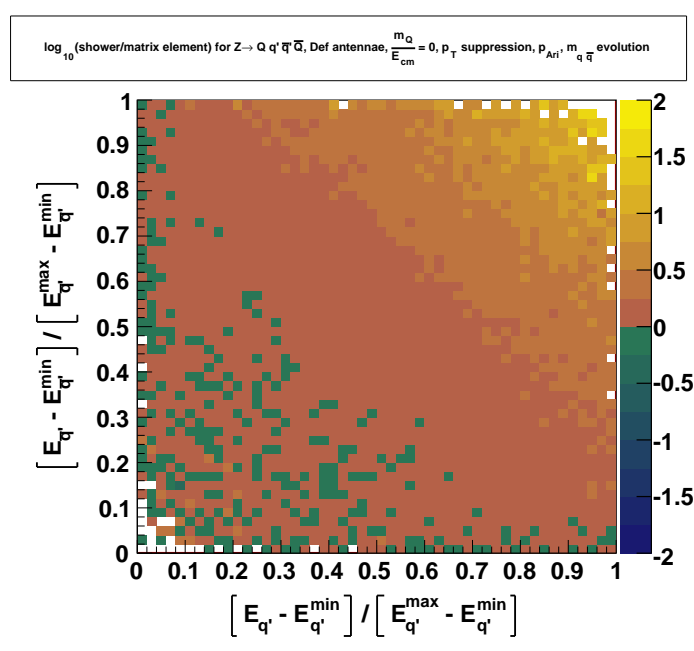

(a) Evolution variable $m_{q \bar{q}}$ for gluon splitting.

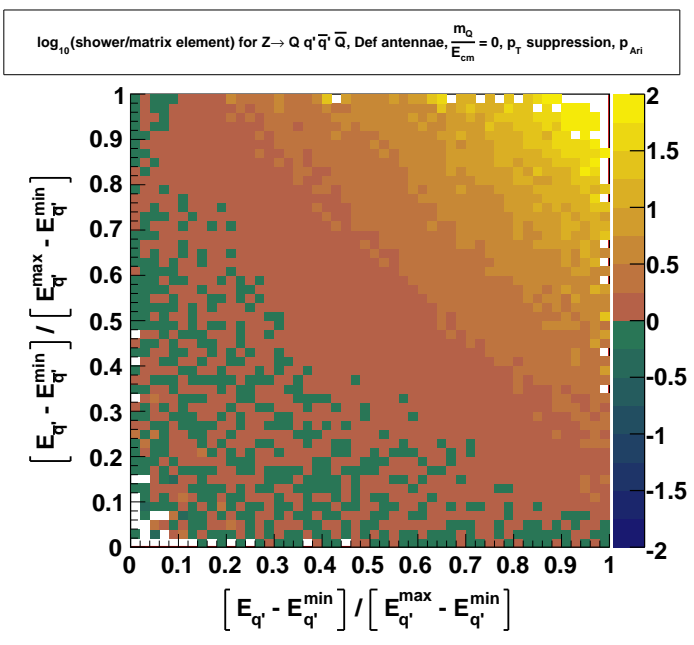

(b) Evolution variable $p_{\perp}$ for gluon splitting

Figure 13: $\log _{10}\left(R_{4}\right)$ for $Z \rightarrow q \bar{q}^{\prime} q^{\prime} \bar{q}$ as a function of the secondary quark energies, rescaled to range from 0 to 1. Smooth suppression of unordered emissions, with $P_{\text {Ari }}$ factor.

for all branching processes in figure $14 \mathrm{~b}$.

Although still far from the very good agreement obtained in the pure gluon-emission case (as compared with figure $6 \mathrm{c}$ ), both the centre and the width of the weight distributions shown in figure $14 \mathrm{~b}$ where smooth ordering including this additional suppression factor $P_{\text {Ari }}$ is imposed for secondary massless $q \bar{q}$ 


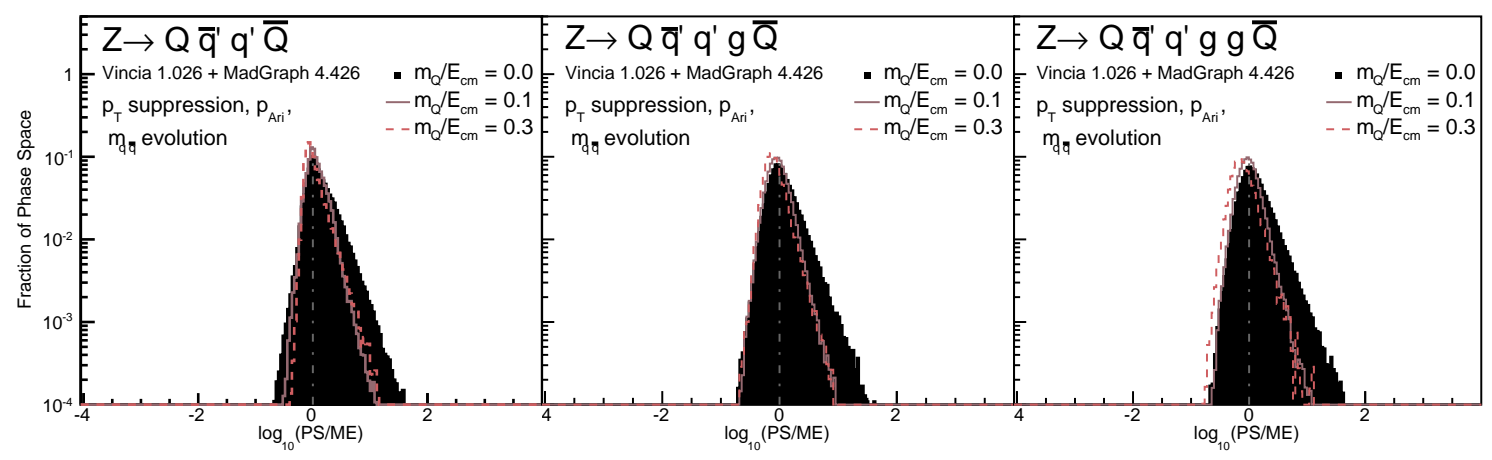

(a) Using $p_{\perp}$ for gluon emissions and $m_{q \bar{q}}$ for secondary $q \bar{q}$ production.

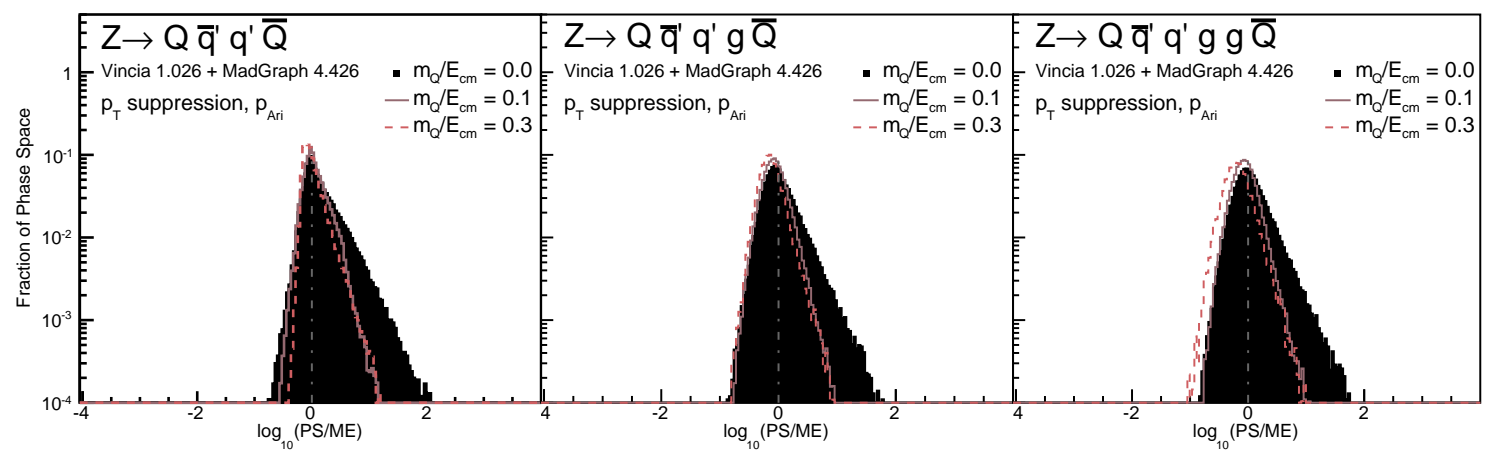

(b) Using $p_{\perp}$ for both gluon emissions and for secondary $q \bar{q}$ production.

Figure 14: Histograms of $\log _{10}\left(R_{n}\right)$, for $Z$ decay into a massive primary quark pair, a massless secondary quark pair and up to two gluons in a flat phase space scan, for $n=4$ (left panes), $n=5$ (middle panes), and $n=6$ (right panes). The $P_{\text {Ari }}$ factor is used for gluon splittings. Top row: smooth ordering using $p_{\perp}$ as the scale for gluon emission and $m_{q \bar{q}}$ for gluon splittings. Bottom row: smooth ordering using $p_{\perp}$ for both gluon emissions and gluon splittings.

production, are now in tolerable agreement with the leading order (LO) matrix elements over a substantial fraction of phase space. Matching to the LO matrix elements can obviously be used to improve this agreement further, up to the orders for which matrix elements are available (see section 3.5).

It should be emphasized, however, that the centre position of the distribution $w_{\mathrm{PS}} /|\mathcal{M}|^{2}$ is highly sensitive to the finite parts of $a_{\bar{q}^{\prime} / q g}$. Since these pieces of $a_{\bar{q}^{\prime} / q g}$ are not universal, the fact that the most frequent ratio of $w_{\mathrm{PS}} /|\mathcal{M}|^{2}$ is almost unity as demonstrated in figure 14 a cannot be expected to be universal for all processes involving gluon splitting either. This is an unavoidable consequence of the less pronounced singular behaviour for these antennae, as compared to the gluon-emission ones.

\subsection{Including massive $\mathrm{g} \rightarrow \overline{\mathrm{Q}}^{\prime} \mathrm{Q}^{\prime}$ splittings}

As a final set of comparisons we include massive $g \rightarrow \bar{Q} Q$ splittings in the shower expansion. Since the corresponding dipole-antenna functions do not contain any poles at all (though one does appear for $m_{Q} \rightarrow 0$ ), we should expect the shower approximation to be at its worst for this case, translating to very large uncertainties on, e.g., the amount of $g \rightarrow b \bar{b}$ splittings produced by it. 


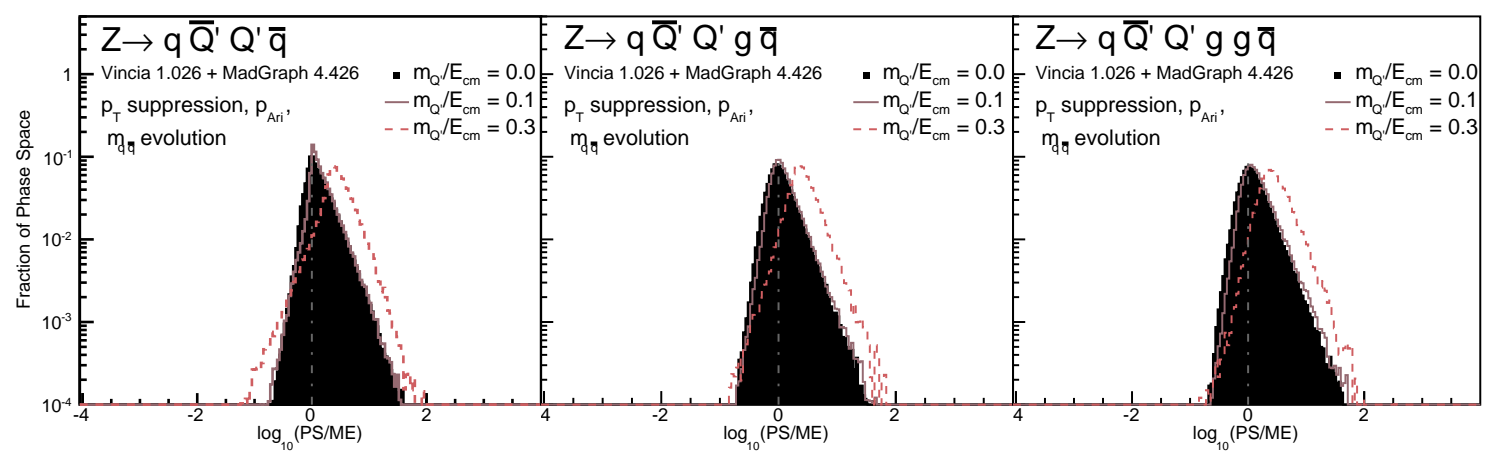

(a) Using $p_{\perp}$ for gluon emissions and $m_{q \bar{q}}$ for gluon splittings.

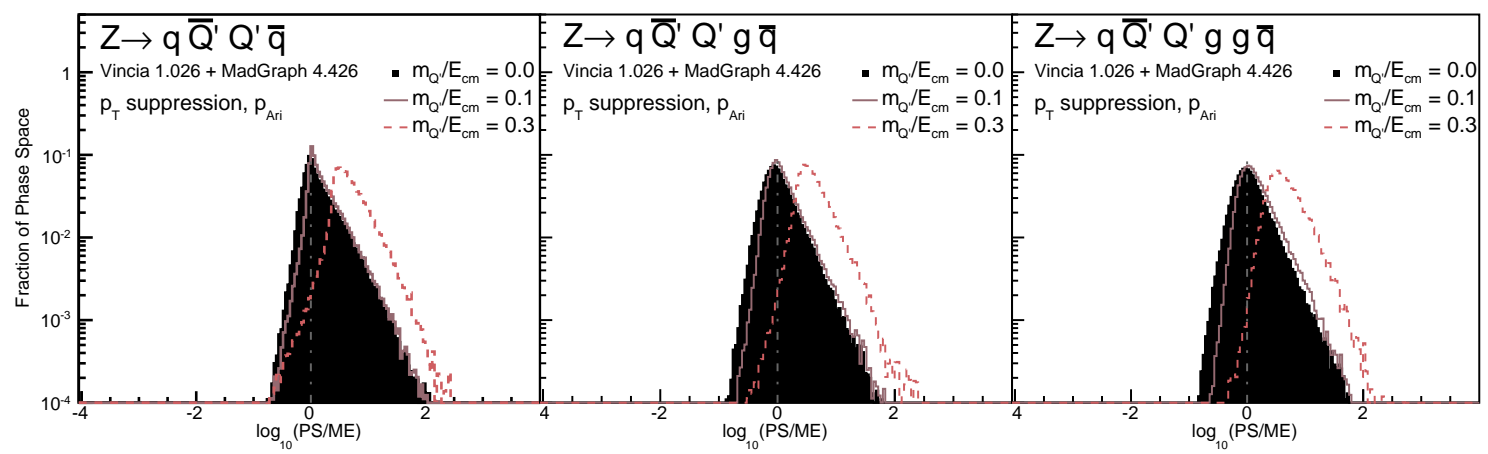

(b) Using $p_{\perp}$ for gluon emissions and gluon splittings.

Figure 15: Histograms of $\log _{10}\left(R_{n}\right)$, as defined in the text, for $Z$ decay into a massless primary quark-antiquark pair, a massive secondary quark-antiquark pair and up to two gluons in a flat phase space scan, for $n=4$ (left panes), $n=5$ (middle panes), and $n=6$ (right panes). Unordered emissions are suppressed smoothly, for gluon splittings $P_{\text {Ari }}$ is used. In the top row, the suppression factor for unordered branchings is calculated using $p_{\perp}$ as the scale for gluon emission and $m_{q \bar{q}}$ as the scale for gluon splittings. In the bottom row, the suppression factor is calculated using $p_{\perp}$ for both gluon emissions and gluon splittings. Note: the parton shower uses the default antenna set.

However, as illustrated by the plots in figure 15, the agreement is in fact at the same level as that obtained for massive parents in the previous subsection, except for a slight tilt of the distribution for rather heavy secondary quarks. We note that this is especially true for the "interleaved evolution" choice of using $Q_{E}=2 p_{\perp}$ for gluon emissions and $Q_{E}=m_{q \bar{q}}$ for gluon splittings, cf. figure 15a, as compared to using $p_{\perp}$ for all branchings as illustrated in figure $15 \mathrm{~b}$.

This strengthens our motivation for using the interleaved $p_{\perp}$ - and mass-ordered evolution as the default in VINCIA. Note also that we have checked that this conclusion appears to be robust against at least moderate variations of the antenna function finite terms. We conclude that there is still significant uncertainties surrounding massive $g \rightarrow Q \bar{Q}$ splittings, but that the default choices made in VINCIA can at least be considered a sensible starting point. Of course, matching to matrix elements can still improve the situation, in particular for secondary quark-antiquark pairs of high invariant mass, by increasing the multiplicity at which the arbitrary finite parts of the antenna functions start to matter. 


\section{Comparison to Analytic Resummation}

Observables involving massive particles, like heavy-quark fragmentation processes, can be considered as being collinear-safe since collinear divergencies are regulated by the finite value of the heavy quark mass $m$. Thus, such processes can be computed order by order in perturbation theory. Nevertheless, as mentioned in section 2.4, mass-dependent logarithms of the form $\ln \left(Q^{2} / m^{2}\right)$, where $Q$ is the typical scale of the hard scattering process, appear at each order in perturbation theory. When the hard scale $Q$ is much larger than $m$, these quasi-collinear logarithmic contributions can be large and have to be resummed to all orders to obtain reliable predictions for these observables.

This resummation can be performed analytically, in a process-independent way, by using the perturbative fragmentation formalism [46,47], which is summarized briefly below. Alternatively it can be performed numerically, using a parton-shower, such as VINCIA.

In this section, our aim is to verify on a particular example, that the VINCIA implementation developed in this paper, based on the exponentiation of massive dipole-antenna functions which reproduce the soft and the quasi-collinear limit of tree-level matrix-elements, performs the resummation of quasicollinear logarithms correctly. We do this by comparing the predictions of VINCIA with those obtained from the analytic calculation of [33] for the inclusive production of a single heavy meson $\mathrm{H}$ in $e^{+} e^{-}$ collisions.

The single inclusive production of a heavy meson $H$ as obtained by the process $e^{+} e^{-} \rightarrow V \rightarrow$ $H(p)+X$ can be described by the production of a heavy quark pair $Q-\bar{Q}$ which subsequently hadronize to yield a heavy meson $H$. This hadronization process can be described by non-perturbative (heavy quark)-to-hadron fragmentation function (such as for example in the model of Peterson [48]) whose free parameters have to be extracted from the data.

Since we are mainly interested in the perturbative contributions to the inclusive cross section, we shall here ignore this non-perturbative contribution. Consequently, we compare the predictions obtained from the calculation of the inclusive production of a heavy quark pair with our predictions obtained with VINCIA without hadronization.

We consider the inclusive production of a heavy quark pair in $e^{+} e^{-}$collisions in the kinematical region where the centre-of mass energy $E_{\mathrm{cm}}$ of the collisions is much larger than the heavy quark mass $m$, i.e. $E_{\mathrm{cm}} \gg m$. At the same time the heavy quarks are produced in the perturbative regime with a mass $m$ which is large enough so that $\left(m \gg \Lambda_{\mathrm{QCD}}\right)$. We consider the following distribution,

$$
\mathcal{D}\left(x, E_{\mathrm{cm}}^{2}, m^{2}\right) \equiv \frac{1}{\sigma_{\mathrm{tot}}} \frac{\mathrm{d} \sigma}{\mathrm{d} x}
$$

with $\sigma_{\text {tot }}$ the total hadronic cross section in $e^{+} e^{-}$. The energy fraction $x$ of the heavy quark system with momentum $p$ is given by $x=2 p \cdot\left(p_{e^{+}}+p_{e^{-}}\right) / E_{\mathrm{cm}}^{2}$.

Since we are mainly interested in the large $x$ behaviour of the cross section, we concentrate on its so-called flavour-non-singlet contribution. In this case, the ingredients to the cross section are dependent on the difference of two flavour-non-identical parton species.

In the following we shall first recall the main ingredients of the analytic calculation of [33] before presenting our comparison. 


\subsection{The heavy quark fragmentation formalism}

The inclusive production of heavy quarks in $e^{+} e^{-}$annihilation is a purely perturbative process which can be described by the perturbative fragmentation formalism in which collinearly-enhanced contributions are resummed. In the large $x$ region, logarithmically enhanced contributions due to soft radiation can also occur. A reliable theoretical prediction for this heavy quark production process can therefore only be obtained if both class of logarithms are appropriately resummed. This combined resummation has been performed in [33] up to the next-to-leading logarithmic (NLL) accuracy. In the following we shall first describe how the collinear-enhanced logarithms are resummed before including soft-gluon resummation effects as well.

Thanks to the factorization theorem of mass singularities the cross section for the production of a hadron $H$ in $e^{+} e^{-}$collisions can be written as a convolution of a process-dependent coefficient function $C_{(a)}^{e^{+} e^{-}}$and a parton-to-hadron fragmentation function denoted by $D_{a / H}$, for each parton $a=(q, \bar{q}, g)$ involved, treating all flavours as massless. Performing a power series expansion in $\alpha_{s}$, one can compute each of the coefficient functions $C_{(a)}^{e^{+} e^{-}}$as a massless QCD partonic cross section and the $\mu_{F}^{2}$ evolution of $D_{a / H}$, within perturbation theory. The $\mu_{F}^{2}$ evolution of $D_{a / H}$ is performed using the well-known Altarelli-Parisi evolution equations [34,49] which enable the resummation of logarithmic contributions of collinear origin in $D_{a / H}$. Furthermore, provided $D_{a / H}$ is known at some initial factorization scale called $\mu_{0 F, H}$, its form at any other higher factorization scale $\mu_{F, H}$ can be determined from this evolution equation. Its form at the initial scale $\mu_{0 F, H}$, chosen such that $\mu_{0 F, H} \sim \Lambda_{\mathrm{QCD}}$, is a purely nonperturbative contribution which has either to be modelled phenomenologically and/or determined from data.

Within the framework of the perturbative fragmentation formalism, the cross section $\sigma_{Q}$ for the inclusive production in $e^{+} e^{-}$collisions, of a heavy quark pair $Q-\bar{Q}$ is given as a generalization of the fragmentation formalism for hadrons described above, as follows: For each parton $a=q, \bar{q}, g$, (still treating all flavours as massless), and for a given type of heavy quark $Q$ (or $\bar{Q}$ ), the heavy quark production cross section $\sigma_{Q}$ is given as a convolution of the previously defined coefficient function $C_{(a)}^{e^{+} e^{-}}$with $D_{a / Q}$, the perturbative fragmentation function of the massless parton $a$ into $Q$. In the most general case, all massless partonic contributions (including gluonic ones) to coefficient functions and fragmentation functions have to be taken into account. As we restrict ourselves in this section to the non-singlet part of the cross section, only the primary production of a quark-antiquark pair of the given type will contribute, secondary production will be neglected.

In calculations involving all-order resummation, it is usual to consider the equivalent expression in Mellin moment space where one uses the customary definition of the Mellin transform

$$
f_{N}=\int_{0}^{1} \mathrm{~d} x x^{N-1} f(x)
$$

which transforms the convolution in $x$-space into a product in Mellin space. Using the perturbative fragmentation formalism as described above, the $N$ moments of the inclusive distribution $\mathcal{D}$ may be written as,

$$
\mathcal{D}\left(E_{\mathrm{cm}}^{2}, m^{2}\right)=\frac{\sigma^{(L O)}}{\sigma_{\mathrm{tot}}} C_{N}^{\left(e^{+} e^{-}\right)}\left(\alpha_{S}\left(\mu^{2}\right), E_{\mathrm{cm}}^{2}, \mu^{2}, \mu_{F}^{2}\right) D_{N}\left(\mu_{F}^{2}, m^{2}\right)
$$

where $\sigma_{\text {tot }} / \sigma^{(L O)}$ is given at order $\alpha_{s}$ by $\left(1+\alpha_{s} / \pi\right) . C_{N}^{\left(e^{+} e^{-}\right)}$denotes the Mellin transform of the $e^{+} e^{-}$ coefficient function while $D_{N}$ stands for the Mellin moments of the flavour non-singlet component of 
the perturbative fragmentation function $D(x)$, as defined in equation (77). Both of these contributions depend on the factorization scale $\mu_{F}$ which was introduced by factorizing the cross section.

As mentioned before, we concentrate on the large- $x$ behaviour, i.e $x \rightarrow 1$ (in momentum space) or equivalently, in Mellin space on the large- $N$ limit $(N \gg 1)$ of this cross section. Note that, in [33] the inversion of the results to $x$ space is performed using the Minimal Prescription of [50,51], but we shall not use those results in what follows. We shall in fact compare the results obtained with VINCIA to the predictions of the analytic calculation obtained only in Mellin space, using equation (78).

The resummation of collinear-enhanced logarithms of $E_{\mathrm{cm}}^{2} / \mathrm{m}^{2}$ is achieved in equation (78) by writing the $\mathrm{N}$ moments of the perturbative fragmentation function, denoted by $D_{N}$ in this equation, as the product of an evolution operator $E_{N}$ and $D_{N}^{\text {ini }}$, the perturbative initial condition for the heavy quark fragmentation function. Both of these functions depend on the factorization scale $\mu_{F}$ and on the starting point of the evolution called $\mu_{0 F}$. The perturbative fragmentation function in Mellin space denoted by $D_{N}$ reads,

$$
D_{N}\left(\mu_{F}^{2}, m^{2}\right)=E_{N}\left(\mu_{F}^{2}, \mu_{0 F}^{2}\right) D_{N}^{\mathrm{ini}}\left(\alpha_{S}\left(\mu_{0}^{2}\right), \mu_{0}^{2}, \mu_{0 F}^{2}, m^{2}\right) .
$$

The evolution operator $E_{N}$ is the solution of the Altarelli-Parisi evolution equations written in Mellin space as,

$$
\frac{\mathrm{d} E_{N}\left(\mu_{F}^{2}, \mu_{0 F}^{2}\right)}{\mathrm{d} \ln \mu_{F}^{2}}=\gamma_{N}\left(\alpha_{S}\left(\mu_{F}^{2}\right)\right) E_{N}\left(\mu_{F}^{2}, \mu_{0 F}^{2}\right)
$$

with the boundary condition $E_{N}\left(\mu_{0 F}^{2}, \mu_{0 F}^{2}\right)=1$. In this equation, the anomalous dimension $\gamma$ is related to the well-known time-like Altarelli-Parisi splitting functions in Mellin space $P_{N}$ [52-57], (up to the second order in $\alpha_{s}$ ) by

$$
\gamma_{N}\left(\alpha_{S}\right)=\frac{\alpha_{S}}{2 \pi} P_{N}^{(0)}+\left(\frac{\alpha_{S}}{2 \pi}\right)^{2} P_{N}^{(1)}+\mathcal{O}\left(\alpha_{S}^{3}\right)
$$

For heavy quark fragmentation, the starting point of the perturbative evolution $\mu_{0 F}$ is chosen to be of the same order as $m$, the mass of the heavy quark. As such it is a perturbative scale (i.e. $m \gg \Lambda_{\mathrm{QCD}}$ ). As a consequence, unlike the parton-to-hadron fragmentation function defined at the initial scale $\mu_{0 F, H}$ close to $\Lambda_{\mathrm{QCD}}$, the initial condition $D_{N}^{\text {ini }}$ for the parton-to-(heavy quark) fragmentation function which depends on this perturbative starting scale $\mu_{0 F}$, can be computed in perturbation theory as a power series in $\alpha_{s}$. At leading order, we have $D^{\text {ini }}(x)=\delta(1-x)$ (and therefore $D_{N}^{\text {ini }}=1$ ), which expresses nothing but the fact that at leading order, a $b$-flavoured jet is a $b$ quark. As such the LO initial condition is trivial and independent of the mass $m$ of the heavy-quark.

In equation (78), the process dependence of the inclusive cross section is entirely contained in the coefficient function $C_{N}^{\left(e^{+} e^{-}\right)}$. It denotes the Mellin transform of the $e^{+} e^{-}$coefficient function in the $\overline{M S}$ scheme. It is given in [58]. For conciseness it is not presented here, its behaviour for large Mellin moment $N$ is given by,

$$
\begin{aligned}
C_{N}^{\left(e^{+} e^{-}\right)}=1+\frac{\alpha_{S}\left(\mu^{2}\right)}{\pi} C_{F}\left(-\left(\ln N+\gamma_{E}\right.\right. & \left.-\frac{3}{4}\right) \ln \left(\frac{E_{\mathrm{cm}}^{2}}{\mu_{F}^{2}}\right)+\frac{1}{2} \ln ^{2} N+\left(\frac{3}{4}+\gamma_{E}\right) \ln N \\
& \left.+\left(\frac{5}{12} \pi^{2}-\frac{9}{4}+\frac{1}{2} \gamma_{E}^{2}+\frac{3}{4} \gamma_{E}\right)+\mathcal{O}\left(\frac{1}{N}\right)\right) .
\end{aligned}
$$

The perturbative fragmentation function $D_{N}$, as well as its components the evolution operator $E_{N}$ and the initial fragmentation function $D_{\text {ini }}$ are instead universal. Beyond leading order, those depend 
on the factorization scales $\mu_{F}$ and $\mu_{0 F}$ as well as on the renormalization scales $\mu$ and $\mu_{0}$. We start by describing the required evolution operator $E_{N}$ needed for our comparison.

The resummation of large collinear logarithms is performed by solving the AP equations perturbatively and by setting factorization and renormalization scales equal. This is realized by setting $\mu_{F} \approx \mu \approx E_{\mathrm{cm}}$ and $\mu_{0} \approx \mu_{0 F} \approx m$.

Using in addition the second-order expansion of the anomalous dimensions as given in equation (81) the evolution operator for the non-singlet channel reads,

$$
E_{N}\left(\mu_{F}^{2}, \mu_{0 F}^{2}\right)=\left(\frac{\alpha_{S}\left(\mu_{0 F}^{2}\right)}{\alpha_{S}\left(\mu_{F}^{2}\right)}\right)^{\frac{P_{N}^{(0)}}{2 \pi b_{0}}} \exp \left(\frac{\alpha_{S}\left(\mu_{0 F}^{2}\right)-\alpha_{S}\left(\mu_{F}^{2}\right)}{4 \pi^{2} b_{0}}\left(P_{N}^{(1)}-\frac{2 \pi b_{1}}{b_{0}} P_{N}^{(0)}\right)\right) .
$$

Using this equation, one sees that the leading collinear logarithms of the form $\left(\alpha_{s} \ln \left(E_{\mathrm{cm}}^{2} / m^{2}\right)\right)^{n}$ of equation (78) are resummed by combining the LO expression of the evolution operator with the LO expressions of the initial condition $D_{\text {ini }}$ and the LO expression of the coefficient function $C^{e^{+} e^{-}}$. Since at this order, these three ingredients do not depend on the heavy quark mass $m$, it corresponds to using a massless calculation to describe the heavy quark fragmentation process, which is a very crude approximation. The resummation of the next-to-leading collinear terms of the form $\alpha_{s}\left(\alpha_{s} \ln \left(E_{\mathrm{cm}}^{2} / m^{2}\right)\right)^{n}$ in the evolution operator given in equation (83) above requires the equivalent NLO expressions for these three components which are now mass-dependent. As a consequence, although the collinear-enhanced logarithms are formally resummed in VINCIA only at the LL level, to compare our predictions, we will use the full evolution operator $E_{N}$ defined above in equation (83) which includes subleading logarithmic effects. The comparison can in fact only be made with the analytic result which shows a clear mass-dependent behaviour.

To come to $D_{N}^{\mathrm{ini}}$, as mentioned before, the initial scale $\mu_{0 F}$ is perturbative as it is chosen of the order of the heavy-quark mass $m$ and by this choice, the appearance of large logarithms of the ratio $\mu_{0 F} / m$ are avoided in the perturbative initial condition $D_{N}^{\text {ini }}$. The perturbative initial condition up to order $\alpha_{S}$ which only resums the collinear-enhanced logarithms of the form $E_{\mathrm{cm}}^{2} / m^{2}$ is given below as,

$$
\begin{aligned}
& D_{N}^{\mathrm{ini}}\left(\alpha_{S}\left(\mu_{0}^{2}\right), \mu_{0}^{2}, \mu_{0 F}^{2}, m^{2}\right)= \\
& \qquad 1+\frac{\alpha_{S}\left(\mu_{0}^{2}\right)}{2 \pi} C_{F} \int_{0}^{1} \mathrm{~d} z\left(z^{N-1}-1\right)\left(\frac{1+z^{2}}{1-z}\left(\ln \frac{\mu_{0 F}^{2}}{(1-z)^{2} m^{2}}-1\right)\right)+\mathcal{O}\left(\alpha_{S}^{2}\right) .
\end{aligned}
$$

The corresponding expression in the large- $N$ limit is given by,

$$
\begin{aligned}
& D_{N}^{\mathrm{ini}}\left(\alpha_{S}\left(\mu_{0}^{2}\right), \mu_{0}^{2}, \mu_{0 F}^{2}, m^{2}\right)= \\
& \quad 1+\frac{\alpha_{S}\left(\mu_{0}^{2}\right)}{\pi} C_{F}\left(-\ln ^{2} N+\left(\ln \frac{m^{2}}{\mu_{0 F}^{2}}-2 \gamma_{E}+1\right) \ln N+\mathcal{O}(1)\right)+\mathcal{O}\left(\alpha_{S}^{2}\right) .
\end{aligned}
$$

As can be seen from equation (82) for $C_{N}$ and equation (85) for $D_{N}^{\text {ini }}$ given in the large $N$ limit, for $1-x \ll 1$ (corresponding to $N \gg 1$ ), both the perturbative initial condition and the $e^{+} e^{-}$coefficient function cannot be computed in fixed-order perturbation theory as they contain logarithmic contributions proportional to $\ln N$ and $\ln ^{2} N$ respectively. Those logarithmic contributions, arising through the radiation of soft gluons from the heavy quarks at the renormalization scale $\mu_{0} \approx m$, spoil the convergence 
of the fixed order perturbative expansion at large $N$ (or equivalently at large $x$ ) and have therefore to be resummed.

Note that we expect the soft-gluon effect to be quantitatively more important for the initial condition of the heavy-quark fragmentation function $D_{\text {ini }}$ than for the coefficient function $C^{e^{+} e^{-}}$since in the first case this soft-gluon or so-called Sudakov effect is controlled by the coupling $\alpha_{s}\left(\mu_{0}^{2}\right)$ which is larger than $\alpha_{s}\left(\mu_{F}^{2}\right)$ present in the partonic cross section $C^{e^{+} e^{-}}$instead.

In [33], this soft-gluon resummation was performed to NLL accuracy for both the coefficient function and the perturbative initial condition. The expression of the coefficient function including soft-gluon resummation effects given in Mellin space, which we shall call soft-gluon-resummed part of the coefficient function is denoted by $C_{N}^{S}$ reads,

$$
C_{N}^{\mathrm{S}}\left(\alpha_{S}\left(\mu^{2}\right), E_{\mathrm{cm}}^{2}, \mu^{2}, \mu_{F}^{2}\right)=\exp \left(\ln N g^{(1)}(\lambda)+g^{(2)}\left(\lambda, \frac{E_{\mathrm{cm}}^{2}}{\mu^{2}}, \frac{E_{\mathrm{cm}}^{2}}{\mu_{F}^{2}}\right)\right)
$$

with $\lambda=b_{0} \alpha_{S}\left(\mu^{2}\right) \ln N$ and

$$
\begin{aligned}
g^{(1)}(\lambda)= & \frac{A^{(1)}}{\pi b_{0} \lambda}(\lambda+(1-\lambda) \ln (1-\lambda)), \\
g^{(2)}\left(\lambda, \frac{E_{\mathrm{cm}}^{2}}{\mu^{2}}, \frac{E_{\mathrm{cm}}^{2}}{\mu_{F}^{2}}\right)= & \frac{A^{(1)} b_{1}}{2 \pi b_{0}^{3}}\left(2 \lambda+2 \ln (1-\lambda)+\ln ^{2}(1-\lambda)\right)+\frac{B^{(1)}-2 A^{(1)} \gamma_{E}}{2 \pi b_{0}} \ln (1-\lambda) \\
& -\frac{1}{\pi b_{0}}(\lambda+\ln (1-\lambda))\left(\frac{A^{(2)}}{\pi b_{0}}-A^{(1)} \ln \frac{E_{\mathrm{cm}}^{2}}{\mu^{2}}\right)-\frac{A^{(1)}}{\pi b_{0}} \lambda \ln \frac{E_{\mathrm{cm}}^{2}}{\mu_{F}^{2}} .
\end{aligned}
$$

The coefficients $A^{(1)}, A^{(2)}$ and $B^{(1)}$ are given in the $\overline{M S}$ scheme by

$$
\begin{aligned}
& A^{(1)}=C_{F}, A^{(2)}=\frac{1}{2} C_{F}\left(C_{A}\left(\frac{67}{18}-\frac{\pi^{2}}{6}-\frac{5}{9} N_{f}\right)\right), \\
& B^{(1)}=-\frac{3}{2} C_{F} .
\end{aligned}
$$

The initial condition $D_{\text {ini }}$ including soft-gluon resummation which we shall call soft-gluon-resummed part of the initial condition is denoted by $D_{N}^{\text {ini,S }}$ and reads,

$$
D_{N}^{\text {ini, }}\left(\alpha_{S}\left(\mu_{0}^{2}\right), \mu_{0}^{2}, \mu_{0 F}^{2}, m^{2}\right)=\exp \left(\ln N g_{\text {ini }}^{(1)}\left(\lambda_{0}\right)+g_{\text {ini }}^{(2)}\left(\lambda_{0}, \frac{m^{2}}{\mu_{0}^{2}}, \frac{m^{2}}{\mu_{0 F}^{2}}\right)\right)
$$


with $\lambda_{0}=b_{0} \alpha_{S}\left(\mu_{0}^{2}\right) \ln N$ and

$$
\begin{aligned}
g_{\mathrm{ini}}^{(1)}\left(\lambda_{0}\right)= & -\frac{A^{(1)}}{2 \pi b_{0} \lambda_{0}}\left(2 \lambda_{0}+\left(1-2 \lambda_{0}\right) \ln \left(1-2 \lambda_{0}\right)\right), \\
g_{\mathrm{ini}}^{(2)}\left(\lambda_{0}, \frac{m^{2}}{\mu_{0}^{2}}, \frac{m^{2}}{\mu_{0 F}^{2}}\right)= & \frac{A^{(1)}}{2 \pi b_{0}}\left(\ln \frac{\mu_{0 F}^{2}}{m^{2}}+2 \gamma_{E}\right) \ln \left(1-2 \lambda_{0}\right) \\
& -\frac{A^{(1) b_{1}}}{4 \pi b_{0}^{3}}\left(4 \lambda_{0}+2 \ln \left(1-2 \lambda_{0}\right)+\ln ^{2}\left(1-2 \lambda_{0}\right)\right) \\
& +\frac{1}{2 \pi b_{0}}\left(2 \lambda_{0}+\ln \left(1-2 \lambda_{0}\right)\right)\left(\frac{A^{(2)}}{\pi b_{0}}+A^{(1)} \ln \frac{\mu_{0}^{2}}{\mu_{0 F}^{2}}\right) \\
& +\frac{H^{(1)}}{2 \pi b_{0}} \ln \left(1-2 \lambda_{0}\right) .
\end{aligned}
$$

From the formulae above, one can see that the soft-gluon resummed parts of the coefficient function and of the initial condition are not valid for arbitrarily large $N$ values. Those have branch cuts in the complex $N$-plane, the former starting at $N=\exp \left(1 /\left(b_{0} \alpha_{S}\left(\mu^{2}\right)\right)\right)$, the latter at $N=\exp \left(1 /\left(2 b_{0} \alpha_{S}\left(\mu_{0}^{2}\right)\right)\right)$. This behaviour signals the onset of non-perturbative physics at values of $x$ very close to 1 . Keeping this fact in mind, we will restrict our comparisons of the VINCIA results with the soft-gluon resummed calculation to moderate $(N \leq 25)$ Mellin moments.

The final step is to match the soft-gluon resummed part of the coefficient function, equations (86), and initial condition, equations (89), to the fixed-order result in such a way that the truncation of the matched result reproduces the fixed-order result and the logarithmic accuracy of the resummed part is preserved. This requirement does not fix the matched result uniquely, however. In [33], the matching was performed additively. ${ }^{10}$

The final NLO +NLL resummed expression for the N moments of the (non-singlet) initial condition denoted by $D_{N}^{\text {ini, matched }}$ is given by,

$$
D_{N}^{\mathrm{ini}, \text { matched }}=D_{N}^{\mathrm{ini}}+\left(D_{N}^{\mathrm{ini}, \mathrm{S}}-\left.D_{N}^{\mathrm{ini}, \mathrm{S}}\right|_{\alpha_{S}}\right)
$$

In this equation, $D_{N}^{\mathrm{ini}}$ is given above in equation (5.1), $D_{N}^{\mathrm{ini}, \mathrm{S}}$ is the Sudakov-resummed part given in equation (89) while $\left.D_{N}^{\text {ini, S }}\right|_{\alpha_{S}}$ denotes the expansion of this expression $D_{N}^{\text {ini, S }}$ given in equation (89) to the first order in $\alpha_{S}$. The definition of the matched $e^{+} e^{-}$coefficient function is analogous to equation (91), i.e. we have,

$$
C_{N}^{\text {matched }}=C_{N}+\left(C_{N}^{\mathrm{S}}-\left.C_{N}^{\mathrm{S}}\right|_{\alpha_{S}}\right)
$$

In this equation, $C_{N}$ is the full $e^{+} e^{-}$coefficient function at $\mathcal{O}\left(\alpha_{s}\right)$ given in [58], $C_{N}^{\mathrm{S}}$ is the resummed part as given in equation (86) above and $\left.C_{N}^{\mathrm{S}}\right|_{\alpha_{S}}$ corresponds to its expansion at order $\alpha_{s}$.

\footnotetext{
${ }^{10}$ The numerical difference of this matching scheme to the so-called "log-R matching scheme" used for example in [59] for the same observable is small for the parameter values we consider.
} 


\subsection{Comparison with VINCIA}

We now compare the analytical calculation of the fragmentation function $\mathcal{D}\left(E_{\mathrm{cm}}^{2}, m^{2}\right)$, equation (78), described in the previous subsection, to VINCIA results transformed to Mellin space. For the analytical calculation, we use the evolution operator defined by equation (83) and consider two possibilities for the

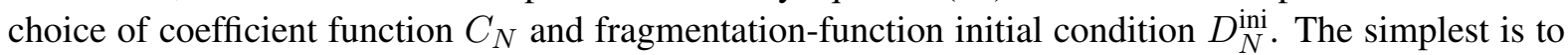
take the analytic results obtained using the fixed-order coefficient function given in [58], and the form for $D_{N}^{\text {ini }}$ which only resums the collinear-enhanced logarithms given in equation (78). Alternatively, we use the equivalent matched expressions defined above in equations (91) and equation (92), which include soft-gluon resummation effects up to the NLL accuracy.

In order to do the comparison appropriately, we need to fix a kinematical range where both the analytical results and the prediction from VINCIA are reliable. The range of validity of the analytic resummation calculation was discussed in the previous subsection. We expect it to work for a centre-ofmass energy $E_{\mathrm{cm}}$ such that $E_{\mathrm{cm}} \gg m$ and for $m \gg \Lambda_{\mathrm{QCD}}$. For VINCIA, the requirements for the mass of the heavy quark $m$ are given by,

$$
Q_{\text {stop }} \ll m_{Q} \ll E_{\text {cm }},
$$

where $Q_{\text {stop }}$ denotes the emission scale at which VINCIA would make the transition to a hadronization model. The first hierarchy $Q_{\text {stop }} \ll m_{Q}$ is necessary to ensure that gluons which are soft compared to the quark mass can be emitted, whereas the second hierarchy $m_{Q} \ll E_{\mathrm{cm}}$ ensures that the quasi-collinear logarithms play an important role.

Furthermore, since the accuracy of VINCIA is formally LL, there still remain considerable uncertainties. The uncertainty due to the choice of finite terms, estimated by using the "MIN" and "MAX" antenna sets defined in section 2.5, was found to be small in comparison to the uncertainty coming from the choice of renormalization scale. Hence we only consider the default antennae set for our results here. The renormalization-scale choice was then varied between $\mu_{R}=2 p_{\perp}$ and $\mu_{R}=p_{\perp} / 2$, to define the uncertainty range. (Note that, in the parton shower context, we do not have an explicit factorization scale, the evolution variable $Q_{E}$ has the role of a factorization scale instead.)

The predictions obtained with the analytic resummation calculation will be taken at factorization and renormalization scale respectively given by $\mu_{F}=\mu=Q$ and $\mu_{0}=\mu_{0 F}=m$.

The $x$-space fragmentation function obtained with VINCIA for a centre-of-mass energy of $10^{4} \mathrm{GeV}$, quark mass $m_{Q} / E_{\mathrm{cm}}=0.02$ and $\alpha_{S}\left(m_{Z}^{2}\right)=0.139$ (using one-loop runing) is shown in the left-hand pane of figure 16, in the range $0.8 \leq x \leq 1$. The right-hand pane shows the comparison to the analytical results in Mellin space, in the range $1<N<25$, using the same parameters as for the VINCIA result. We find reasonable agreement between the two predictions over this range. The VINCIA prediction reproduces the main feature of the analytic resummation calculation. Due to its large uncertainty, it cannot however distinguish clearly between the different analytic predictions. ${ }^{11}$ We find comparable agreement for light $\left(m_{Q} \lesssim 0.04 E_{\mathrm{cm}}\right)$ quarks, under the condition that the dependence of the VINCIA result on the cutoff $Q_{\text {stop }}$ is small.

\footnotetext{
${ }^{11}$ There are parameter values for which the resummation of soft-gluon logarithms has a much more pronounced effect than in figure 16. It turned out, however, that the dependence of the VINCIA results on the cutoff $Q_{\text {stop }}$ is too large to allow for a comparison in these cases.
} 

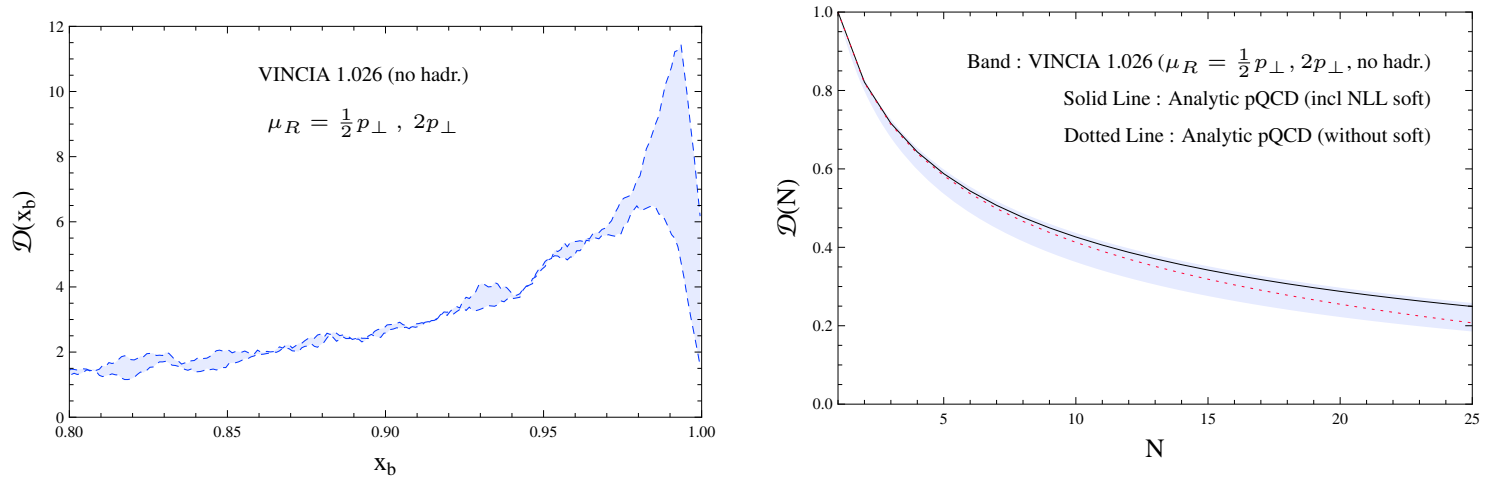

Figure 16: Left: the $\mathcal{D}\left(x_{b}\right)$ distribution obtained with VINCIA with hadronization switched off, i.e., at $Q_{\text {stop }}=$ $2 p_{\perp \text { stop }}=1 \mathrm{GeV}$. The shaded band shows the uncertainty obtained by varying the renormalization scale in $\alpha_{S}$ by a factor of 2 and $1 / 2$ respectively (with scale-stabilization switched off, see [6]). Right: the Mellin transform, $\mathcal{D}(N)$, including comparisons to analytic resummation with (solid) and without (dotted) soft-gluon resummation at NLL. $E_{\mathrm{cm}}=10^{4} \mathrm{GeV}, m_{Q}=0.02 E_{\mathrm{cm}}, \alpha_{S}\left(m_{Z}^{2}\right)=0.139$. The VINCIA predictions use the default dipoleantenna set, strong ordering of the emissions in $p_{\perp}$, no matching, and no secondary quark-antiquark production.

\section{Comparison to b-tagged experimental data}

As a final cross-check, we include three basic comparisons to data published by the SLD [60], DELPHI [61], and L3 [62] experiments. We do not intend this to represent a full-fledged phenomenological study of $b$ fragmentation in VINCIA. Rather, we wish to demonstrate that our implementation of mass effects in VINCIA yields sensible numbers also when compared directly to experimental data, including the effects of hadronization.

We also include comparisons to default PYTHIA 8.150, on the same distributions. (This is trivial for us to do - we merely switch VINCIA off.) The resulting comparison represents a further validation and cross check (of both models) since the shower formalisms are quite different between PYTHIA and VINCIA, especially for massive particles. We also obtain a concrete check that the events generated by VINCIA are being treated by PYTHIA's string model of hadronization in a consistent manner.

For PYTHIA, we use the default parameters of PYTHIA 8.150 [63]. We also note that default PYTHIA includes matching through $Z \rightarrow 3$ partons. For VINCIA, we use the VINCIA-specific tune of the light-flavour parameters reported in [6] and include matching through $Z \rightarrow 5$ partons. Since tuning is not our main purpose here, we have not attempted to retune PYTHIA's $b$-specific non-perturbative parameters to (re)optimize them for use with VINCIA. It is therefore possible that some further improvements could be made in the non-perturbative description.

In figure 17, we show the fragmentation function (a) for $b$ quarks at the parton level ${ }^{12}$ and (b) at the hadron level, compared to hadron-level SLD data for weakly decaying $B$ mesons [60]. The top panes show the distributions normalized to unity, and the bottom panes show the ratios of theory to data. In the bottom panes, the inner (lighter) yellow bands indicate the statistical uncertainty, and the outer

\footnotetext{
${ }^{12}$ Obtained by switching off PYTHIA's hadronization model, i.e., the $b$ quarks are evolved perturbatively to a scale $Q_{\text {stop }}=$ $2 p_{\perp \text { had }}=1 \mathrm{GeV}$ (or $2 p_{\perp \text { evol }}=0.8 \mathrm{GeV}$ in standalone PYTHIA). We ignore the slight difference in the exact value and definition of $p_{\perp}$ between PYTHIA and VINCIA, see [64] for a discussion.
} 


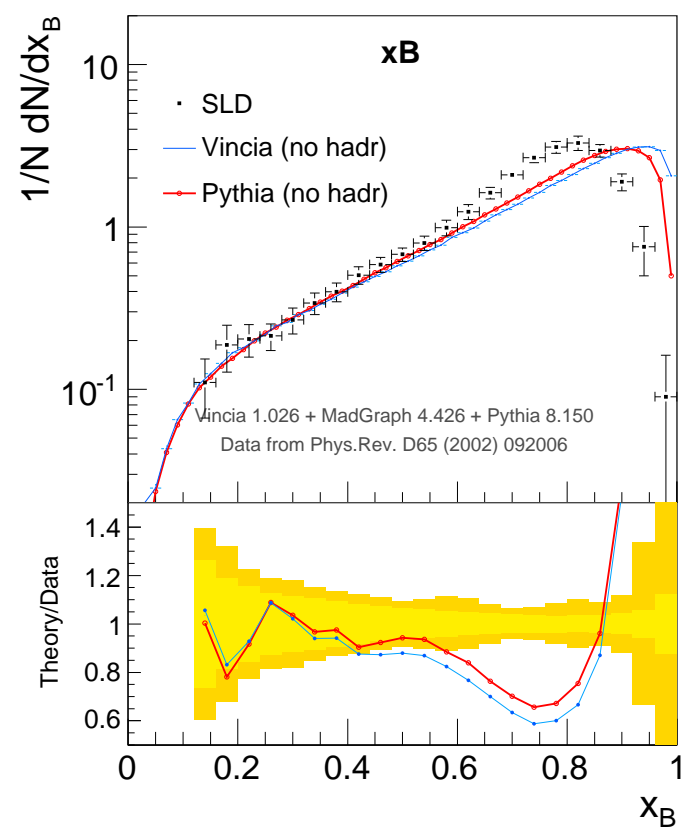

(a) Hadronization OFF

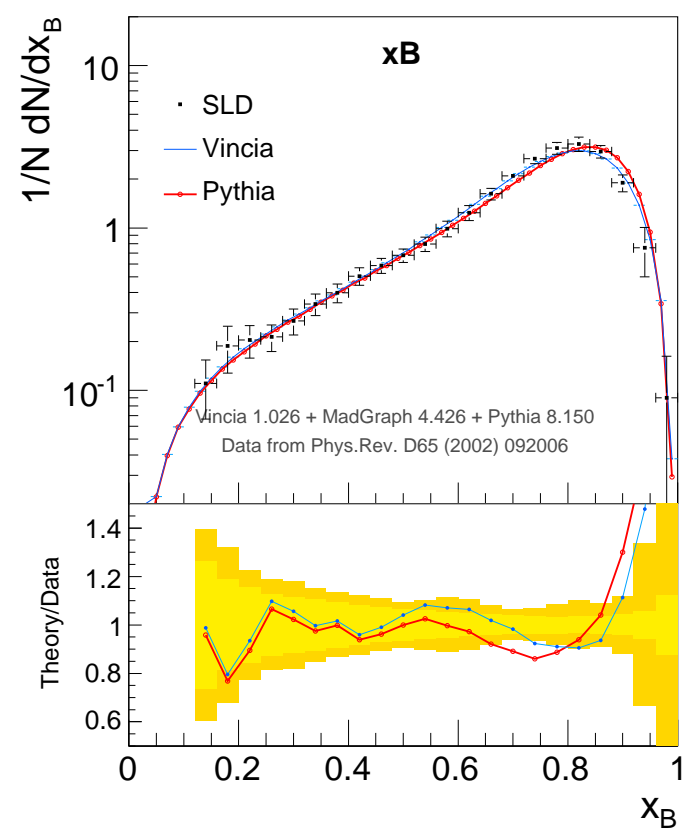

(b) Hadronization $\mathrm{ON}$

Figure 17: VINCIA (thin lines) and PYTHIA 8 (thick lines), before (left) and after (right) hadronization, compared to SLD [60] data on the fragmentation function for weakly decaying $B$ mesons

(darker) bands indicate the combined statistical plus systematical uncertainty, added linearly. Note that the vertical error bars on the Monte Carlo predictions correspond to $\pm 1.645 \sigma$ statistical uncertainty from the number of generated MC points, equivalent to $90 \%$ confidence. This is the default for how statistical MC uncertainties are displayed by VINCIA's plotting tool.

Comparing the two panes of figure 17, we conclude that the non-perturbative corrections are significant in the region above $x_{B} \sim 0.5$, while the spectrum at lower $x_{B}$ is dominated by the perturbative prediction, for which the two codes are in good agreement, both with each other and with the data. In the high- $x_{B}$ region, VINCIA generates a slightly harder parton-level spectrum (peaked at higher $x_{B}$ ) than PYTHIA. That is, VINCIA generates slightly less perturbative radiation. After hadronization, the VINCIA spectrum is somewhat softer than the PYTHIA one. The total non-perturbative component of the "parton-to-hadron" correction would therefore be evaluated as being slightly larger for VINCIA than for PYTHIA. The main properties of this correction are driven by the tuning to light flavours. As mentioned above, a dedicated $b$-specific tuning has not yet been carried out. Nonetheless, we note that the fragmentation spectrum obtained with the default tuning already appears to be in reasonable agreement with the data, cf. the right-hand pane of figure 17.

In figure 18, we have used FASTJET [65] to compare VINCIA and PYTHIA to DELPHI data [61] on $R_{3}^{b l}$, the ratio of the $y_{23}$ distribution in $b$-tagged events to light-flavour events, with $y_{23}$ the dimensionless resolution scale at which the event goes from being a 2- to a 3-jet event, according to the $k_{T}$ clustering algorithm (using the $E$ scheme). This distribution is sensitive to the value of the $b$-quark 


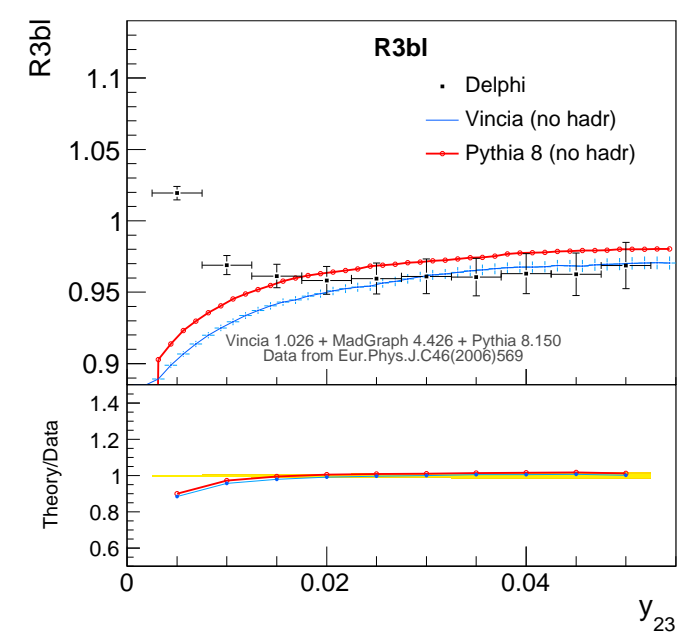

(a) Hadronization OFF

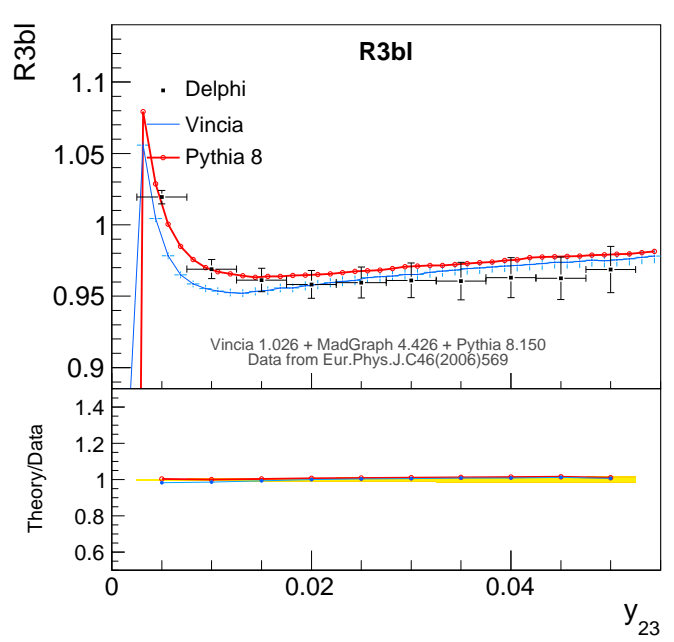

(b) Hadronization ON

Figure 18: VINCIA (thin lines) and PYTHIA 8 (thick lines), before (left) and after (right) hadronization, compared to DELPHI [61] data on the ratio of $y_{23}$ in $b$ - vs. light-flavour events.

mass. Comparing left- (without hadronization) to right-hand (with hadronization) panes, we see that hadronization effects are large in the region below, roughly, $y_{23} \sim 0.02$. Above that value, the deviation from unity is generated mainly by perturbative quark mass effects. Both models use the default PYTHIA value of $m_{b}=4.8 \mathrm{GeV}$, which appears to give a reasonable agreement with the measurement.

In figure 19, we show a similar comparison for the Thrust event shape variable in $b$-tagged events, compared to a measurement performed by the L3 collaboration [62]. Comparing (a) parton- to (b) hadron-level results, an interesting pattern can be seen, for both PYTHIA and VINCIA. The nonperturbative corrections are significant not only at low values of $\tau=1-T<0.1$, but also around $\tau=1 / 3$, shown with a vertical dotted line in the upper panes of the plots. At this point, the distribution changes slope ${ }^{13}$; thus, even though hadronization effects are parametrically strongly suppressed in that region, the small "smearing" they provide of the underlying perturbative prediction becomes relatively more important at exactly that point. With hadronization effects included, both models describe the data acceptably well. Combined with the good agreement found with the light-flavour variables included in [6], we conclude that the VINCIA code can at this point be considered validated as a Monte Carlo model for final-state showering and hadronization effects.

\section{Conclusions and Outlook}

A precise description of processes involving heavy coloured particles is of prime importance to the physics programme at current and future high-energy collider facilities. In this article, we have extended the timelike dipole-antenna shower formalism of $[5,6]$ to include massive fermions. Advantages of this treatment include the exact on-shell phase-space factorization which is inherent to the antenna formalism

\footnotetext{
${ }^{13}$ Due to the underlying change from a 3-parton to a 4-parton quantity that the Thrust variable undergoes at that point.
} 


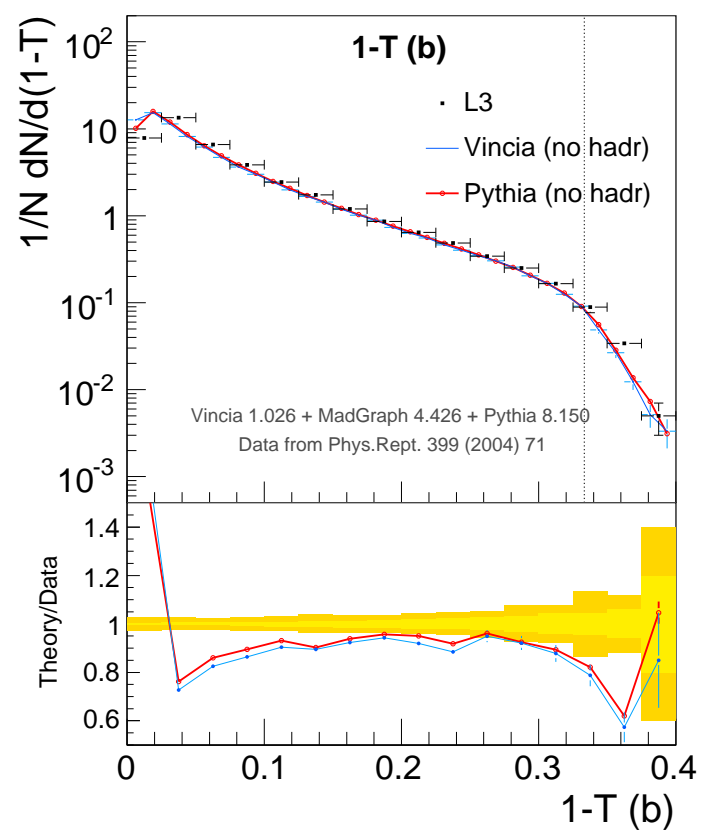

(a) Hadronization OFF

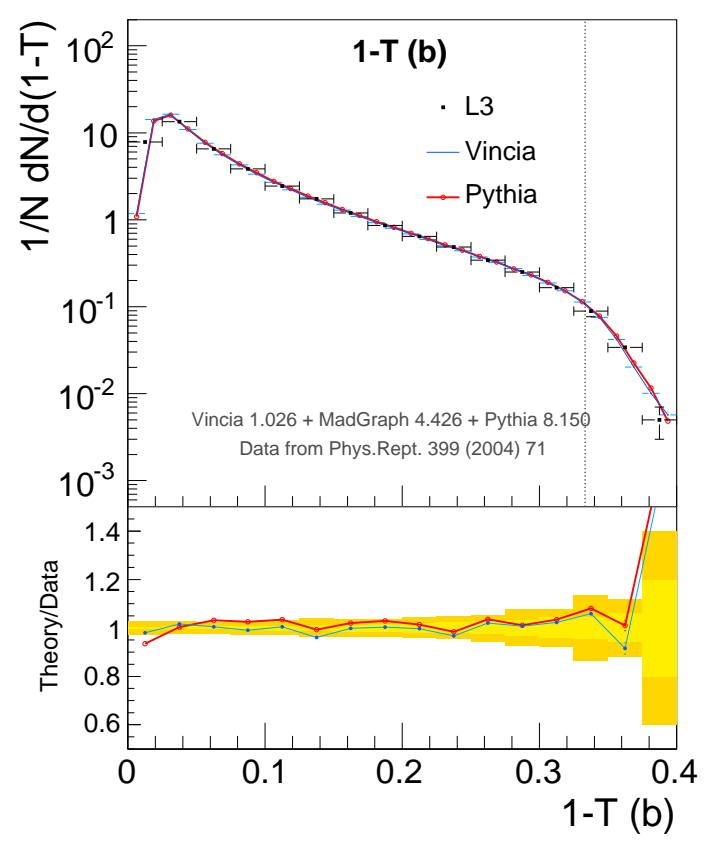

(b) Hadronization ON

Figure 19: VINCIA (thin lines) and PYTHIA 8 (thick lines), before (left) and after (right) hadronization, compared to L3 [62] data on Thrust in $b$-tagged events.

[12-14], the ability to vary the remaining ambiguous parts of the calculation in a similar manner as was already done for the massless case but now including explicit mass-dependent terms, and the smooth merging with the "GKS" matching formalism [6].

In this paper, we have shown evidence, through extensive comparisons to other calculational methods, that the algorithm is physically sensible and that it can be expected to yield reasonably precise results over large parts of the phase space. Furthermore, using the GKS formalism, it can be matched to leading-order matrix elements over all of phase space up to any given fixed order (in practice, the limit is currently Born +4 partons), which should help to give a systematic improvement even for fairly soft and/or collinear emissions.

Although we have not attempted a dedicated tuning of the non-perturbative fragmentation parameters for heavy quarks, the preliminary tuning of the massless parameters reported in [6], combined with the PYTHIA 8 defaults for the $b$-specific ones, appears to give a reasonably good description of the $B$ fragmentation function and of $b$-tagged event shapes and jet rates at the $Z$ pole.

For a full-fledged application to physics at hadron colliders, two further ingredients remain to be developed: an extension of the formalism to spacelike (initial-state) showers, and the inclusion of finitewidth decays, which can be important in the description of (chains of) resonance decays. Nonetheless, the step taken here is a necessary prerequisite, and can already be used to study questions involving final-state $c$ and $b$ quark fragmentation. 


\section{Acknowledgments}

We thank W. Giele and D. Kosower for many useful discussions and comments on the manuscript. This work was carried out partly at, and with the support of, the Institute of Theoretical Physics at ETH Zurich and the CERN Theory Division. The authors acknowledge both of these institutions for their kind hospitality.

This work was supported in part by the Marie Curie research training network "MCnet" (contract number MRTN-CT-2006-035606), by the Swiss National Science Foundation (SNF) under contract PP0022-118864 and by the European Commission through the 'LHCPhenoNet' Initial Training Network PITN-GA-2010-264564', which are hereby acknowledged.

\section{References}

[1] P. Z. Skands and D. Wicke, Eur.Phys.J. C52, 133 (2007), hep-ph/0703081.

[2] S. Fleming, A. H. Hoang, S. Mantry, and I. W. Stewart, Phys.Rev. D77, 074010 (2008), hep$\mathrm{ph} / 0703207$.

[3] A. Buckley et al., Phys.Rept. 504, 145 (2011), 1101.2599.

[4] E. Norrbin and T. Sjöstrand, Nucl.Phys. B603, 297 (2001), hep-ph/0010012.

[5] W. T. Giele, D. A. Kosower, and P. Z. Skands, Phys.Rev. D78, 014026 (2008), 0707.3652.

[6] W. Giele, D. Kosower, and P. Skands, Phys.Rev. D84, 054003 (2011), 1102.2126.

[7] A. J. Larkoski and M. E. Peskin, Phys.Rev. D84, 034034 (2011), 1106.2182.

[8] L. Lönnblad, Comput. Phys. Commun. 71, 15 (1992).

[9] J.-C. Winter and F. Krauss, JHEP 0807, 040 (2008), 0712.3913.

[10] A. Gehrmann-De Ridder, T. Gehrmann, and E. W. N. Glover, Phys. Lett. B612, 36 (2005), hep$\mathrm{ph} / 0501291$.

[11] A. Gehrmann-De Ridder, T. Gehrmann, and E. Glover, Phys.Lett. B612, 49 (2005), hep$\mathrm{ph} / 0502110$.

[12] A. Gehrmann-De Ridder, T. Gehrmann, and E. Glover, JHEP 0509, 056 (2005), hep-ph/0505111, Erratum added online, 8/18/06.

[13] A. Gehrmann-De Ridder and M. Ritzmann, JHEP 0907, 041 (2009), 0904.3297.

[14] G. Abelof and A. Gehrmann-De Ridder, JHEP 1104, 063 (2011), 1102.2443.

[15] K. Hamilton and P. Nason, JHEP 1006, 039 (2010), 1004.1764.

[16] S. Catani, F. Krauss, R. Kuhn, and B. R. Webber, JHEP 11, 063 (2001), hep-ph/0109231.

[17] F. Krauss, JHEP 08, 015 (2002), hep-ph/0205283. 
[18] L. Lönnblad, JHEP 05, 046 (2002), hep-ph/0112284.

[19] S. Mrenna and P. Richardson, JHEP 05, 040 (2004), hep-ph/0312274.

[20] N. Lavesson and L. Lönnblad, JHEP 04, 085 (2008), 0712.2966.

[21] J. Alwall et al., Eur. Phys. J. C53, 473 (2008), 0706.2569.

[22] J. Lopez-Villarejo and P. Skands, (2011), 1109.3608.

[23] D0 Collaboration, V. M. Abazov et al., Phys.Lett. B703, 422 (2011), 1104.2887.

[24] A. H. Hoang and I. W. Stewart, Nucl.Phys.Proc.Suppl. 185, 220 (2008), 0808.0222.

[25] T. Sjöstrand, S. Mrenna, and P. Skands, Comput. Phys. Commun. 178, 852 (2008), 0710.3820.

[26] VINCIA homepage, http://projects.hepforge.org/vincia.

[27] G. Gustafson and U. Pettersson, Nucl. Phys. B306, 746 (1988).

[28] D. A. Kosower, Phys. Rev. D57, 5410 (1998), hep-ph/9710213.

[29] D. A. Kosower, Phys.Rev. D71, 045016 (2005), hep-ph/0311272.

[30] S. Catani, S. Dittmaier, M. H. Seymour, and Z. Trocsanyi, Nucl. Phys. B627, 189 (2002), hep$\mathrm{ph} / 0201036$.

[31] S. Catani, S. Dittmaier, and Z. Trocsanyi, Phys. Lett. B500, 149 (2001), hep-ph/0011222.

[32] S. Frixione, Z. Kunszt, and A. Signer, Nucl.Phys. B467, 399 (1996), hep-ph/9512328.

[33] M. Cacciari and S. Catani, Nucl.Phys. B617, 253 (2001), hep-ph/0107138.

[34] G. Altarelli and G. Parisi, Nucl.Phys. B126, 298 (1977).

[35] S. Catani and M. Seymour, Phys.Lett. B378, 287 (1996), hep-ph/9602277.

[36] P. Skands, (2011), 1104.2863.

[37] P. Z. Skands and S. Weinzierl, Phys.Rev. D79, 074021 (2009), 0903.2150.

[38] B. Andersson, G. Gustafson, and C. Sjögren, Nucl.Phys. B380, 391 (1992).

[39] F. James, Rept.Prog.Phys. 43, 1145 (1980).

[40] S. Weinzierl, (2000), hep-ph/0006269, Topical lectures given at the Research School Subatomic physics, Amsterdam, the Netherlands, June 2000.

[41] M. Seymour, Z.Phys. C63, 99 (1994), Revised Version.

[42] M. Bengtsson and T. Sjöstrand, Phys.Lett. B185, 435 (1987).

[43] C. W. Bauer, F. J. Tackmann, and J. Thaler, JHEP 0812, 011 (2008), 0801.4028. 
[44] J. Alwall et al., JHEP 0709, 028 (2007), 0706.2334.

[45] R. Kleiss, W. Stirling, and S. Ellis, Comput.Phys.Commun. 40, 359 (1986).

[46] B. Mele and P. Nason, Nucl.Phys. B361, 626 (1991).

[47] M. Cacciari and M. Greco, Nucl.Phys. B421, 530 (1994), hep-ph/9311260.

[48] C. Peterson, D. Schlatter, I. Schmitt, and P. M. Zerwas, Phys.Rev. D27, 105 (1983).

[49] J. Owens, Phys.Lett. B76, 85 (1978).

[50] S. Catani, M. L. Mangano, P. Nason, and L. Trentadue, Phys.Lett. B378, 329 (1996), hep$\mathrm{ph} / 9602208$.

[51] S. Catani, M. L. Mangano, P. Nason, and L. Trentadue, Nucl.Phys. B478, 273 (1996), hep$\mathrm{ph} / 9604351$.

[52] G. Curci, W. Furmanski, and R. Petronzio, Nucl.Phys. B175, 27 (1980).

[53] W. Furmanski and R. Petronzio, Z.Phys. C11, 293 (1982).

[54] J. Kalinowski, K. Konishi, P. Scharbach, and T. Taylor, Nucl.Phys. B181, 253 (1981).

[55] E. Floratos, R. Lacaze, and C. Kounnas, Phys.Lett. B98, 89 (1981).

[56] I. Antoniadis and E. Floratos, Nucl.Phys. B191, 217 (1981).

[57] A. Mitov and S.-O. Moch, Nucl. Phys. B751, 18 (2006), hep-ph/0604160.

[58] G. Altarelli, R. Ellis, G. Martinelli, and S.-Y. Pi, Nucl.Phys. B160, 301 (1979).

[59] M. Cacciari, P. Nason, and C. Oleari, JHEP 0604, 006 (2006), hep-ph/0510032.

[60] SLD, K. Abe et al., Phys. Rev. D65, 092006 (2002), hep-ex/0202031.

[61] DELPHI Collaboration, J. Abdallah et al., Eur.Phys.J. C46, 569 (2006), hep-ex/0603046.

[62] L3, P. Achard et al., Phys. Rept. 399, 71 (2004), hep-ex/0406049.

[63] R. Corke and T. Sjöstrand, JHEP 1103, 032 (2011), 1011.1759.

[64] T. Sjöstrand and P. Z. Skands, Eur.Phys.J. C39, 129 (2005), hep-ph/0408302.

[65] M. Cacciari and G. P. Salam, Phys.Lett. B641, 57 (2006), hep-ph/0512210. 